\title{
WestVirginiaUniversity
}

THE RESEARCH REPOSITORY @ WVU

Graduate Theses, Dissertations, and Problem Reports

1999

\section{Dynamic stability of an elliptical pendulum with unilateral simple supports}

Heins Naim Karam

West Virginia University

Follow this and additional works at: https://researchrepository.wvu.edu/etd

\section{Recommended Citation}

Karam, Heins Naim, "Dynamic stability of an elliptical pendulum with unilateral simple supports" (1999). Graduate Theses, Dissertations, and Problem Reports. 948.

https://researchrepository.wvu.edu/etd/948

This Thesis is protected by copyright and/or related rights. It has been brought to you by the The Research Repository @ WVU with permission from the rights-holder(s). You are free to use this Thesis in any way that is permitted by the copyright and related rights legislation that applies to your use. For other uses you must obtain permission from the rights-holder(s) directly, unless additional rights are indicated by a Creative Commons license in the record and/ or on the work itself. This Thesis has been accepted for inclusion in WVU Graduate Theses, Dissertations, and Problem Reports collection by an authorized administrator of The Research Repository @ WVU. For more information, please contact researchrepository@mail.wvu.edu. 


\title{
DYNAMIC STABILITY OF AN ELLIPTICAL PENDULUM WITH UNILATERAL SIMPLE SUPPORTS
}

\section{Heins N. Karam}

Thesis submitted to the faculty of the College of Engineering and Mineral Resources at West Virginia University in partial fulfillment of the requirements for the degree of

\author{
Master of Science \\ in \\ Mechanical Engineering \\ Victor H. Mucino, Ph.D., Chair \\ Kenneth Means, Ph.D. \\ Mridul Gautam, Ph.D. \\ Morgantown, West Virginia \\ 1999
}

Keywords: Elliptical Pendulum with Unilateral Support, Rollover of Elliptical Tanks. 
DEDICATED TO

MY

FAMILY 


\section{ACKNOWLEDGEMENTS}

The author wishes to express his sincere gratitude to his research advisor Dr. Victor H. Mucino for his continuous guidance and encouragement that led to the completion of this study. His is particularly appreciative of his enthusiasm and support in the preparation of this thesis. Appreciation is extended to Dr. Kenneth Means and Dr. Mridul Gautam, members of the advisory committee, for their valuable comments and suggestions. The author acknowledges the support provided by the Department of Mechanical and Aerospace Engineering for this work.

The author offers grateful thanks to Mohamed Salem for his help in the preparation of this work. The author wishes to extend his appreciation to all of his friends around the world and in particular Alejandro Kiriakidis.

Finally, though it is beyond the limit of expressing it with words, the author would like to take this opportunity to thank his parents, Naim A. Karam and Dalila H. Karam, his brothers and sisters with their families for their never-ending love, along with the moral, encouragement and financial support that they provided over the years. 


\title{
ABSTRACT \\ Dynamic Stability of an Elliptical Pendulum with Unilateral Simple Supports
}

\author{
By Heins N. Karam
}

The study of rollover stability of partially filled tanker trucks often requires the use of mechanical pendulums to substitute the sloshing dynamics of the fluid. Cylindrical tanks have been effectively characterized by simple pendulums combined with some fixed masses. Just recently, a WVU team proposed the use of an elliptical pendulum to substitute the sloshing dynamics in partially filled elliptical tankers.

In this research, a trammel mechanism is configured as an elliptical pendulum with a unilaterally supported base. That is, the supports on either side can lift off the ground to allow for rollover under certain critical lateral accelerations. Two generalized coordinates are considered, one representing the angle of swing of the pendulum and a second one representing the tilt angle of the base (lift off the ground). This degree of freedom (tilt angle) is active only if the lateral accelerations are greater than certain critical value, giving rise to the unilateral support restraints at the base of the mechanism.

The initial angle of swing that made the pendulum base liftoff was determined under zero lateral acceleration. The minimum horizontal lateral acceleration that caused the base to liftoff was also calculated when the pendulum was at rest (in the vertical position). The angular displacement, angular velocity and angular acceleration were calculated with respect to time, for systems with fixed or unilateral supports.

A mathematical model was developed to simulate the dynamic response of the elliptical pendulum with unilateral constraints. A mode of instability was found in which the pendulum swings producing liftoff but not rollover, as the base "bounces" back-and-forth, from a one support only base to a simple supported base. 


\section{TABLE OF CONTENTS}

TITLE PAGE .. $\mathrm{i}$

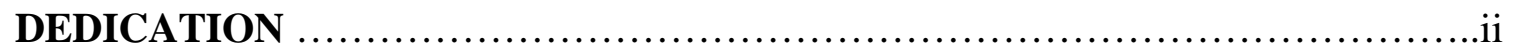

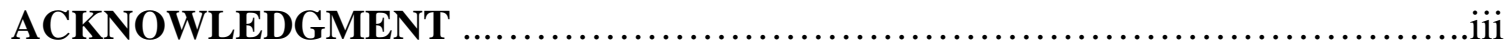

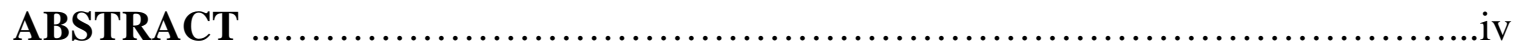

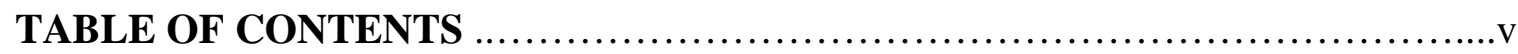

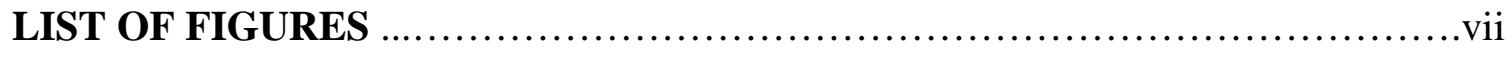

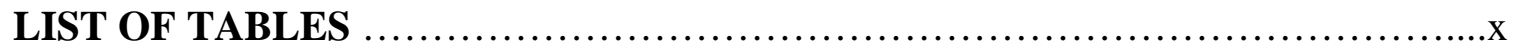

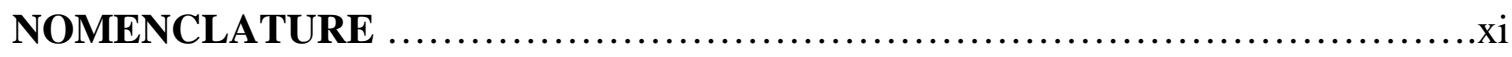

CHAPTER I. INTRODUCTION …..........................................

1.1 Introductory Remarks .............................................

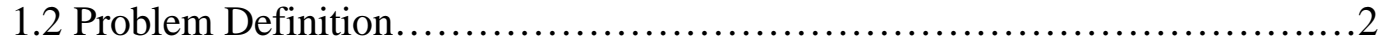

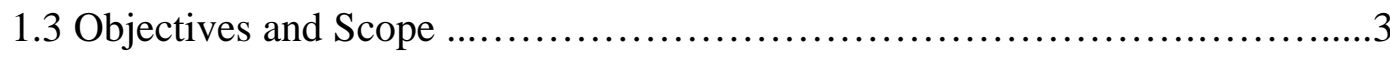

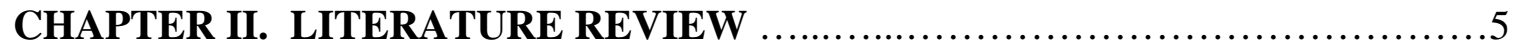

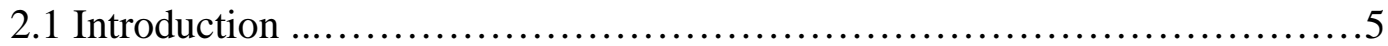

2.2 Previous Work Related to Vehicle Rollover ...............................6

2.3 Unilateral Constraints ...............................................14

CHAPTER III. PRELIMINARY ELLIPTICAL PENDULUM ANALYSIS............16

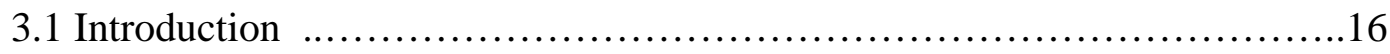

3.2 Development of the Elliptical Pendulum.................................. 16

3.3 Numerical Calculation for the Trammel Mechanism ........................19

3.4 Calculations for the Reactions at the Support..........................23

CHAPTER IV. SYSTEM WITH UNILATERAL SUPPORT ...................28

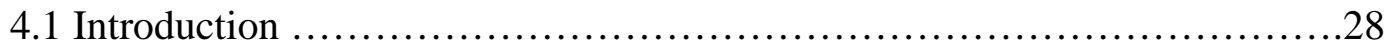

4.2 System during Stable Condition ........................................28

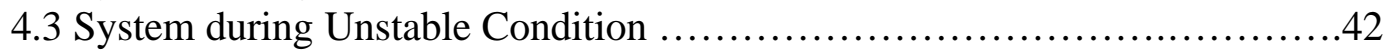

CHAPTER V. DISCUSSION OF RESULTS AND CONCLUSIONS …............45

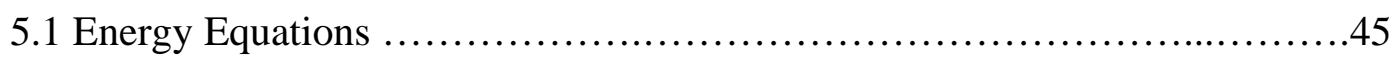

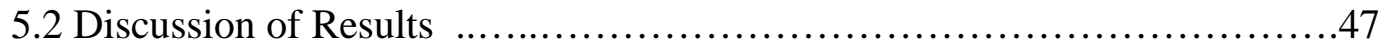

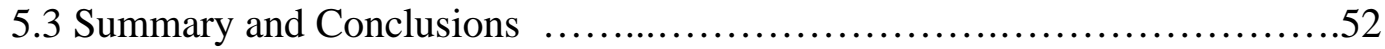


BIBLIOGRAPHY

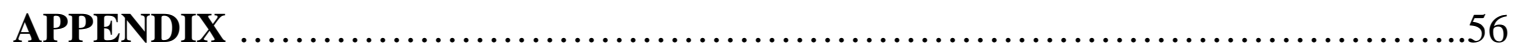

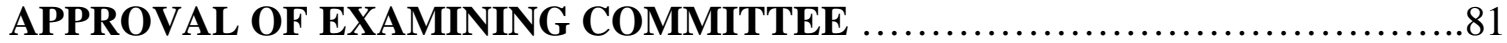




\section{LIST OF FIGURES}

Figure 2.1: The Different Cross-section of the Tanks Used in General Purpose

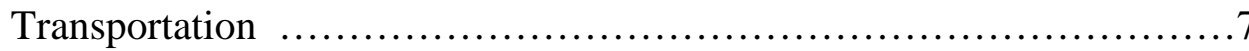

Figure 3.1: $\quad$ Steady State Motion of the Liquid Cargo under Vehicle Roll and Lateral Acceleration of an Elliptical Cross-section

Figure 3.2: The Location of the cg of a Given Volume of Fluid in an

Elliptical Tank

Figure 3.3: $\quad$ Path of cg of a Known Volume Drawn by an Elliptical

Trammel Mechanism

Figure 3.4: Angular Displacement vs Time for a Half-filled Tank .21

Figure 3.5: Angular Velocity vs Time for a Half-filled Tank .21

Figure 3.6: Angular Acceleration vs Time for a Half-filled Tank .22

Figure 3.7: $\quad \theta, \dot{\theta}$, and $\ddot{\theta}$ for an Elliptical Pendulum due to an External Horizontal Acceleration $\ddot{x}$

Figure 3.8: The Different Reactions that Act on a Partially Filled Tank with the Fluid Mass being simulated by a Trammel Elliptical Pendulum 24

Figure 3.9: Rolling Moment in the x-directions with respect to time .25

Figure 3.10: The Different Reactions at the Support in the Y-direction with respect to Time .26

Figure 3.11: The Two Reactions $\mathrm{R}_{1 \mathrm{y}}$ and $\mathrm{R}_{2 \mathrm{y}}$ with respect to Time .27

Figure 4.1: Unilateral Support System when is less than Zero (cw)...............29

Figure 4.2: $\quad$ Unilateral Support System when $\phi$ is greater than Zero (ccw).

Figure 4.3: Angles of Displacement with respect to Time during Unilateral Support

Figure 4.4: Both Angles Theta and Fi (in deg) at different Time when $\mathrm{R}_{1 \mathrm{y}}$ is Negative (measured cw). 
Figure 4.5: The Angular Velocity $\dot{\theta}$ with respect to Time when $\mathrm{R}_{1 \mathrm{y}}$ is Negative.......34

Figure 4.6: The Angular Acceleration $\ddot{\theta}$ with respect to Time

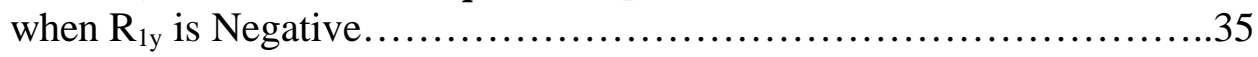

Figure 4.7: The Angular Velocity $\dot{\phi}$ with respect to Time when $\mathrm{R}_{1 \mathrm{y}}$ is Negative.......35

Figure 4.8: The Angular Acceleration $\ddot{\phi}$ with respect to Time when $\mathrm{R}_{1 \mathrm{y}}$ is Negative...........................................

Figure 4.9: Angles of Displacement with respect to Time during Unilateral Support............................................... 37

Figure 4.10: Both Angles Theta and Fi (in deg) at different Times

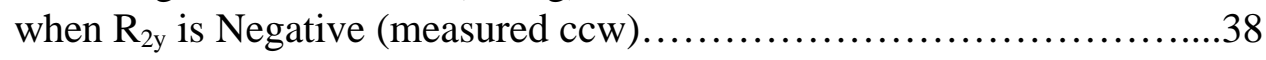

Figure 4.11: The Angular Velocity $\dot{\theta}$ with respect to Time when $R_{2 y}$ is Negative......39

Figure 4.12: The Angular Acceleration $\ddot{\theta}$ with respect to Time when $\mathrm{R}_{2 \mathrm{y}}$ is Negative..........................................

Figure 4.13: The Angular Velocity $\dot{\phi}$ with respect to when $\mathrm{R}_{2 \mathrm{y}}$ is Negative...........40

Figure 4.14: The Angular Acceleration $\ddot{\phi}$ with respect to Time when $R_{2 y}$ is Negative........................................40

Figure 4.15: Both Angular Displacements with respect to Time during One Complete Cycle.

Figure 4.16: Both Angles with respect to Time during Rollover...................42

Figure 4.17: Both Angles (in deg) when Rollover Occurs (measured cw)...........43

Figure 5.1: Kinetic Energy of the System with respect to Time ...................45

Figure 5.2: $\quad$ Potential Energy of the System with respect to Time $\ldots \ldots \ldots \ldots \ldots \ldots \ldots . \ldots 46$

Figure 5.3: Total Energy of the System with respect to Time $\ldots \ldots \ldots \ldots \ldots \ldots \ldots \ldots \ldots$

Figure 5.4: Critical Angle of Swing that will cause Liftoff but not Rollover at Different Lateral Acceleration (System is Initially at Rest). 
Figure 5.5: Critical Lateral Acceleration that will cause Liftoff but not Rollover (System is Initially at Rest) ........................................49

Figure 5.6: Critical Angle of Swing that will cause Liftoff but not Rollover (Zero Lateral Acceleration) ........................................50

Figure 5.7: Critical Angle of Swing for Pendulum with Zero Lateral Acceleration....51

Figure 5.8: Angle of Swing vs Horizontal Lateral Acceleration for System with initial Lateral Acceleration ...............................51 


\section{LIST OF TABLES}

Table 5.1: Critical Angles of Swing and Lateral Acceleration that will cause the System's Base to Liftoff (Under Different Initial Geometric Parameters) ..................................................48 


\section{NOMENCLATURE}

$$
\begin{aligned}
& \text { Re } \quad=\text { Reynolds Number } \\
& \alpha \quad=\text { Gradient of the Free Surface of Liquid } \\
& \theta_{S} \quad=\text { Sprung Mass Roll and Angle } \\
& a_{y} \quad=\text { Lateral Acceleration } \\
& Z_{t} \quad=\text { Lateral Coordinates of cg with respect to Tank-Chassis Structure cg } \\
& Y_{t} \quad=\text { Vertical Coordinates of } \mathrm{cg} \text { with respect to Tank-Chassis Structure cg } \\
& Z_{0} \quad=\text { Height of Liquid Cargo cg from the Tank Base under Static Condition } \\
& \mathrm{H}_{1} \quad=\text { Minor Axis of Tank } \\
& \mathrm{H}_{2} \quad=\text { Major Axis of Tank } \\
& \mathrm{m} \quad=\text { Mass of the Fluid inside the Tank } \\
& \text { a = Major Axis of the Elliptical Path } \\
& \mathrm{b} \quad=\text { Minor Axis of the Elliptical Path } \\
& \mathrm{h} \quad=\text { Distance between the Center of the Ellipse and the Supports in the y-direction } \\
& 1=\text { Half the Distance between the Supports } \\
& \theta=\text { Angular Displacement between Pendulum Arm and the Horizontal Axis } \\
& \dot{\theta}=\text { Angular Velocity between Pendulum Arm and the Horizontal Axis } \\
& \ddot{\theta} \quad=\text { Angular Acceleration between Pendulum Arm and the Horizontal Axis } \\
& x \quad=\text { External Horizontal Acceleration } \\
& \text { g = Gravity } \\
& \mathrm{R}_{1 \mathrm{x}} \quad=\text { Reactions at the Left Support in the } \mathrm{x} \text {-direction } \\
& \mathrm{R}_{1 \mathrm{y}} \quad=\text { Reactions at the Left Support in the } \mathrm{y} \text {-direction } \\
& \mathrm{R}_{2 \mathrm{y}} \quad=\text { Reactions at the Right Support in the y-direction } \\
& \phi \quad=\text { Angular Displacement between Tank and the Ground } \\
& \phi \quad=\text { Angular Velocity between Tank and Ground } \\
& \ddot{\phi} \quad=\text { Angular Acceleration between Tank and Ground } \\
& \mathrm{T} \quad=\text { Kinetic Energy } \\
& \mathrm{V} \quad=\text { Potential Energy }
\end{aligned}
$$




\section{CHAPTER I}

\section{INTRODUCTION}

\subsection{Introductory Remarks}

The rising interest of economics in freight transportation has prompted the trucking industries to examine the possible aspects of increasing the allowable size and weight limits for heavy vehicles. The trucking industry has carried out a number of studies with the collaborations of policy makers on the subject. Together they have established that the variations in the size and weight of heavy vehicles have resulted in significant changes in handling and maneuverability characteristics. This is especially true for tanker vehicles.

Tanker trucks are the prime movers of dangerous goods, chemicals and fuel oils on our highways. They encounter partial fill condition due to the varying weight density of the products, and the laws governing the axle loads, while those employed in fuel transportation encounter partial fill conditions during delivery routes $[1]^{\dagger}$. The cargo movement referred to as "sloshing" within the tank increases significantly with increase in the vehicle weights and dimensions. The handling and stability limits of the tanker trucks depend upon factors other than the normal trucking practices, such as: tank geometry; fill level; height of the center of gravity (cg); lateral and longitudinal load shift during turning, braking and lane change type highway maneuvers; and fluid-structure dynamic interactions [2].

A fluid in a road tanker experiences lateral excitation as a result of the continuous steering corrections made for the purpose of maintaining the general driving direction, under the influence of variable side winds, or simply through driving on a nonplanar road surface. 
This steering of a lane change maneuver will cause fluid sloshing and free oscillations. In addition, the braking and handling of the vehicle may cause longitudinal fluid motion resulting in severe impact of the fluid against the tank structure and forceful oscillations of the fluid thereafter, which may considerably endanger the safe handling of the vehicle [3].

\subsection{Problem Definition}

In studying tanker trucks, the interaction between fluid and vehicle structure is essential for establishing the response under lateral acceleration that may eventually cause rollover. To study this interaction in cylindrical tanker-trucks, certain mechanical analogies have been used in order to simplify the dynamic stability. These analogies include spring mass and simple pendulums.

The simple pendulum is not applicable for elliptical tankers since the center of gravity of the fluid inside a partially filled tank follows an elliptical path. Consequently, an elliptical pendulum is being used in the context of this thesis. An elliptical pendulum can be obtained by using a "trammel" mechanism in which the fluid mass is concentrated at the end of an arm with two sliders; one vertical and one horizontal (the mechanism can be seen in Figure 3.3).

In order to study stability, provisions must be made to allow the tanker to tilt as to liftoff one of the supports (presumably the onset of instability). In the trammel pendulum, the base support can only be in two possible states; flat or tilted (with one support lifted off the ground). The response of the system can be described by two degrees of freedom ( 2 d.o.f.) one representing the pendulum's swing angle and a second one representing the tilt angle.

\footnotetext{
${ }^{\dagger}$ Number (s) between square parenthesis designate reference (s) at the end of the thesis
} 
At zero tilt angle, only one degree of freedom is needed to produce the dynamic response. However, when the tilt angle is different from zero, the system results in a 2 d.o.f. system.

The challenge being addressed in the context of this thesis is to develop an approach to allow a system that switches between 1 d.o.f. to 2 d.o.f. (depending on the angle of lift) to be simulated. This is the nature of a "unilaterally supported structure," representing the liftoff of the supports. Of interest are the combinations of lateral acceleration and initial angle of swing that would make the pendulum base liftoff and if so, the combinations of acceleration and angle of swing that would make the base liftoff and not return, setting the threshold of instability.

\subsection{Objectives and Scope}

This research deals with stability of elliptical trammel mechanism under unilateral supports.. The main objectives of this study are as follows:

1. To conduct a literature review regarding the rollover of tank vehicles caused by the sloshing effect of the fluid at different percent of fill.

2. To study the dynamic behavior of an elliptical pendulum with unilateral supports simulating the fluid slosh inside an elliptical tank.

3. To find the initial angle of swing with zero lateral acceleration that would make the pendulum base liftoff.

4. To find the lateral acceleration that would make the base liftoff, with the pendulum initially at rest (angle of swing at $270 \mathrm{deg}$.). 
Matlab 5.2, user-friendly computer software was used for calculating and solving the stability equation of the elliptical pendulum. The results of the lagrangian equation were presented in the form of figures, as seen in the following Chapters to come. 


\section{CHAPTER II}

\section{LITERATURE REVIEW}

\subsection{Introduction}

Directional stability limits of partially filled liquid cargo vehicles are known to be significantly lower than those of the conventional rigid cargo vehicles due to the unique dynamic interactions between the vehicle and the sloshing liquid cargo. The forces and moments arising from a directional maneuver, yield considerable dynamic load shift in the roll and pitch planes due to sloshing of the liquid cargo within the partially filled tank. The dynamic load shift affects the directional stability of the partially filled tank truck in an adverse manner and can pose unreasonable risk to highway safety and the environment, when dangerous goods are hauled.

Fuel transportation in particular, requires large volume trailers, a condition that increases the center of gravity height, thus leading to poor vehicle handling and control [4]. An impressive number of studies [5] on fluid sloshing have been carried out since the sixties in different fields of applications: space vehicles, large ground tanks and canals, cargo ships, and road vehicles.

The linear theories of sloshing are based on the assumptions of potential flow, inviscid fluid, and linearized kinematics conditions at the free surface. These theories are in good agreement with the experiments, but only over a small range of motion amplitudes. The specifics of sloshing in road containers have forced the investigators to conduct separate studies rather than to apply readily available linear theories to the tanker design. However, the 
reported investigations are either experimental or also based on linear theory assumptions. The main drawback of the experimental investigations is that they are limited in range because of the high risk of experiments at limit performance. Because of these limitations, an approach based on numerical solution of the Navier-Stokes, continuity, and free-surface differential equations appears to be a good effective means for solving the sloshing problem [5].

\subsection{Previous Work Related to Vehicle Rollover}

Rakheja et al. [2] conducted a study on the most common tank cross-sections used in the transportation of chemicals and fuel oils. They computed the magnitude of the lateral and vertical translations of the center of mass of fluid from the roll plane model of a partially filled arbitrarily shaped tank. They also developed a computer program to simulate the steady turning characteristics of a four-compartment of the most common tank cross-sections used in transportation of chemicals and fuel oils. These different geometric shapes included circular,

elliptical, modified oval and modified square cross-sections as seen in Figure 2.1. The effect of the tank geometry on the rollover threshold was presented by comparing the tanks of various cross-sections when carrying the same payload and same fluid. They also presented the influence of lateral load shift within various tank cross-sections on the limited speed of tank vehicles. 


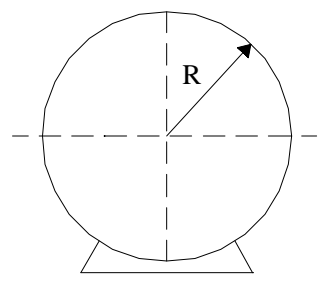

(a)

CIRCULAR

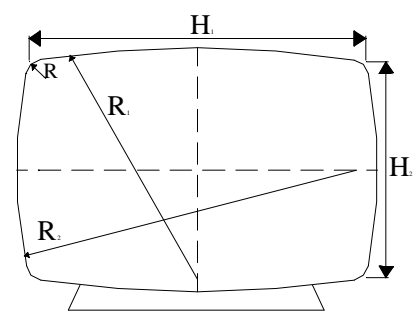

(c)

MODIFIED OVAL

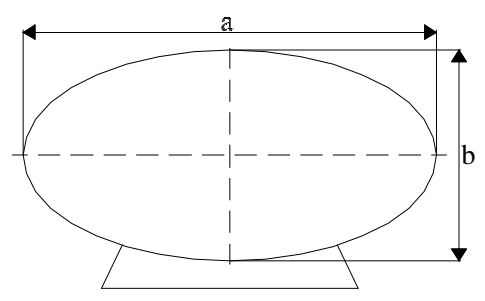

(b)

ELLIPTICAL

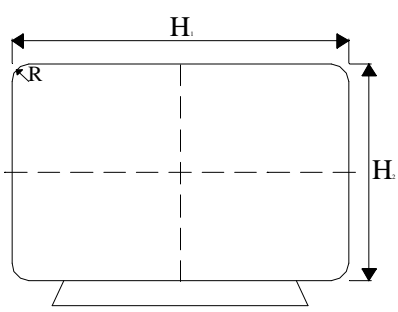

(d)

MODIFIED SQUARE

Figure 2.1: The Different Cross-section of the Tanks Used in General Purpose Transportation [2].

Their studies revealed that the magnitude of the lateral translation of the center of mass of fluid within a modified oval and modified square cross-sections, were much higher compared to that within circular and elliptical tanks especially for low fill levels. They also showed that the vehicles with modified oval and modified square tanks can rollover at lower values of lateral accelerations compared to the circular and elliptical cross-sections. However, the vertical translation of center of mass of fluid in circular and modified square configurations were significantly larger than that in a modified oval tank. In order to achieve the highest values of rollover acceleration limits, the researchers established an optimal order of compartment unloading and the location of the trailer composite axle [2].

Zhanqi et al. [4] through analysis of the vehicle model investigated the influence of number and sizes of compartments on the longitudinal load transfer, and the braking 
performance of a partially filled tank truck. They developed a kineto-static model of the fluid slosh within a partly-filled compartment ellipsoidal tank to determine the magnitude of dynamic load transfer as a function of the fill level, braking strength, tank geometry, and the location of partition walls. They also integrated the fluid slosh model to the vehicle model to derive the total tank vehicle model. Through a comparison of the analytical and experimental results, they showed quite similar trends in the response behavior with variations in the compartment sizes. Their test data correlated very well with the model results for all the compartment configurations. The simulation and test results both revealed that the longitudinal load transfer and thus the braking performance could be enhanced by optimally locating the partition walls, such that all compartments are of equal length. Through the results, they clearly demonstrated that selecting equal size compartments could minimize the stopping distance, braking time and time lag. The stopping distance, braking time and time lag performance, however, tended to increase considerably with large size partly filled tanks used in tractor-trailer combinations, due to the associated excessive load transfer.

The fluid movement within a partially filled tank truck, and its influence on the directional response characteristics, were investigated through a field test program [6]. This program which was undertaken jointly by the CONCAVE Research Center and Transportation Technology and the Energy Branch of Ontario Ministry of Transportation revealed that the magnitude of dynamic fluid slosh is strongly related to the vehicle speed, lateral and longitudinal acceleration, and the fill level. As a test vehicle, a semi-transparent scaled size tank was fabricated and mounted on a two-axle tractor. The tank assembly was located on the tractor such that the geometric center of the tank lies directly above the center of the rear axle 
in the roll plane. Water was selected as the cargo and a coloring agent was added to facilitate visualization of the fluid motion. Two video cameras were installed, one at the rear and the other at the side of the vehicle, to record the fluid motion in the roll as well as pitch planes. The field tests were conducted for various loading and fill conditions, directional maneuvers, and speed. The test maneuvers included: 30-meter constant radius turn; single and double lane changes; braking in a straight line; and braking in a turn. The fluid free surface in the roll plane was derived from the video records for various test maneuvers and compared to that established from the simulation software based on the steady-state fluid slosh analysis. The study revealed that the dynamic load transfer due to the fluid slosh could be accurately predicted by the steady-state fluid slosh analysis. The comparison between the field measured data analysis and the simulation software, clearly demonstrated that the software can be effectively used to investigate the directional stability of partially filled tank vehicles for constant speed directional maneuvers [6].

Assuming inviscid flow conditions, Ranganathan et al. [7] investigated the influence of the lateral load shift on the dynamic response characteristics of an articulated tank vehicle. A quasi-dynamic roll plane model of partially filled circular cross-section was developed and integrated to a three-dimensional model of an articulated vehicle. In their study to demonstrate the influence of dynamic fluid load shift, they compared the directional response characteristics of the tank vehicle computed for constant steer input to those of an equivalent rigid cargo vehicle. The comparative study revealed that the liquid load shift encountered during constant steer input would affect the directional stability of the tank vehicle considerably. Although the liquid load shift encountered during constant steer maneuver would influence the distribution 
of the cornering forces between the two tracks of the tank vehicle, leading to considerable deviation in the path followed by the tank vehicle. The researchers concluded that the directional response of the tank vehicle is strongly dependent on the fill condition, vehicle speed and the steer input. Their results showed that the destabilizing effects of the liquid load shift became quite apparent, when the fill level is increased to $70 \%$, due to the excessive load shift from the inner to the outer track. The directional response of the partially filled tank vehicle under constant axle loads was also investigated and compared to that of an equivalent rigid cargo to demonstrate the influence of large magnitude of destabilizing forces arising from the load shift on the directional stability of the vehicle. In their comparison of the roll and lateral acceleration response of the rigid cargo vehicles, they indicated the improved stability of the $40 \%$ filled vehicle when compared to a $70 \%$ filled vehicle, due to the low overall center of gravity of the $40 \%$ filled rigid cargo vehicle. They also concluded that at $70 \%$ filled tank vehicle with $4^{\circ}$ constant steer input, the vehicle would exhibit unstable behavior leading to rollover.

A simple methodology was proposed by Rakheja et. al [8] to estimate the rollover threshold of partially filled liquid cargo vehicle. The primary overturning moment caused by the lateral motion of the liquid cargo within the circular, elliptic, or modified-oval cross-section tanks was delivered as a function of the lateral acceleration, roll angle, fill level, and the tank geometry. The rollover threshold was estimated using two different methods. In the first method, the rollover was estimated by balancing the total overturning and restoring moments using a single degree-of-freedom (d.o.f.) model of the rigidly suspended tank vehicle. The roll moments arising from the multiple-axle suspension and tyres, individual axle loads, and lateral 
displacement were computed and integrated in the analysis in the second method. The results illustrated that the rollover threshold of partially filled circular tank vehicle could be accurately estimated using the proposed simplified approach. The rollover threshold of rigid cargo vehicles increased with a low fill level due to reduced cg height. However, the comprehensive static roll analysis revealed almost constant rollover threshold value of the liquid tank vehicle.

While the simplified approach [8] provided an accurate estimation of rollover threshold for circular cross-section tank vehicles, it yielded relatively larger errors for modified-oval tank vehicles, especially for low fill levels. This error was attributed to two primary assumptions: (i) the elliptic approximations utilized in computing the lateral coordinates of shifted cargo within a modified-oval tank; and (ii) negligible changes considered in the vertical coordinate of liquid cargo cg. For the elliptical tank, the simplified approach provides an accurate estimate of the rollover threshold. The rollover threshold limits estimated for constant and variable payload were compared to the values computed from the comprehensive kineto-static software to demonstrate the effectiveness of the simplified approach. In the case of the equivalent rigid cargo vehicles, the decrease in cg height due to reduced payloads (fill level) increased the rollover threshold limits of the vehicles. At the end, the researchers concluded that the threshold values computed using the two approaches tend to correlate very well at low fills in all cases except for the modified-oval tank vehicle [8].

Ying et al. [9] developed a novel approach to investigate the behavior of partially filled tank vehicles during straight-line braking maneuvers was developed by integrating an equivalent mechanical system model of the fluid to the vehicle model. Integrating the fluid slosh model of a partially filled tank to the pitch plane vehicle model developed the computer 
simulation model. They compared the braking characteristics of the tank vehicle incorporated with the equivalent spring-mass model to those obtained using a steady state fluid model. By approximating the tank through a number of thin rectangular sections, and then summing up the individual sections to obtain the overall system parameters, they developed the equivalent mechanical model for a cleanbore cylindrical tank used in transportation of bulk fluids. Later they investigated the dynamic behavior of fluid within tank vehicles during straight-line braking maneuvers. The equivalent mechanical system parameters representing the fluid slosh behavior within a cleanbore cylindrical tank were computed using the potential flow theory and were validated against experimental results. They found that the tank vehicle experienced dynamic load shift from the rear to the front when the vehicle was subject to a constant acceleration. Also in the analogy model of the spring-mass, the researchers indicated that the frequency of oscillation of the dynamic response depended on the fill level in the tank. The mean value of the normal load increased on the tractor front and rear axles, while the trailer axles experienced a decrease in the normal load. They concluded that the spring-mass model exhibited oscillatory response even after tank vehicle coming to a complete stop, representing the dynamics of the fluid motion within the partially filled tank.

Popov et al. [10] obtained the steady state and transient solutions in terms of amplitudes and damped natural frequencies of the main sloshing parameters for fluid in horizontal cylindrical road containers which were subjected to a step acceleration input, simulating a steady turn vehicle maneuver. The steady state solutions were derived analytically from the hydrostatic equations. The transient solutions were obtained by numerical integration of the Navier-Stokes, continuity, and free-surface equations. Popov et al. [10] proved that the 
same approach of replacing rotational motion by rectilinear motion could also be used for the transient response, since the error is of the same order as in the steady state. This was confirmed by rotational and rectilinear motions at identical conditions. In their study, they dictated three main parameters of the fluid motion: the Reynolds number; the magnitude of input acceleration, and the fill level. The Re number had very small effect on the magnitudes and frequencies of sloshing parameters in the range of $R e>10^{3}$. For $R e<10^{3}$ the difference in the magnitudes and frequencies rapidly increased in such a way that more viscous fluids vibrated more slowly, with smaller amplitude and with stronger decay. Each increase in the lateral acceleration increased the magnitude of the lateral force moment and decreased the slosh frequency. Another strong factor in influencing the sloshing loads was the fill level. The researchers showed that an increase in the fill level decreased the amplitude of slosh parameters and increased the slosh frequency. By revealing that linearized theory holds only for small amplitudes of fluid vibrations, they proved their prediction of fluid motion by a non-linear model for the case of intensive sloshing.

\subsection{Unilateral Constraints}

Many applications in industry involve mechanical systems interacting with the environment. An important task is to model the complete behavior so that the system and constraint are presented in a natural way. In most constrained mechanical systems, the nature of the constraint is best described by its unilateral behavior. The constraint, almost always, divides the workspace of a mechanical system into three regions. A region where the constraints are strictly satisfied and the mechanical system behaves freely, a region defining the boundary conditions of the constraint, and a region representing the violation of the constraint. 
Zefran and Kumar [11] showed how optimal control problems with inequality constraints could be effectively solved using simple numerical methods when they are reformulated as variation problem. They considered two key ideas, first the transform of inequalities into equality constraints with the help of slack variables, second the use of an integral form of the necessary conditions. This made the method transparent to the switching between different constraint manifolds. This approach offered a general method to obtain globally optimal solutions for the force distribution as well as for the joint trajectories.

Another study dealing with the behavior of robots with unilateral constraint was made by Pfeiffer [12]. In his study, he revealed that the equations of motion are accompanied by inequality constraints due to unilateral contacts, and that the whole system of equations is put into a complementarily form. This follows the basic rule of contact dynamics stating that for each contact either relative kinematics will be zero and then the relevant constraint forces will not be zero or vice versa. Therefore the product of magnitudes of both groups is always zero. Furthermore, a contact going from a passive to an active state is indicated always by magnitudes of relative kinematics. Constraint forces (normal or tangential forces) always indicate an active (stick) contact going to be passive (slip). The evaluation of relative kinematics is strongly connected with a parameterization of the surface contours and application of differential geometry.

Wosle and Pfeiffer [13] showed that using the Lagrange multiplier methods with a mathematical formulation of the contact problem could solve large systems with many constraints very efficiently. The differential-algebraic equations of a system with dependent 
constraints and planar friction were also considered, and an iterative algorithm was presented for the evaluation of such problem. Their research was based on transformations of the kinematic secondary conditions in the form of inequalities to equations. Ultimately, they end up with a solvable nonlinear system of equations consisting of the differential equations of motion, the constraint equations and the projections of the constraint forces. 


\section{CHAPTER III}

\section{PRELIMINARY ELLIPTICAL PENDULUM ANALYSIS}

\subsection{Introduction}

During delivery routes, the fluid in a road tanker experiences lateral excitation as a result of the continuous steering corrections. This steering maneuver will cause the fluid to slosh inside the partially filled tank. This chapter deals with the construction of an elliptical pendulum that will simulate the path of the center of gravity (cg) of fluid inside a partially filled elliptical tank. Section 3.2 describes the method used to demonstrate that the cg of the fluid mass inside an elliptical tank will follow an elliptical path proportional to the elliptical dimensions of the tank [15]. Section 3.3 includes the calculations of different angles of displacement, velocity, and acceleration for the elliptical trammel pendulum. Section 3.4 includes the calculations for different reactions at the support of the tank.

\subsection{Development of the Elliptical Pendulum}

Previous literature has shown that the first lateral mode of sloshing in a container due to any lateral excitation is an anti-symmetric mode, and that it is the dominant mode. It was also determined that the angular movement of the straight line of the surface of the fluid (neglecting longitudinal sloshing) can present this anti-symmetric dominant mode [15].

Rakheja [8] studied the location of the center of gravity for a given fluid in an elliptical tank assuming the surface motion above. This study determined that the center of gravity of any fluid bulk translates along a concentric ellipse for a given fill level, lateral acceleration and 
sprung mass roll angle. Figure 3.1, shows the geometry of a partially filled elliptical tank and the path followed by the cg of the fluid volume at certain roll angle.

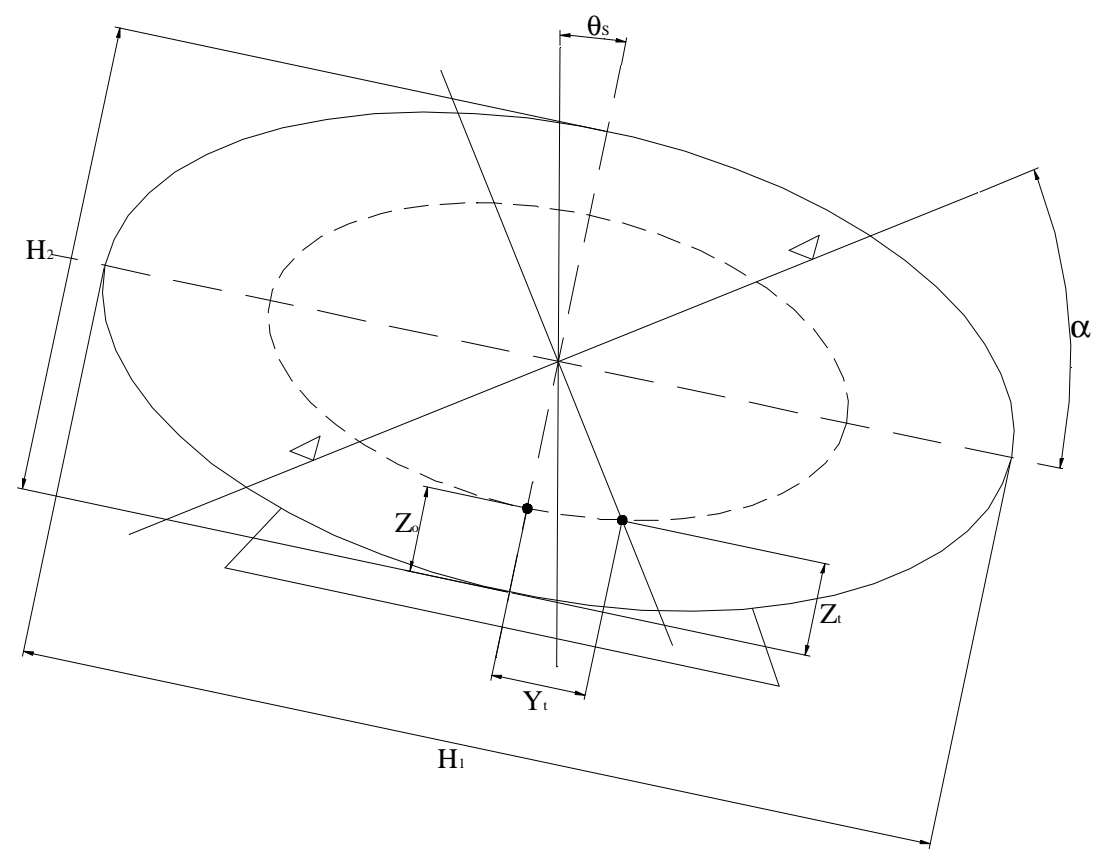

Figure 3.1: Steady State Motion of the Liquid Cargo under Vehicle Roll and Lateral Acceleration of an Elliptical Cross-section [8].

By assuming small angles, Rakheja [8] expressed the gradient of the free surface of fluid $(\alpha)$ as a function of sprung mass roll and angle $\left(\theta_{S}\right)$ and lateral acceleration $\left(a_{Y}\right)$ :

$$
\alpha=\frac{a_{y}+\theta_{S}}{1-a_{y} \theta_{s}}
$$

where $a_{y}$ is in $\mathrm{g}$ units and $\theta_{S}$ is in radians. The vertical and lateral coordinates of $\mathrm{cg}$ of an elliptical cross-section tank were derived form the geometry, and were expressed as:

$$
Z_{l}=0.5 H_{2}(1-\cos \alpha)+Z_{0} \cos \alpha
$$




$$
Y_{l}=\left[0.5-\frac{Z_{0}}{H_{2}}\right] H_{1} \sin \alpha
$$

where $Z_{l}$ and $Y_{l}$ are the lateral and vertical coordinates of the cg of fluid with respect to the tank-chassis structure cg, respectively. $Z_{0}$ is the cg height of fluid cargo with respect to the tank base under static condition. $\mathrm{H}_{2}$ and $\mathrm{H}_{1}$ are the major and minor axes, respectively. By knowing the cg location of a partially filled elliptical tank, we automatically know the minor axis of the elliptical shape that the cg will follow. Figure 3.2 shows the path of the cg for an arbitrary partially filled elliptical tank. This path falls on a contour parallel to the tank wall such that $\frac{a}{b}=\frac{A}{B}[15]$.

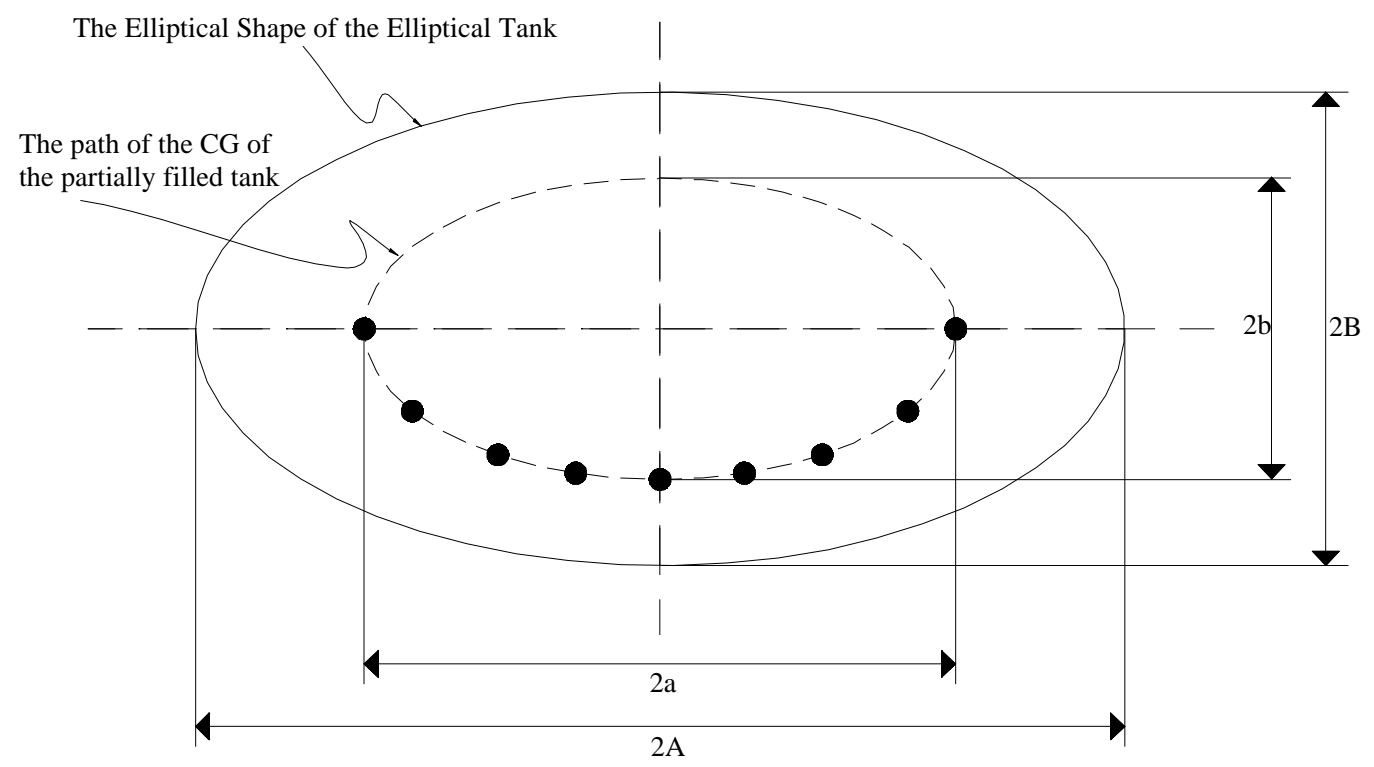

Figure 3.2: The Location of the cg of a Given Volume of Fluid in an Elliptical Tank [15].

Thus, a pendulum that follows an elliptical path would simulate the dynamic effect of the first mode of the fluid sloshing. 


\subsection{Numerical Calculation for the Trammel Mechanism}

The elliptical trammel mechanism was an idea inspired by Leonardo DeVinci in the $16^{\text {th }}$ century, which he used to trace an elliptical path on paper [16]. It consists of three points one sliding vertically, one sliding horizontally and one being the tracer. The mechanism is shown in Figure 3.3. A dynamic study of this pendulum has been studied thoroughly to obtain the values of its parameters ( $\mathrm{m}, \mathrm{a}$, and $\mathrm{b}$ ) [15]. With $\mathrm{m}$ being the mass of fluid in the tank, a and $\mathrm{b}$ being the major and minor axes of the elliptical path of this mass, respectively. Figure 3.3 also shows the important notation used in deriving the equation of motion of the pendulum and finding the forces exerted due to its motion.

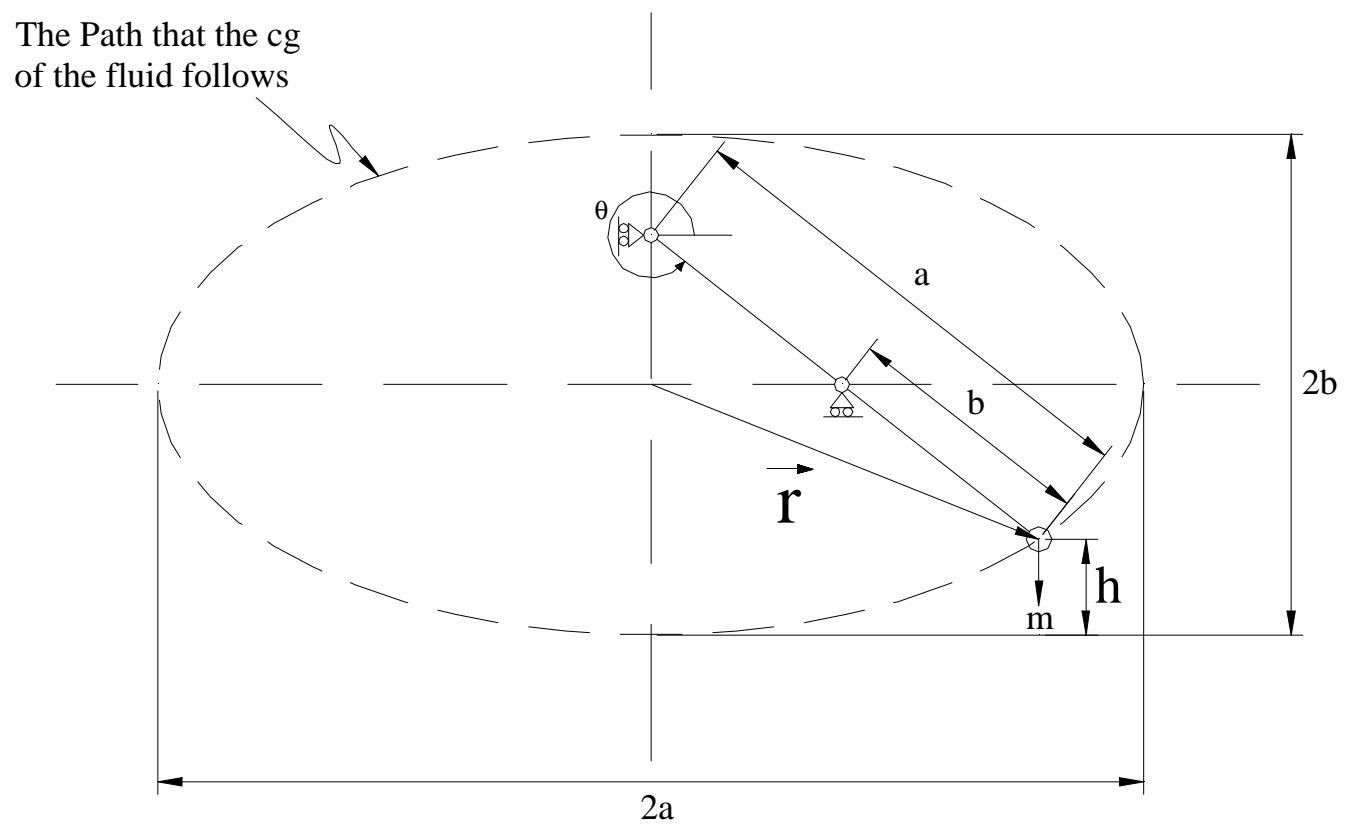

Figure 3.3: Path of cg of a Known Volume Drawn by an Elliptical Trammel Mechanism

When the tank is at rest, the pendulum arm was found to be perpendicular to the fluid surface, the angle $\theta$ (angle between the pendulum arm and the horizontal axis) was taken as 
the parameter used in deriving the equation of motion of the pendulum. Lagrangian method was used to derive the equation of motion for a fixed support (see details in the Appendix) and was found to be:

$$
\ddot{\theta}\left(a^{2} \sin ^{2} \theta+b^{2} \cos ^{2} \theta\right)+1 / 2 \dot{\theta}^{2}\left(a^{2}-b^{2}\right) \sin (2 \theta)+g b \cos \theta=0
$$

where $\theta$ is the angular displacement, $\dot{\theta}$ is the angular velocity, and $\ddot{\theta}$ is the angular acceleration of the system. The above equation is a nonlinear second order differential equation, and could be written as a system of coupled first order differential equation as follows:

$$
\begin{aligned}
& \dot{\theta}=x_{2} \\
& \ddot{\theta}=\frac{1 / 2 * x_{2}{ }^{2} *\left(b^{2}-a^{2}\right) \sin \left(2 * x_{1}\right)-g b \cos \left(x_{1}\right)}{\left(a^{2} * \sin ^{2}\left(x_{1}\right)+b^{2} * \cos ^{2}\left(x_{1}\right)\right)}
\end{aligned}
$$

Equations 3-5, and 3-6 can be solved numerically to find the values of $\theta$, and $\dot{\theta}$ with respect to time, then by differentiating the graph for $\dot{\theta}$ with respect to time; we could find the values for $\ddot{\theta}$. Programming the last two equations into Matlab 5.2 (M-file can be seen in the Appendix) and inputting the time integral and initial condition for the angular displacement, should be enough to find the values of the three unknowns with respect to time. The angular displacement, angular velocity, and angular acceleration plots for a half-filled tank $(\mathrm{a}=$ $50.93 \mathrm{~cm}$, and $\mathrm{b}=25.87 \mathrm{~cm}$ ) with respect to time can be seen in Figure 3.4 through Figure 3.6, respectively. 


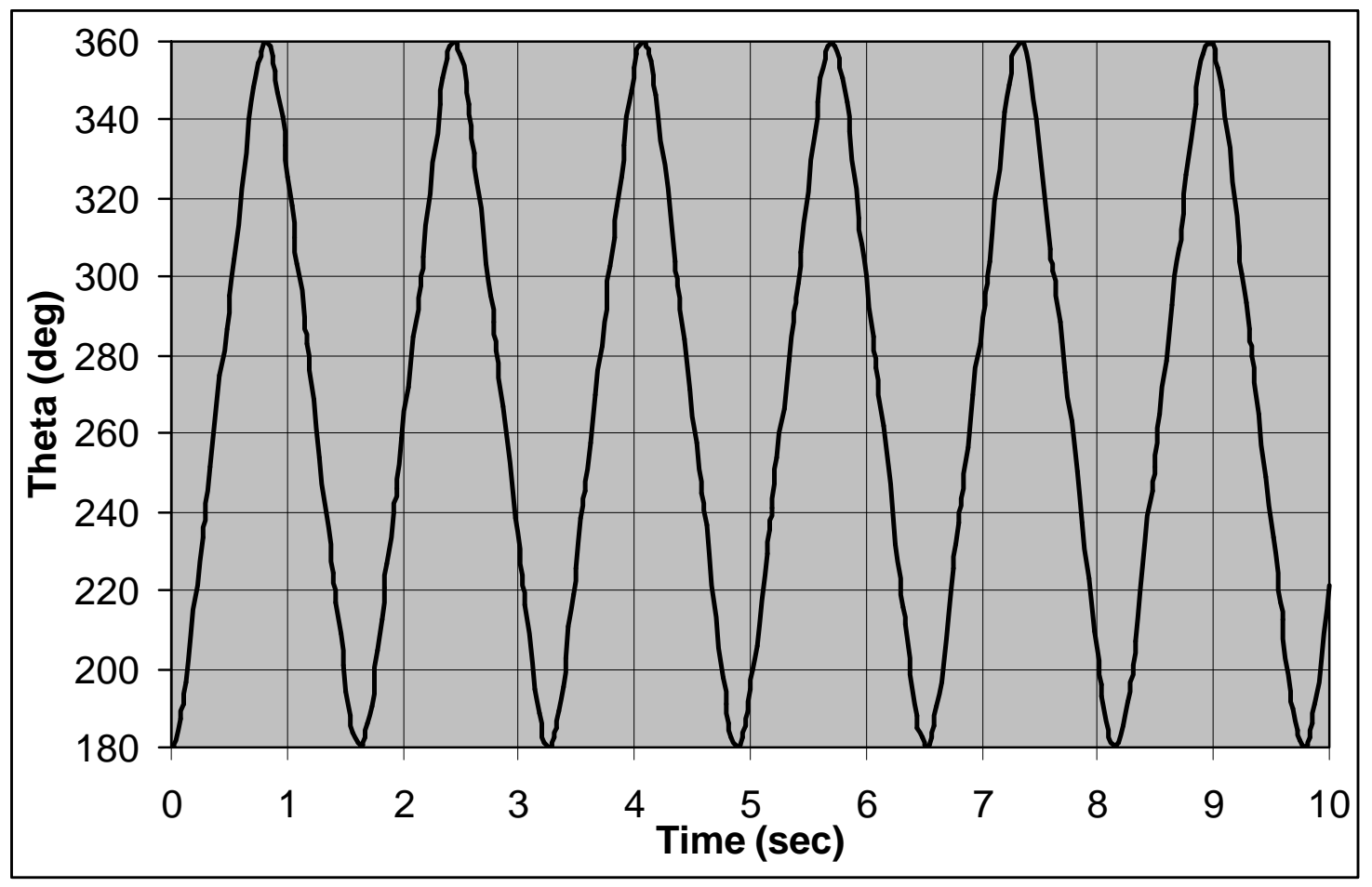

Figure 3.4: Angular Displacement vs Time for a Half-filled Tank

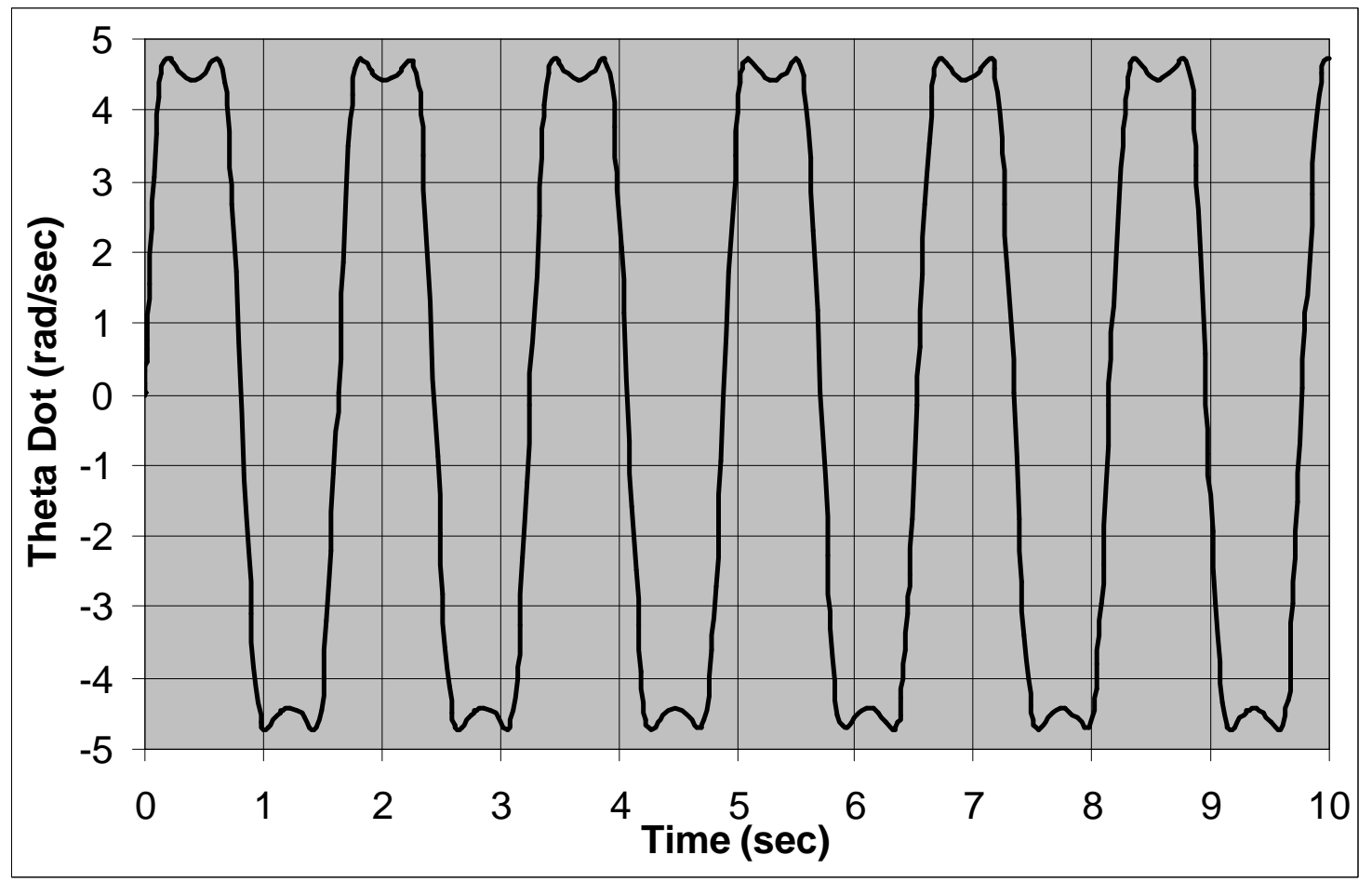

Figure 3.5: Angular Velocity vs Time for a Half-filled Tank. 


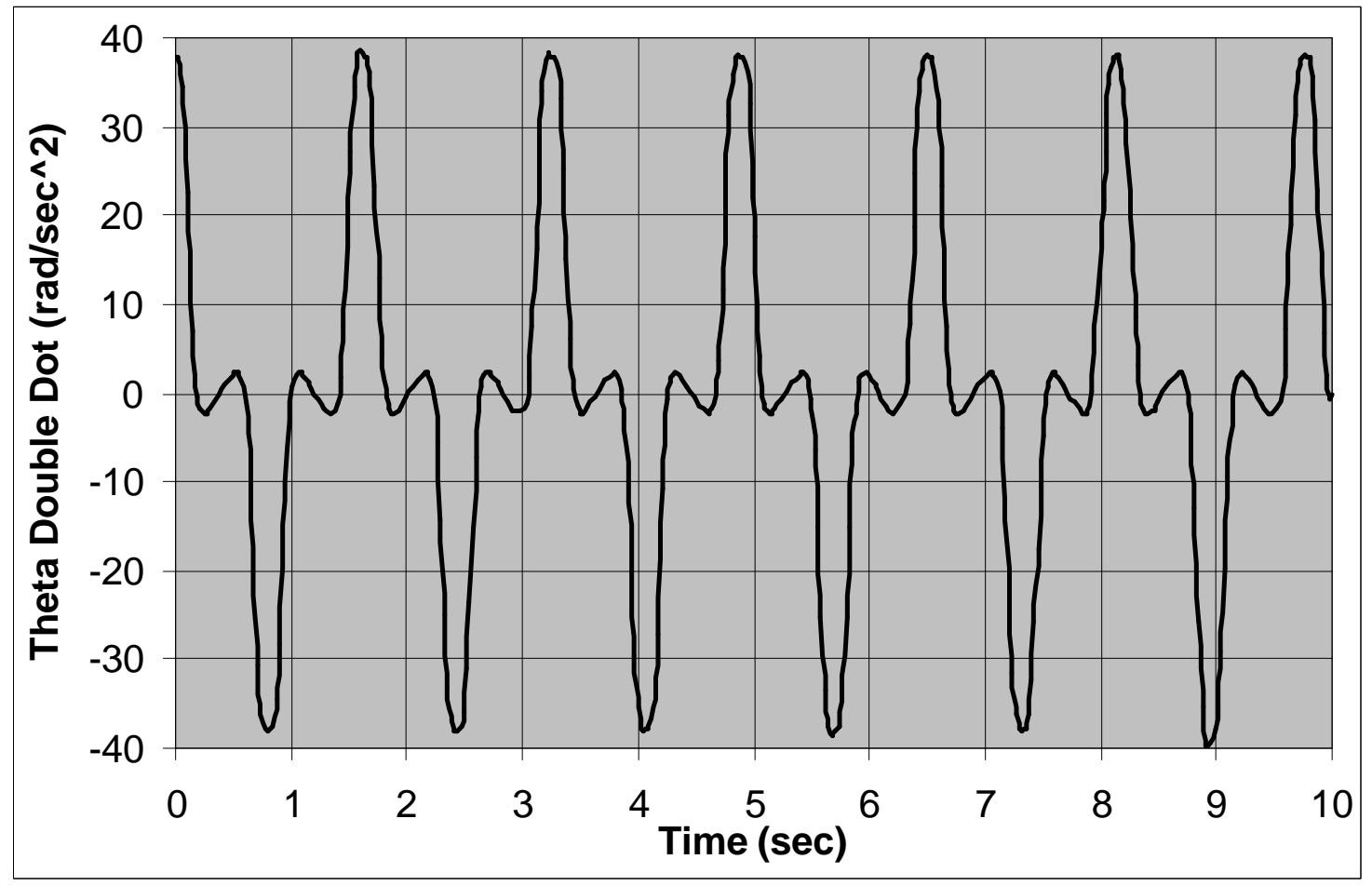

Figure 3.6: Angular Acceleration vs Time for a Half-filled Tank.

By allowing the tank to move in the horizontal $\mathrm{x}$-direction, the equation of motion for the elliptical pendulum due to the external horizontal acceleration $\stackrel{\bullet}{x}$ became:

$$
\ddot{\theta}\left(a^{2} \sin ^{2} \theta+b^{2} \cos ^{2} \theta\right)+1 / 2 \dot{\theta}^{2}\left(a^{2}-b^{2}\right) \sin (2 \theta)+g b \cos \theta-a \ddot{x} \sin \theta=0
$$

this equation is also a second order differential equation. By assigning $\ddot{x}$ the numerical value of $0.1 \mathrm{~g}$ (and without changing the values of a and b), Figure 3.7 shows the values for the angular displacement $\theta$, the angular velocity $\dot{\theta}$, and the angular acceleration $\ddot{\theta}$ with respect to time. 

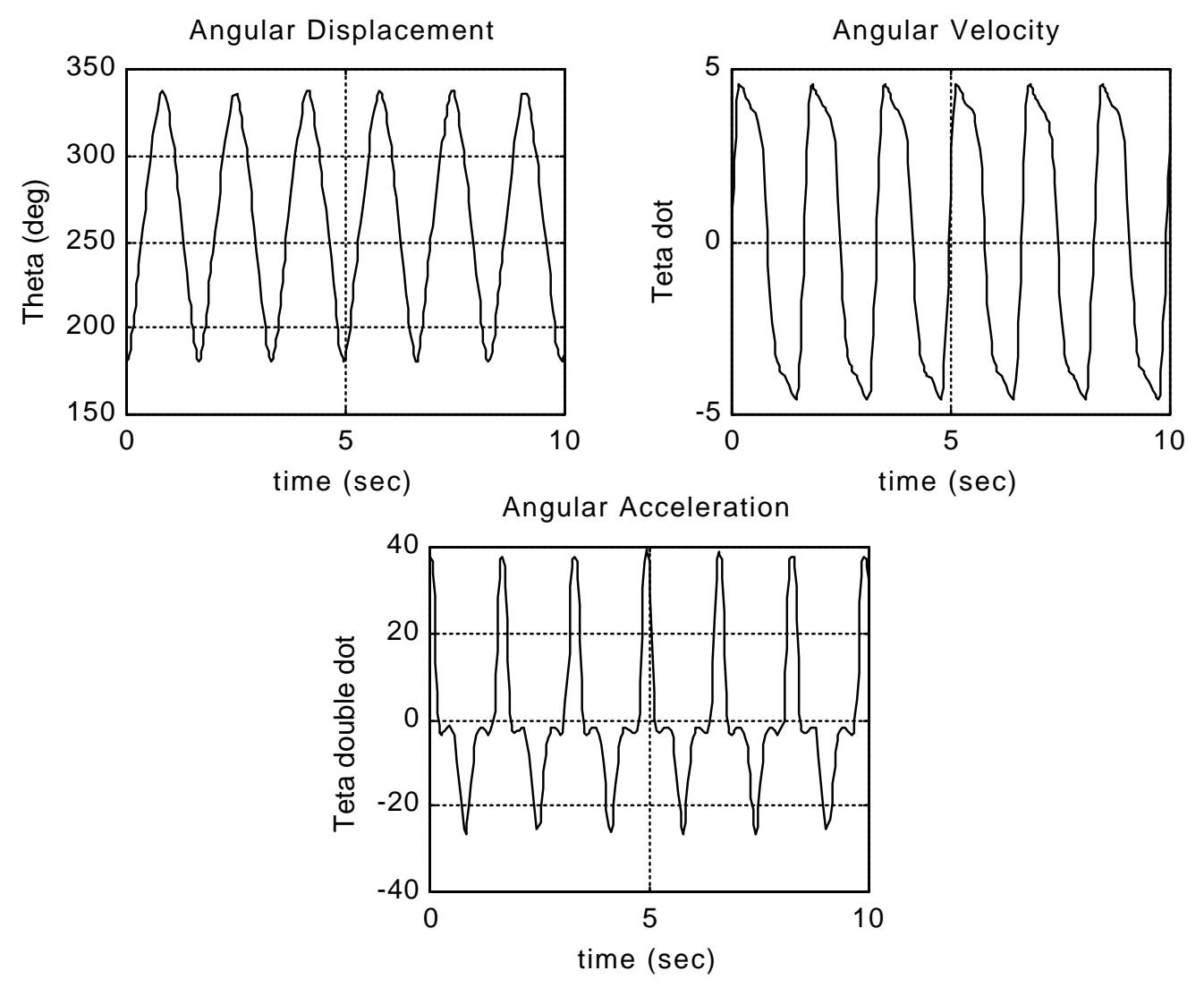

Figure 3.7: $\theta, \dot{\theta}$, and $\ddot{\theta}$ for an Elliptical Pendulum due to an External Horizontal Acceleration $\ddot{x}$

By comparing Figure 3.7 to Figures 3.4 through 3.6, we found that the values of $\theta, \dot{\theta}$, and $\ddot{\theta}$, went through a minor change for the elliptical pendulum with small horizontal acceleration, when compared to the values of the elliptical pendulum with fixed supports.

\subsection{Calculations for the Reactions at the Support}

In order to determine the stability of the elliptical pendulum, a study was carried out to calculate the reactions at the support. Figure 3.8 shows the pendulum arm at an arbitrary angle $\theta$. 


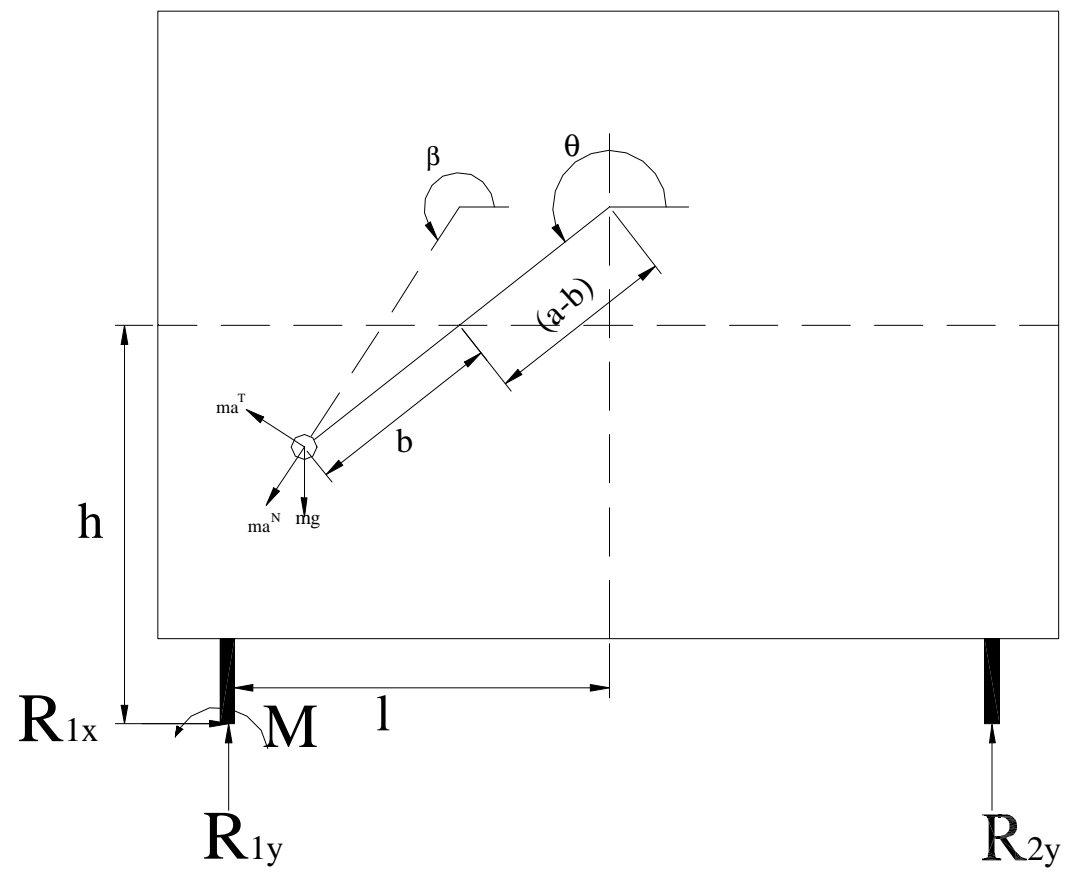

Figure 3.8: The Different Reactions that Act on a Partially Filled Tank with the Fluid Mass being simulated by a Trammel Elliptical Pendulum

Where 1 is half the distance between the two supports, $\mathrm{h}$ is the distance between the center of the ellipse and the ground, $\mathrm{a}$ and $\mathrm{b}$ are the major and minor axes of the elliptical path that the cg makes at a certain percent fill. Solving the free body diagram (FBD) of the above system yields the following equations:

$$
\begin{aligned}
& R_{1 x}=-m\left[b \dot{\theta}^{2} \cos \theta-\ddot{\theta} \sin \theta\right] \\
& R_{1 y}+R_{2 y}=m\left[g-a \dot{\theta}^{2} \sin \theta-\ddot{b} \cos \theta\right] \\
& R_{2 y}=\frac{m\left[(l+a \cos \theta)\left(g-a \dot{\theta}^{2} \sin \theta-b \ddot{\theta} \cos \theta\right)+(h+b \sin \theta)\left(b \dot{\theta}^{2} \cos \theta-a \ddot{\theta} \sin \theta\right)\right]}{2 l}
\end{aligned}
$$


The following three equations have three unknown $\left(R_{1 x}, R_{1 y}\right.$, and $\left.R_{2 y}\right)$ and could be solved numerically by assigning $\theta, \dot{\theta}$, and $\ddot{\theta}$ the values calculated in section 3.3 with the same percentage of fill (half-filled tank $a=50.93 \mathrm{~cm}$, and $b=25.87 \mathrm{~cm}$ ). Using Matlab 5.2 (see M-file in the Appendix) will yield the following plots:

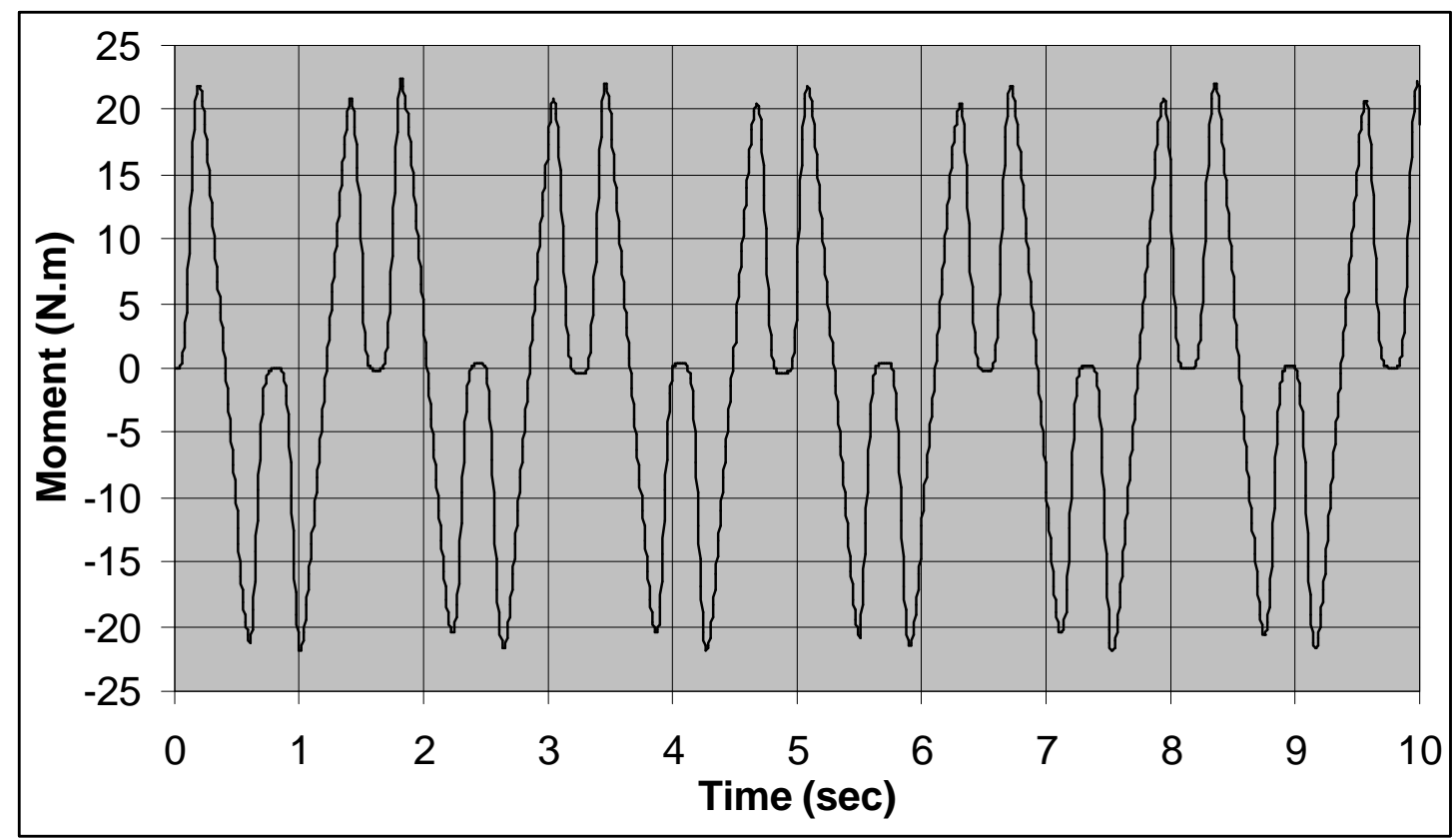

Figure 3.9: Rolling Moment in the x-directions with respect to time. 

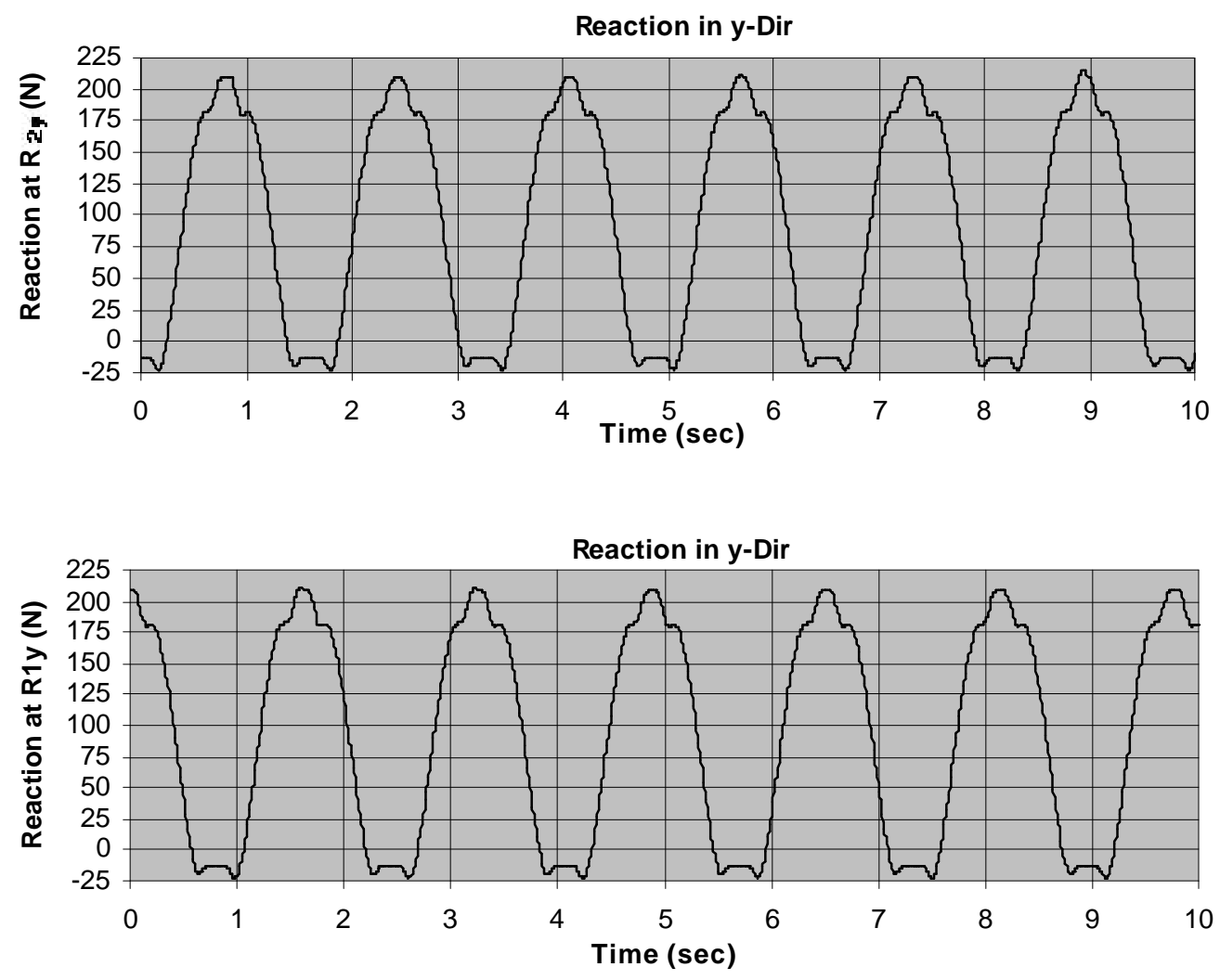

Figure 3.10: The Different Reactions at the Support in the $y$-direction with respect to Time

The above figure shows the reactions in the y-direction changing according to the angle of swing $\theta$. Comparing Figure 3.10 with Figure 3.4 shows that for $\pi<\theta<3 \pi / 2$ (the angle of swing being in the third quadrant) the reaction of $R_{2 y}$ is smaller than the reaction of $R_{1 y}$, both reactions are equal to each other when $\theta=3 \pi / 2$, and $R_{2 y}$ is greater than $R_{1 y}$ for $3 \pi / 2<\theta<2 \pi$ (fourth quadrant) which is exactly what we hopped for. 


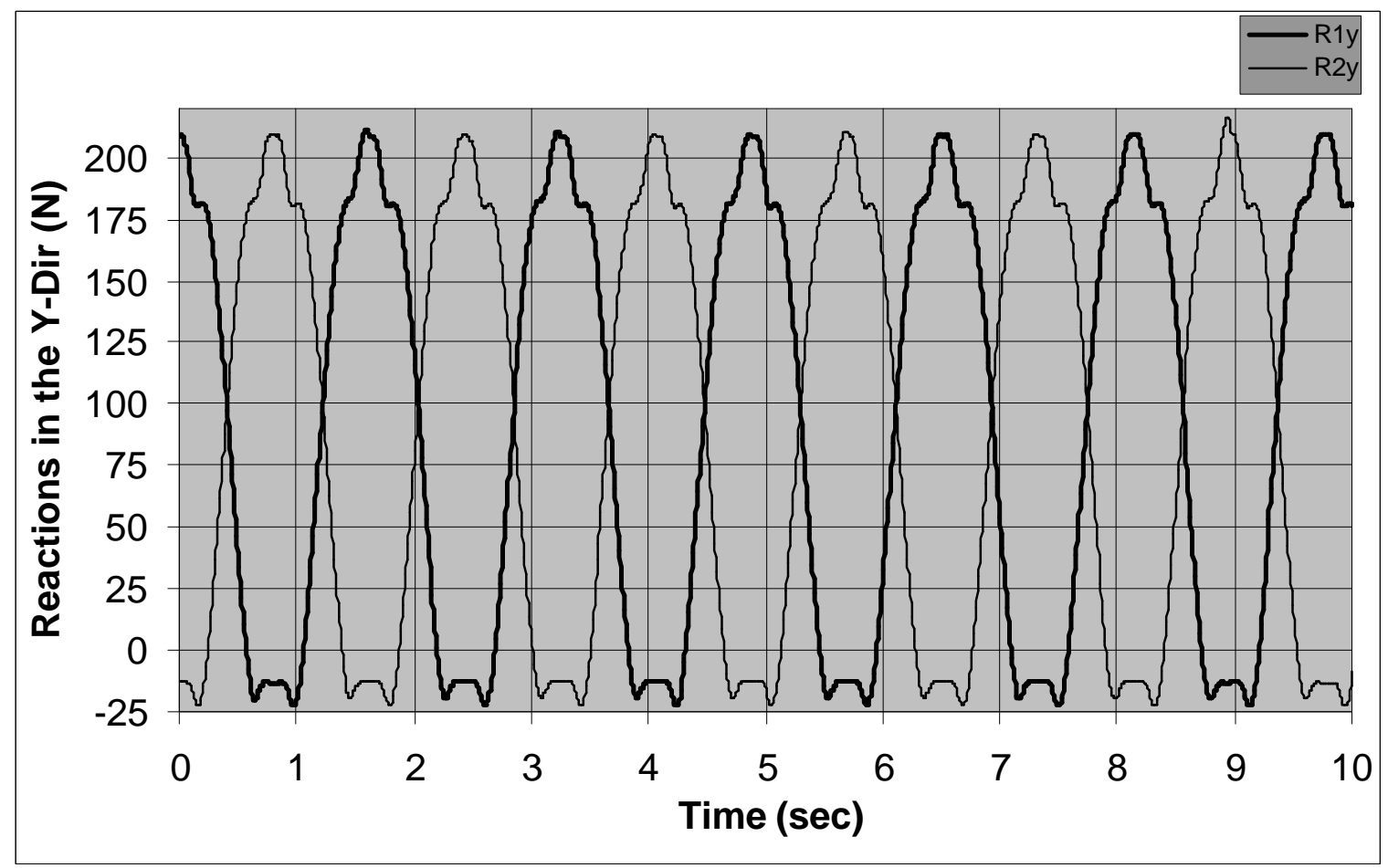

Figure 3.11: The Two Reactions $\mathrm{R}_{1 \mathrm{y}}$ and $\mathrm{R}_{2 \mathrm{y}}$ with respect to time.

By studying Figure 3.11 carefully, we conclude that the system shown in Figure 3.8 goes through unilateral support. When one of the reactions $\mathrm{R}_{1 \mathrm{y}}$ or $\mathrm{R}_{2 \mathrm{y}}$ becomes negative, the system (in this case the tank) will loose its support at that side, causing that support to liftoff of the ground. A more detailed and narrow study was made to find the angle of lift (angle that the tank's horizontal centerline makes with the ground) and is giving in chapter 4. 


\section{CHAPTER IV}

\section{SYSTEM WITH UNILATERAL SUPPORT}

\subsection{Introduction}

When the system is stable it is subjected to a single degree of freedom $\theta$, but when one of its supports loses contact with the ground, it becomes a two degrees of freedom system having $\theta$ and $\phi$ as the two unknowns. The new variable Phi $(\phi)$ is the angle of lift (angle of tilt) that the tank makes with the ground. Having a unilateral support will cause the equation of motion to change, which will require the derivation of new sets of equations and constraints for the Lagrange equation.

As will be seen later on in this chapter, the system could have one support and still be referred to as stable system. If the tank rocks back and forth from one support to the other before coming to a complete stop on both supports, it is known to be a stable system. If it rolls to one of its sides, then it is known to be an unstable system. Section 4.2 deals with a stable system under unilateral constraint with known initial conditions, while section 4.3 deals with an unstable system.

\subsection{System during Stable Condition}

In the previous chapter, we suspected that the system shown in Figure 3.8 could be unstable since the reactions in the $y$-direction $\left(R_{1 y}\right.$ or $\left.R_{2 y}\right)$ fluctuated one at a time between negative and positive values. In this chapter, the angle of swing $\theta$ and the angle of lift $\phi$ were 
calculated, along with their angular velocities and accelerations when the tank is subjected to a known horizontal acceleration $\ddot{x}$.

The moment the system becomes subjected to a unilateral support, it becomes a two degrees of freedom system, with $\theta$ and $\phi$ as the unknown. This will lead to two sets of second order differential equations that could be solved simultaneously.

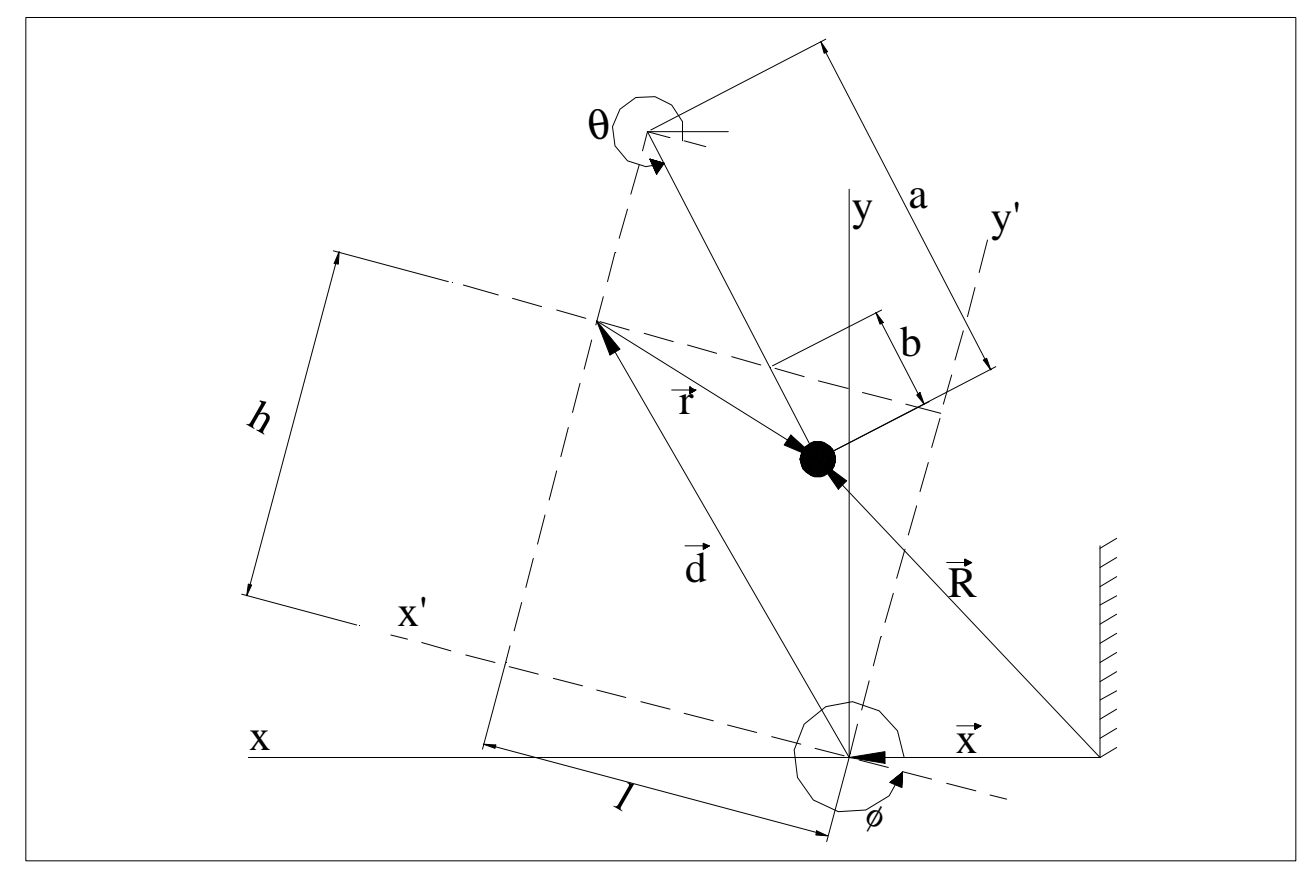

Figure 4.1: Unilateral Support System when $\phi$ is less than Zero (cw).

$$
\begin{aligned}
& \ddot{\theta}\left(a^{2} \sin ^{2} \theta+b^{2} \cos ^{2} \theta\right)+1 / 2 \dot{\theta}^{2}\left(a^{2}-b^{2}\right) \sin 2 \theta+\ddot{\phi}(a h \sin \theta-b l \cos \theta+a b) \\
& +\dot{\phi}^{2}\left[a l \sin \theta-b h \cos \theta-\frac{1}{2}\left(a^{2}+b^{2}\right) \sin 2 \theta\right]+g(b \cos \theta \cos \phi-a \sin \theta \sin \phi) \\
& +\ddot{x}(a \sin \theta \cos \phi+b \cos \theta \sin \phi)=0 \\
& \ddot{\theta}(a h \sin \theta-b l \cos \theta+a b)+\dot{\theta}^{2}(a h \cos \theta+b l \sin \theta)+\ddot{\phi}\left[(l-a \cos \theta)^{2}+(h+b \sin \theta)^{2}\right] \\
& +\ddot{\theta} \dot{\phi}\left[-2 a l \sin \theta+2 b h \cos \theta+\left(a^{2}+b^{2}\right) \sin 2 \theta\right]-g[(l-a \cos \theta) \cos \phi+(h+b \sin \theta) \sin \phi] \\
& -\ddot{x}[(l-a \cos \theta) \sin \phi-(h+b \sin \theta) \cos \phi]=0
\end{aligned}
$$


Equations 4-1a, and 4-1b are the two second order differential equations of motion for the system shown in Figure 4.1 under unilateral support having only $\mathrm{R}_{2 \mathrm{y}}$ (right side) touching the ground, a more detailed derivation of both equations could be seen in the Appendix. These two equations could be broken down into four equations of first order, with:

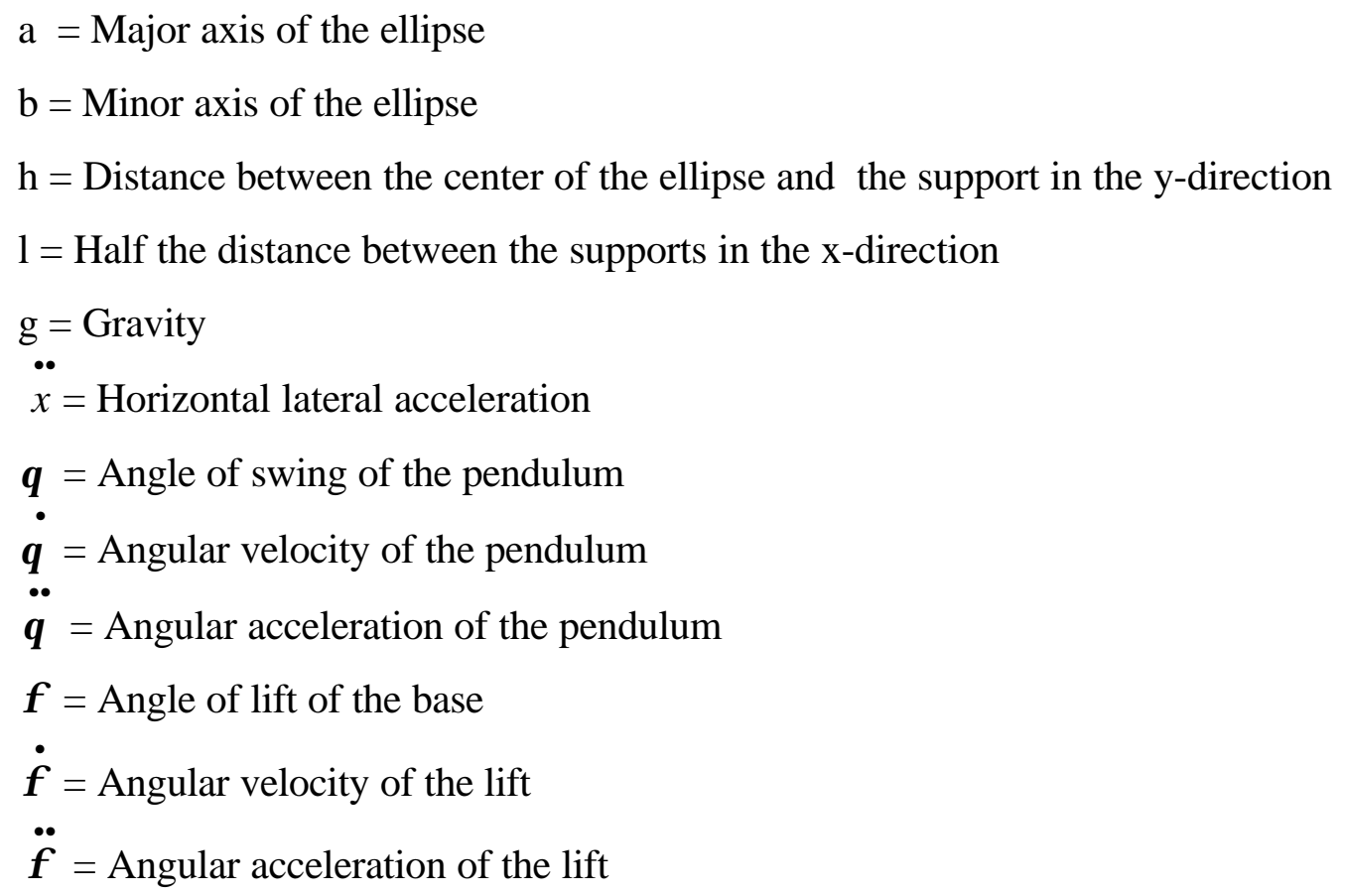

In order to use the Equations 4-1a and 4-1b, $\mathrm{R}_{1 \mathrm{y}}$ have to be negative which means that the angle of lift $\phi$ can only be less than zero (measured clockwise from the horizontal axis). Once the value of $\phi$ returns to zero, Equation 3-9 is to be used until the reaction $R_{2 y}$ becomes negative, at this time we are to use the following equations: 


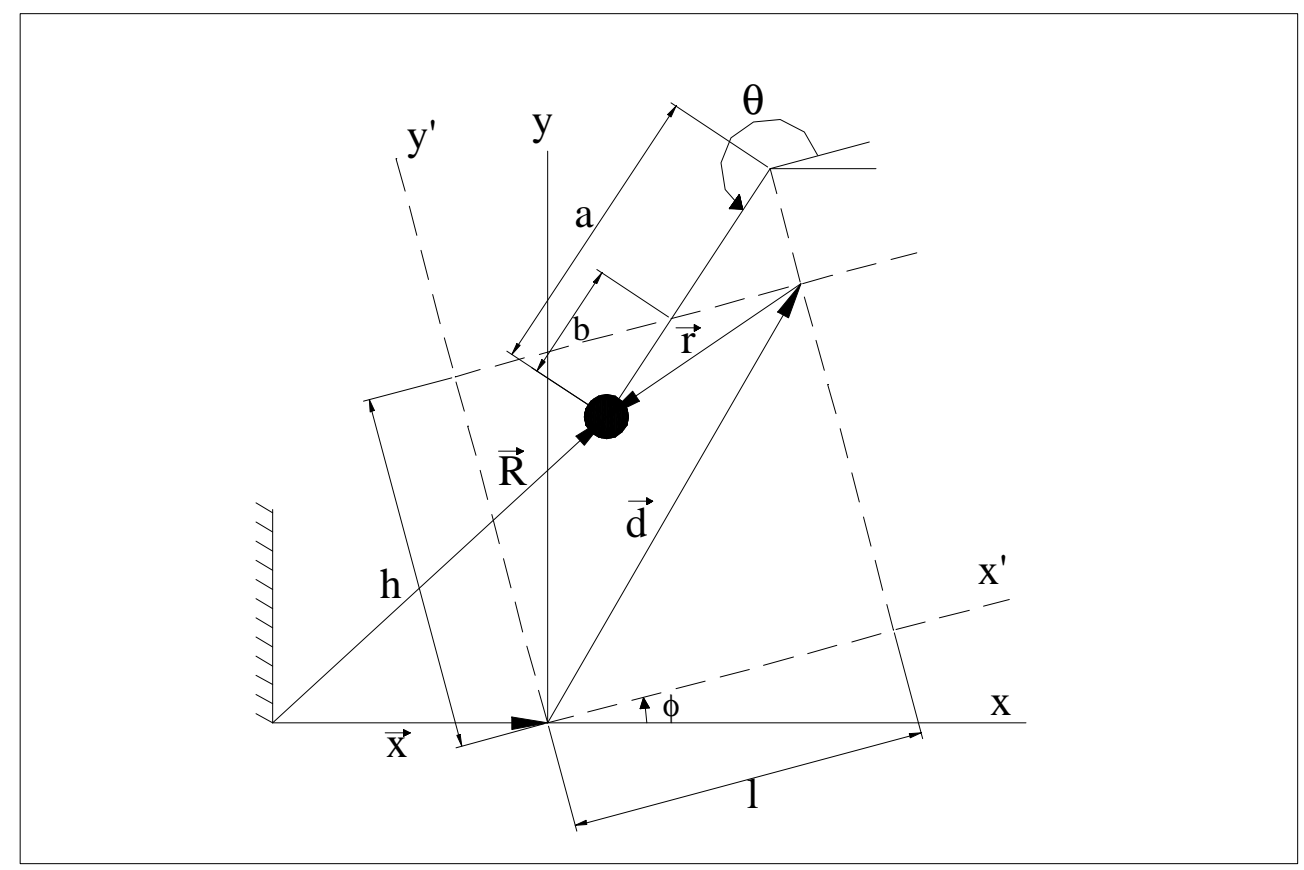

Figure 4.2: Unilateral Support System when $\phi$ is greater than Zero (ccw).

$$
\begin{aligned}
& \ddot{\theta}\left(a^{2} \sin ^{2} \theta+b^{2} \cos ^{2} \theta\right)+1 / 2 \dot{\theta}^{2}\left(a^{2}-b^{2}\right) \sin 2 \theta+\ddot{\phi}(a h \sin \theta+b l \cos \theta+a b) \\
& +\dot{\phi}^{2}\left[a l \sin \theta-b h \cos \theta+\frac{1}{2}\left(a^{2}-b^{2}\right) \sin 2 \theta\right]+g(b \cos \theta \cos \phi-a \sin \theta \sin \phi) \\
& \quad \ddot{x}(a \sin \theta \cos \phi+b \cos \theta \sin \phi)=0 \\
& \ddot{\theta}(a h \sin \theta+b l \cos \theta+a b)+\dot{\theta}^{2}(a h \cos \theta-b l \sin \theta)+\ddot{\phi}\left[(l+a \cos \theta)^{2}+(h+b \sin \theta)^{2}\right] \\
& -2 \ddot{\theta} \dot{\phi}\left[a l \sin \theta-b h \cos \theta+\frac{1}{2}\left(a^{2}-b^{2}\right) \sin 2 \theta\right]+g[(l+a \cos \theta) \cos \phi-(h+b \sin \theta) \sin \phi] \\
& -\ddot{x}[(l+a \cos \theta) \sin \phi+(h+b \sin \theta) \cos \phi]=0
\end{aligned}
$$

Equations 4-2a, and 4-2b are the two second order differential equations of motion for the system shown in Figure 4.2, and are used when $\mathrm{R}_{1 \mathrm{y}}$ (left side) is the only side touching the ground, a more detailed derivation could also be seen in the Appendix. 
By solving Equations 3-5 and 3-6 simultaneously, the values for the angle of swing $\theta$ and its angular velocity $\dot{\theta}$ were found to be equal to $5.4905 \mathrm{rad}$ and $4.6869 \mathrm{rad} / \mathrm{sec}$, respectively when the reaction at $R_{1 y}$ first became negative (system with a double degree of freedom under unilateral support). We can use these values as initial conditions along with Equations 4-1a and 4-1b to solve for stability of the system.

Figures 4.3 and 4.4 below show the values for $\theta$ and $\phi$ with respect to time under the above initial conditions of $\theta$ and $\dot{\theta}$. The system was also subjected to a lateral acceleration of $1 \mathrm{~g}$ starting at 0.075 seconds, assuming no slip condition.

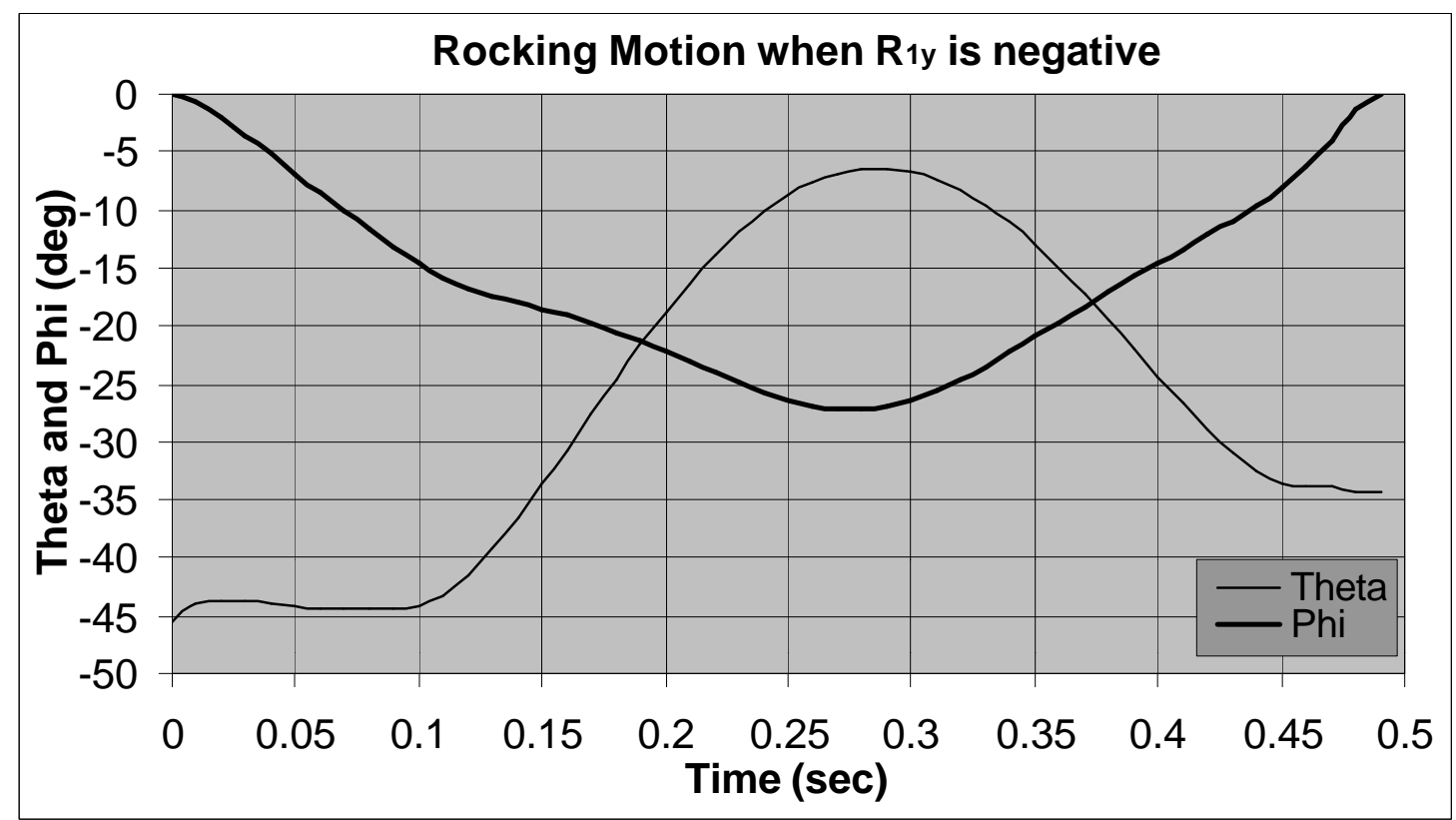

Figure 4.3: Angles of Displacement with respect to Time during Unilateral Support. 

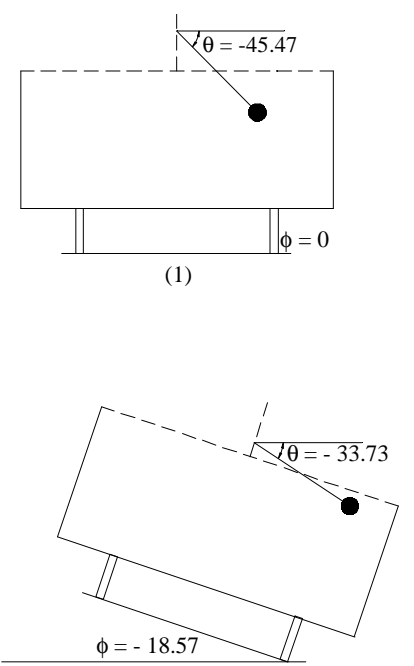

(4)
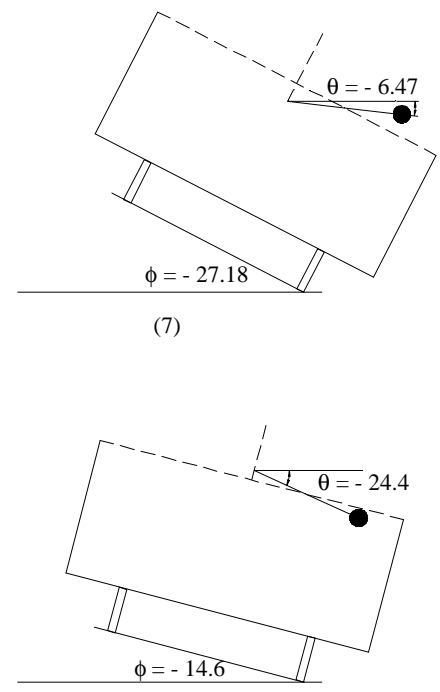

(10)
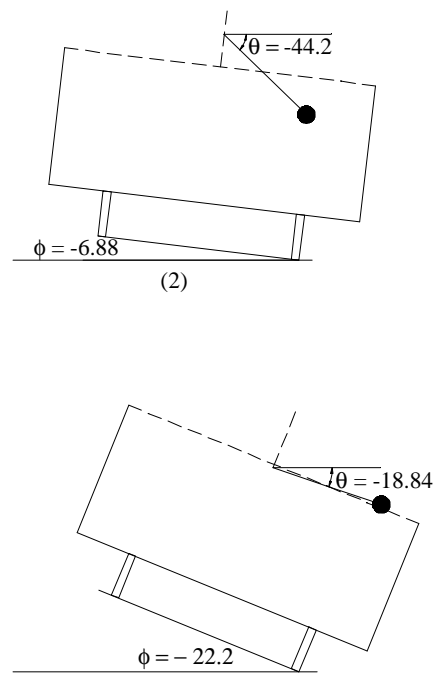

(5)
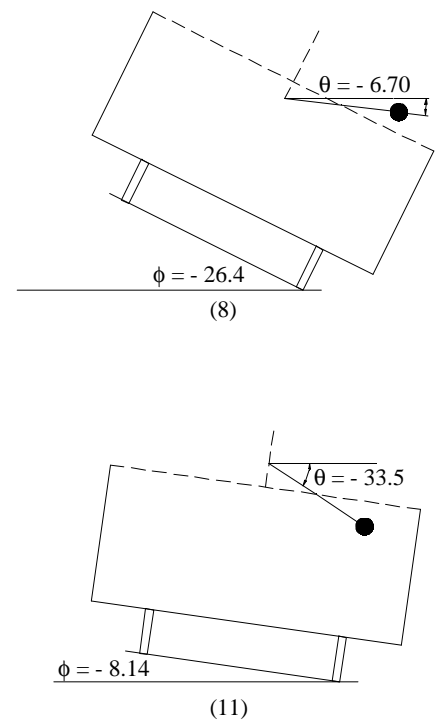
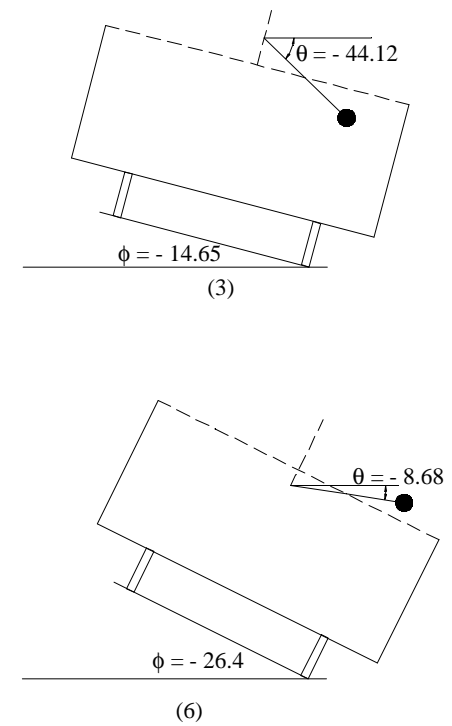

(6)
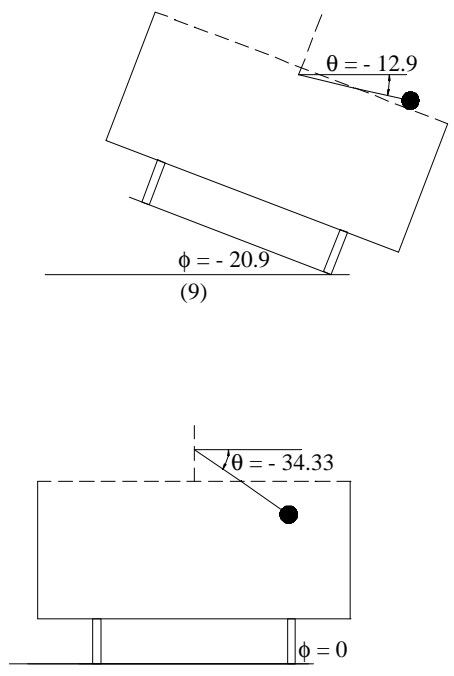

(12)

Figure 4.4: Both Angles Theta and Phi (in deg) at different Time when $\mathrm{R}_{1 \mathrm{y}}$ is Negative (measured cw).

From Figure 4.3 or 4.4 above, the minimum value for $\phi$ was found to be -27.18 degrees when $\theta$ was equal to -6.47 degrees. This concludes that the system was stable during 
the entire time. We also notice that when the value of $\phi$ returned to zero (system with one d.o.f.), the value for $\theta$ and $\dot{\theta}$ were different from the starting initial condition.

The angular velocities $\dot{\theta}$, and $\dot{\phi}$ are shown in Figures 4.5 and 4.7 respectively with respect to time. A significant change in both velocities at 0.075 seconds can me seen since the system was subjected to a lateral acceleration of positive $1 \mathrm{~g}$ at that time. The angular accelerations $\ddot{\theta}$, and $\ddot{\phi}$ are shown in Figures 4.6 and 4.8 respectively with respect to time.

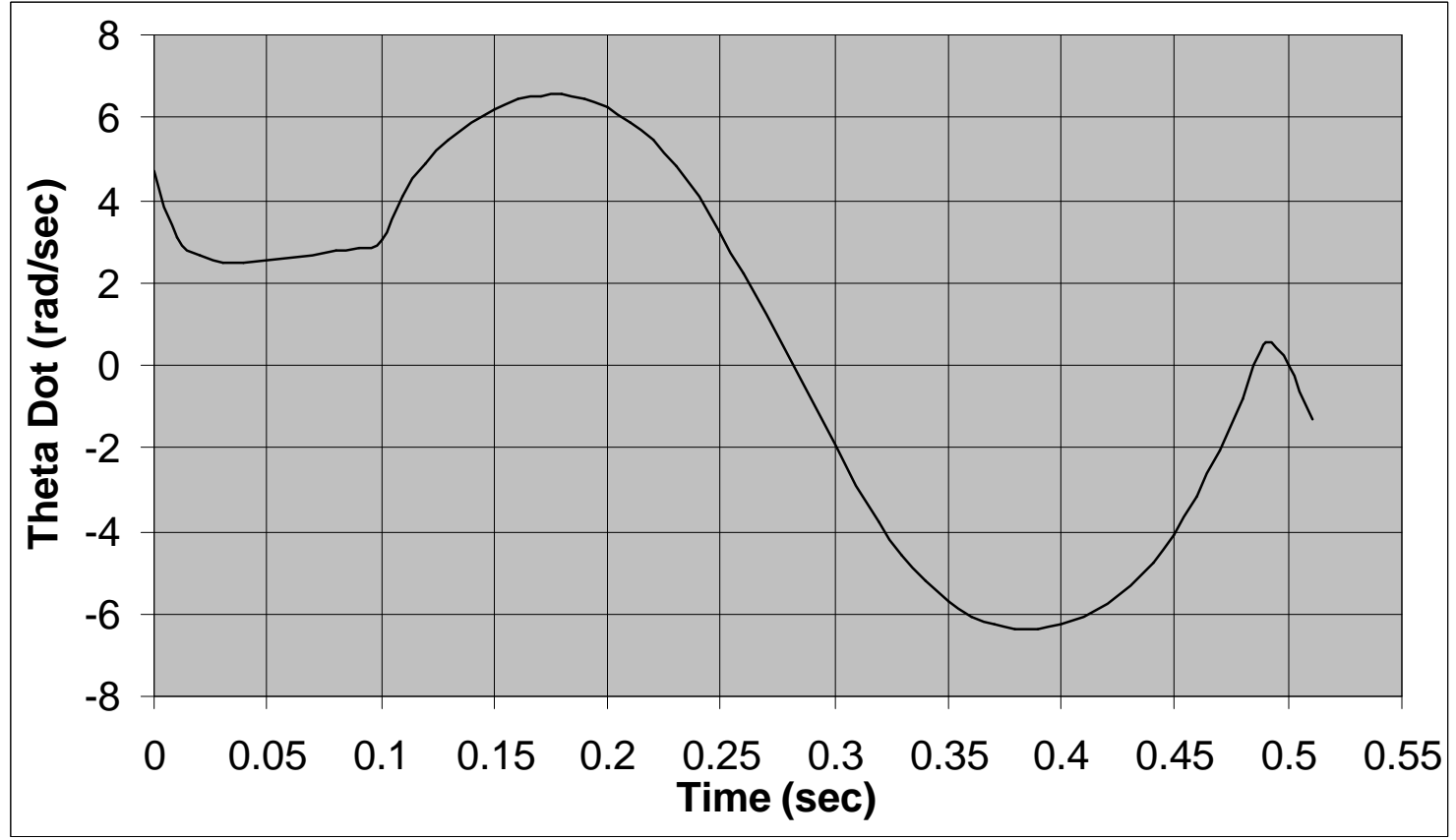

Figure 4.5: The Angular Velocity $\dot{\theta}$ with respect to Time when $\mathrm{R}_{1 \mathrm{y}}$ is Negative. 


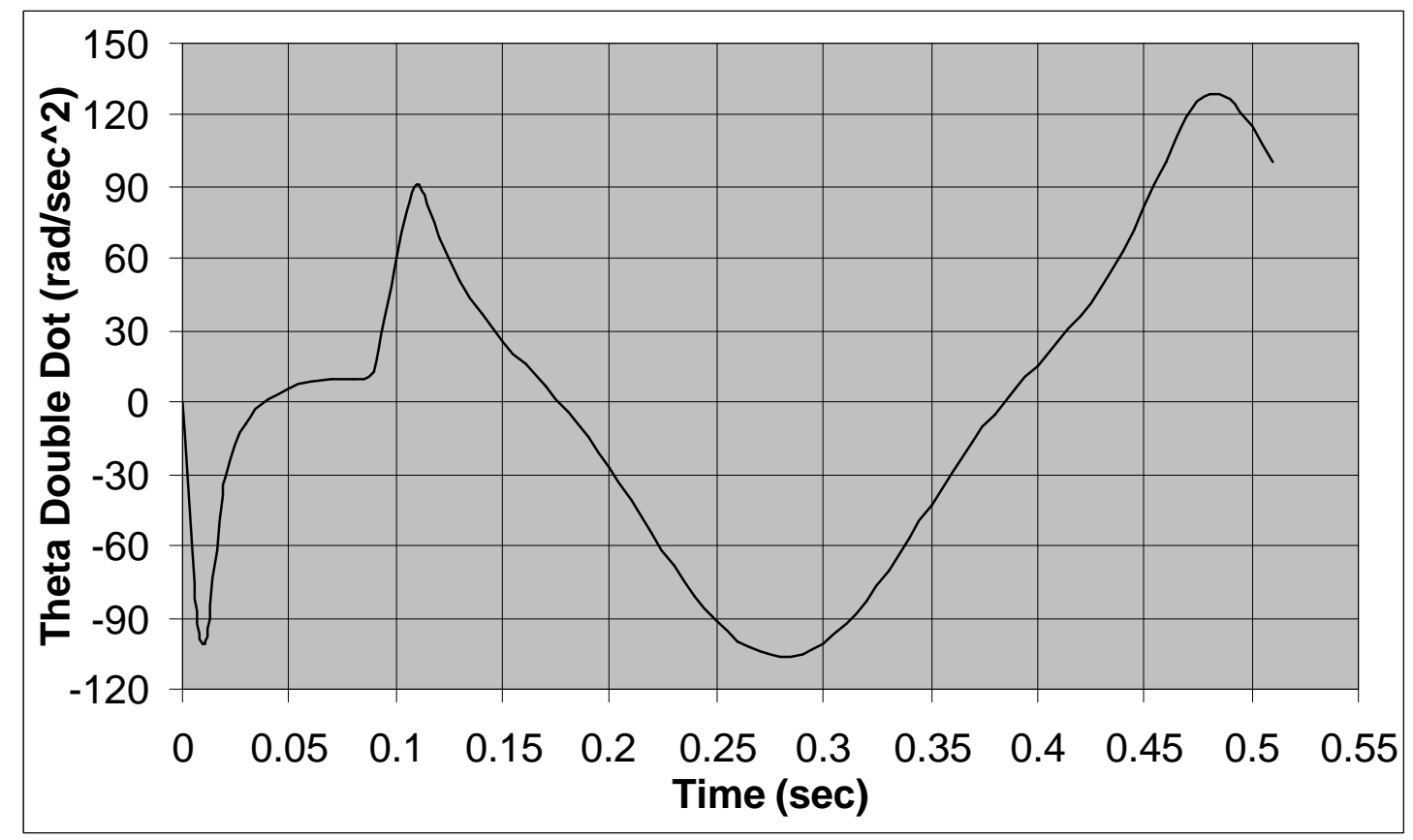

Figure 4.6: The Angular Acceleration $\ddot{\theta}$ with respect to Time when $R_{1 y}$ is Negative.

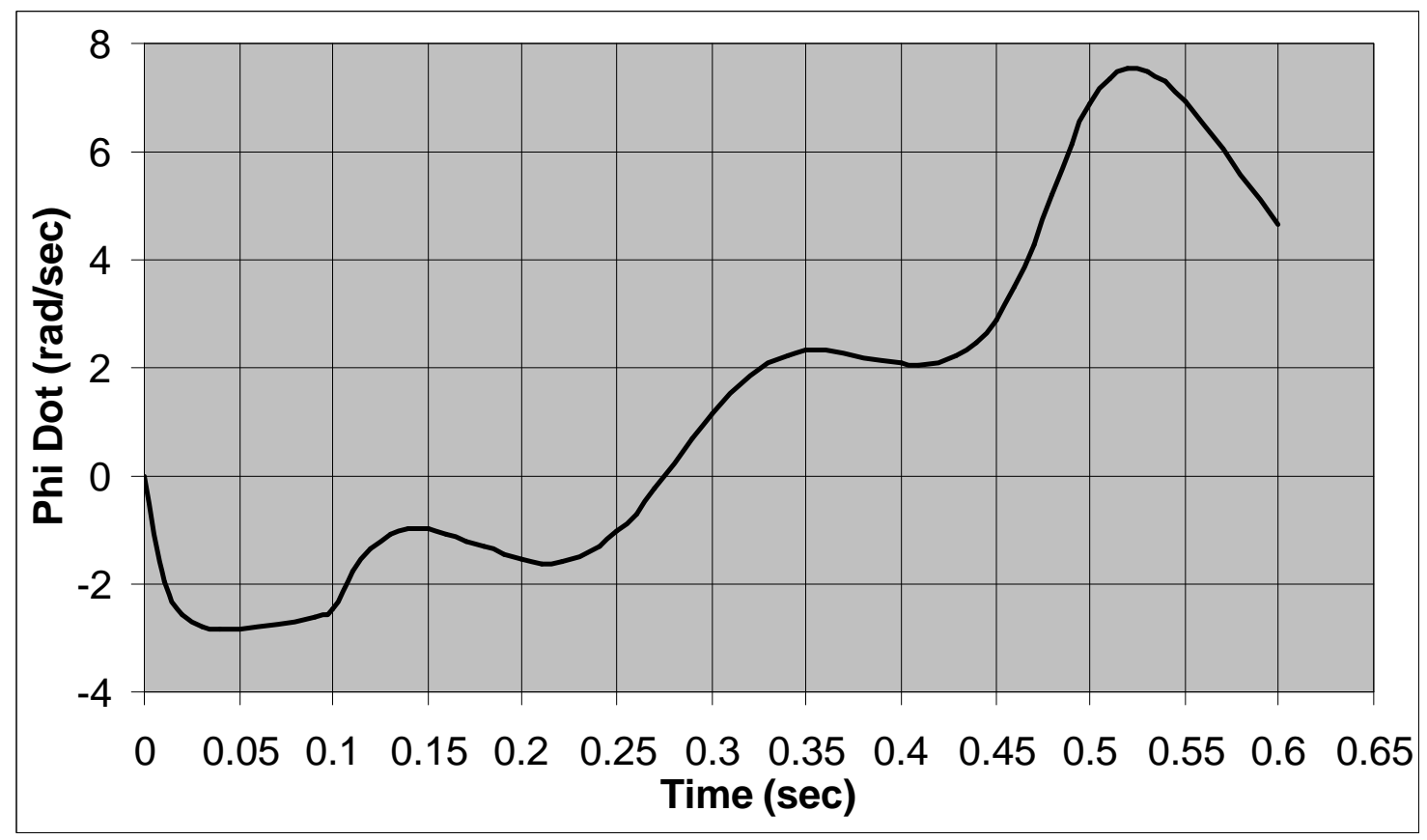

Figure 4.7: The Angular Velocity $\dot{\phi}$ with respect to Time when $R_{1 y}$ is Negative. 


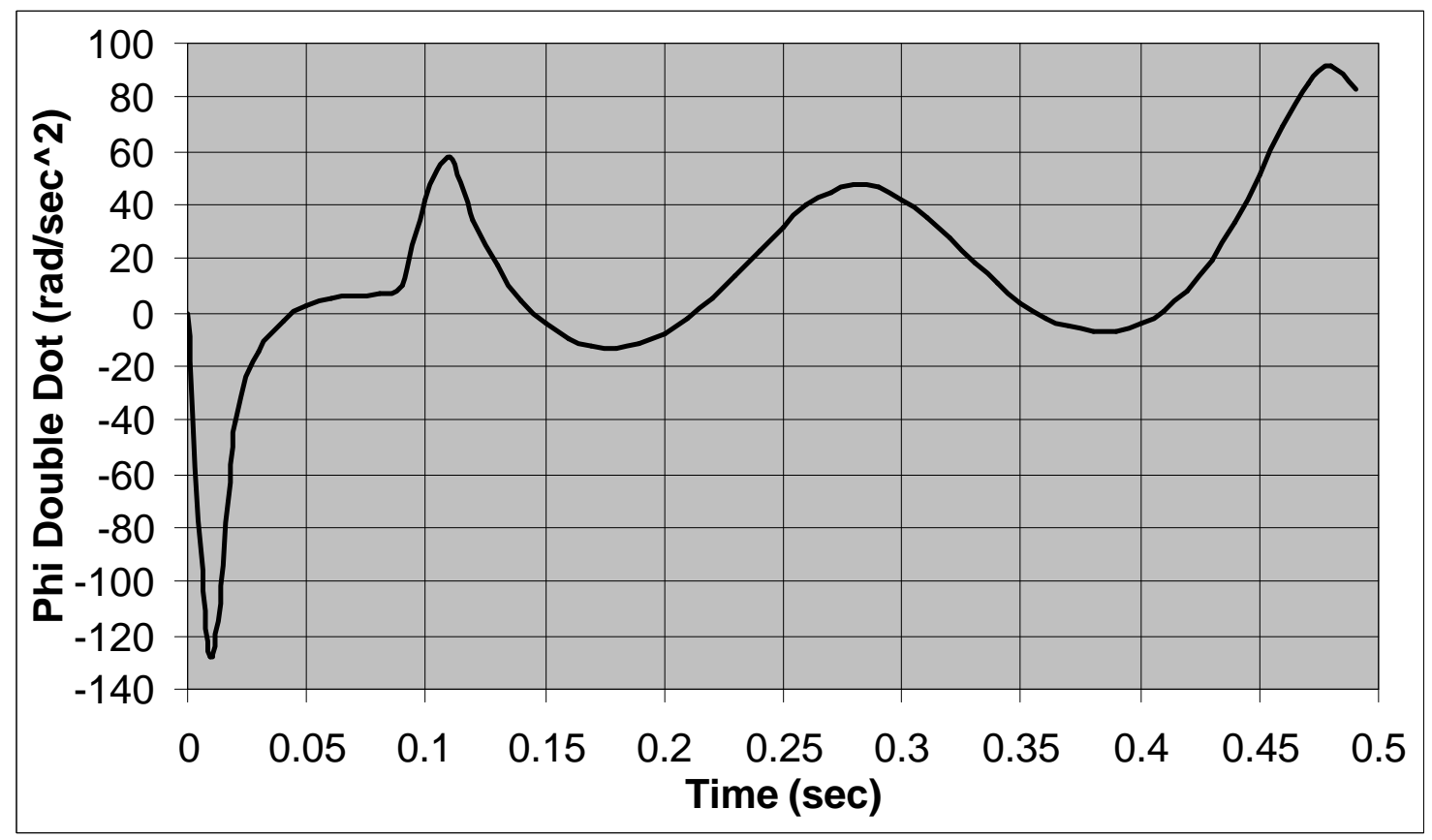

Figure 4.8: The Angular Acceleration $\ddot{\phi}$ with respect to Time when $\mathrm{R}_{1 \mathrm{y}}$ is Negative.

After the angle of lift $\phi$ returned to zero, the system returned to a single degree of freedom $\theta$. The system remained a single degree of freedom until the reaction $R_{2 y}$ became negative, at this moment the system became a double degree of freedom with $\theta$, and $\phi$ as the two unknown. Using Equations 4-2a and 4-2b with $\theta$, and $\dot{\theta}$ as initial conditions for that instant of time. The reaction at $R_{2 y}$ became negative when the angle of swing $\theta$ reached +3.94 $\operatorname{rad}(+225.5 \mathrm{deg})$, and its angular velocity $\dot{\theta} \mathrm{reached}-2.50 \mathrm{rad} / \mathrm{sec}$. 


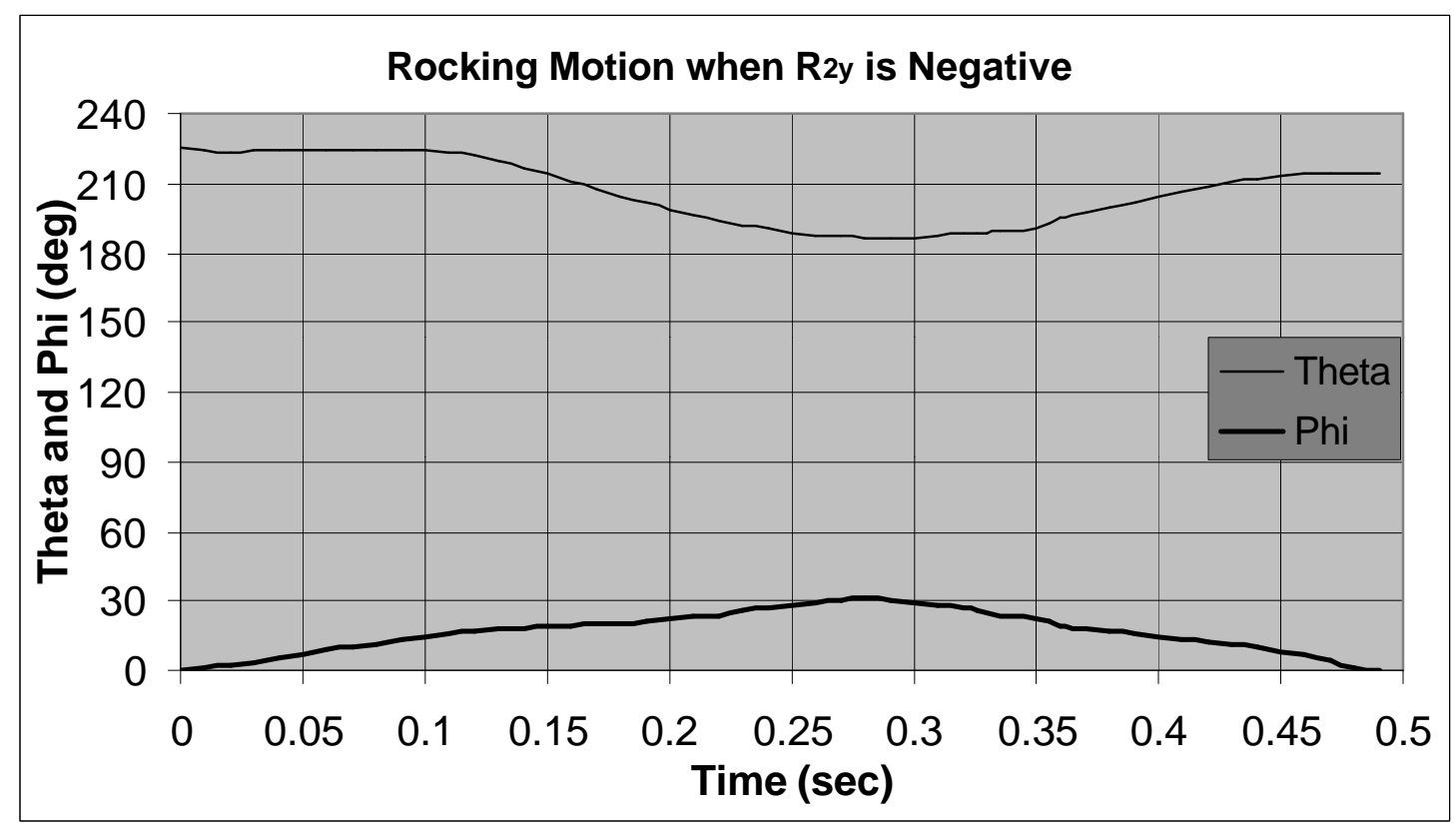

Figure 4.9: Angles of Displacement with respect to Time during Unilateral Support.

Figure 4.9 shows the angle of swing $\theta$ and the angle of lift $\phi$ both changing with respect to time. Figure 4.10 shows the numerical value of each angle at different intervals in time, it also shows the stability of the system for the entire process since the angle of lift $\phi$ increased from zero to a maximum of 30.5 degrees and then started decreasing until it returned to zero. The angular displacement $\theta$ decreased from 225.5 degrees to a minimum of 184.3 degrees and then increased to 215.6 degrees. 

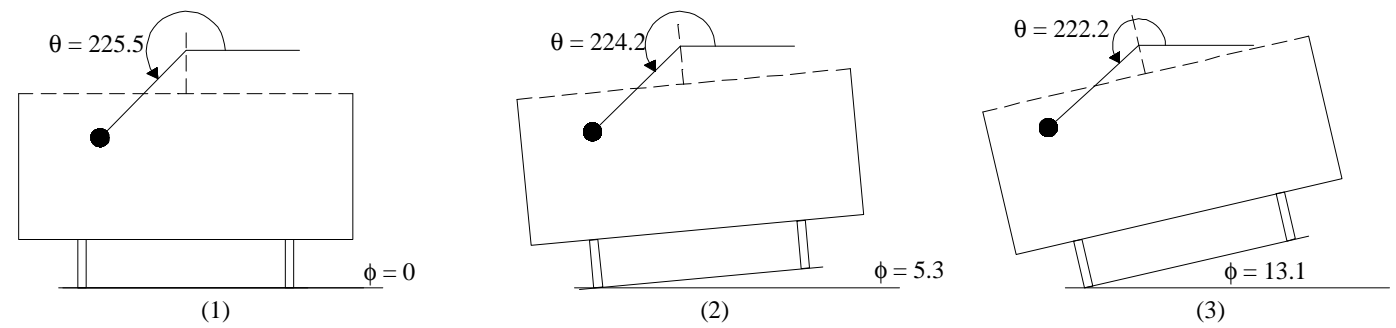

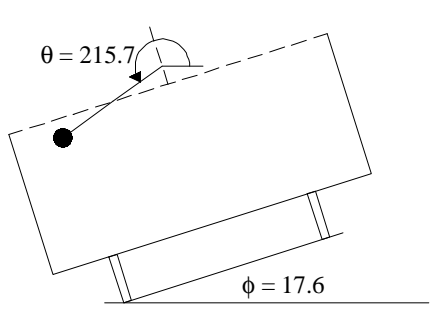

(4)
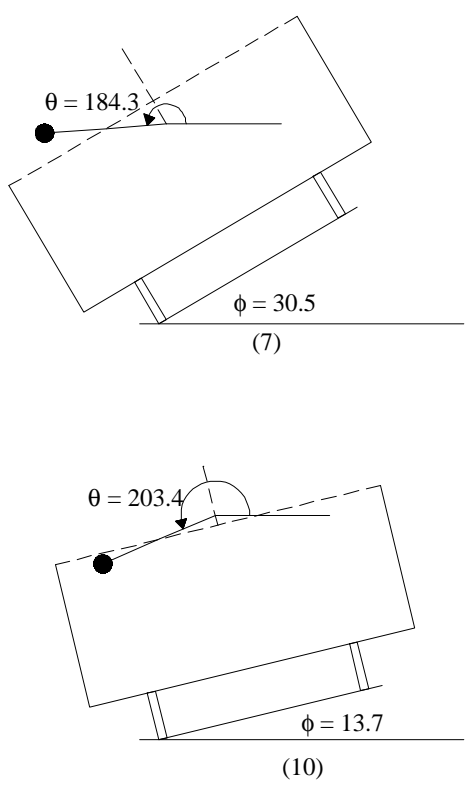

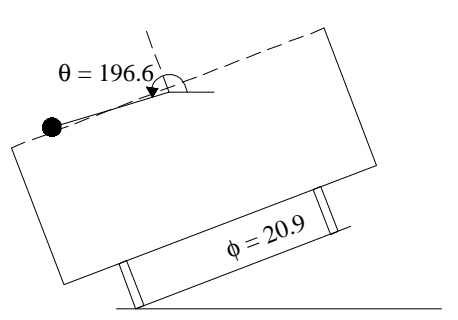

(5)
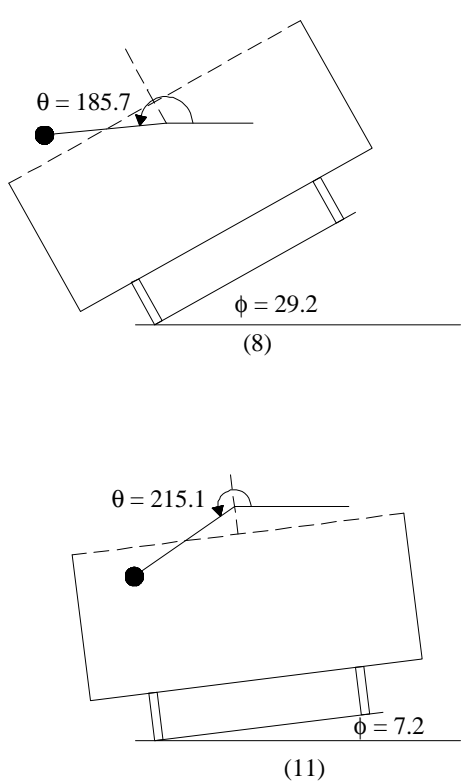

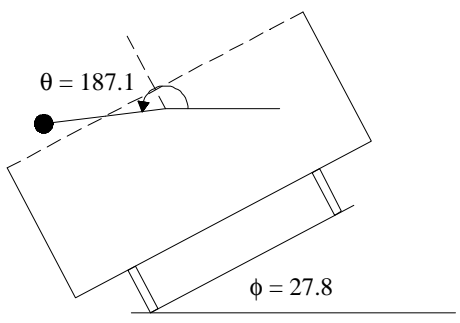

(6)
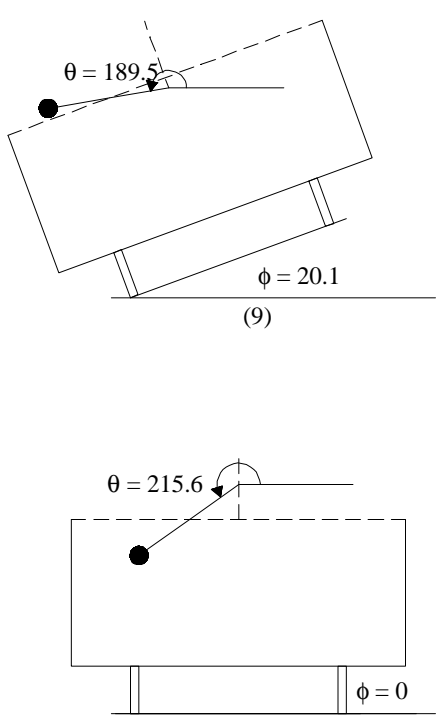

(12)

Figure 4.10: Both Angles Theta and Phi (in deg) at different Times when $R_{2 y}$ is Negative (measured ccw). 


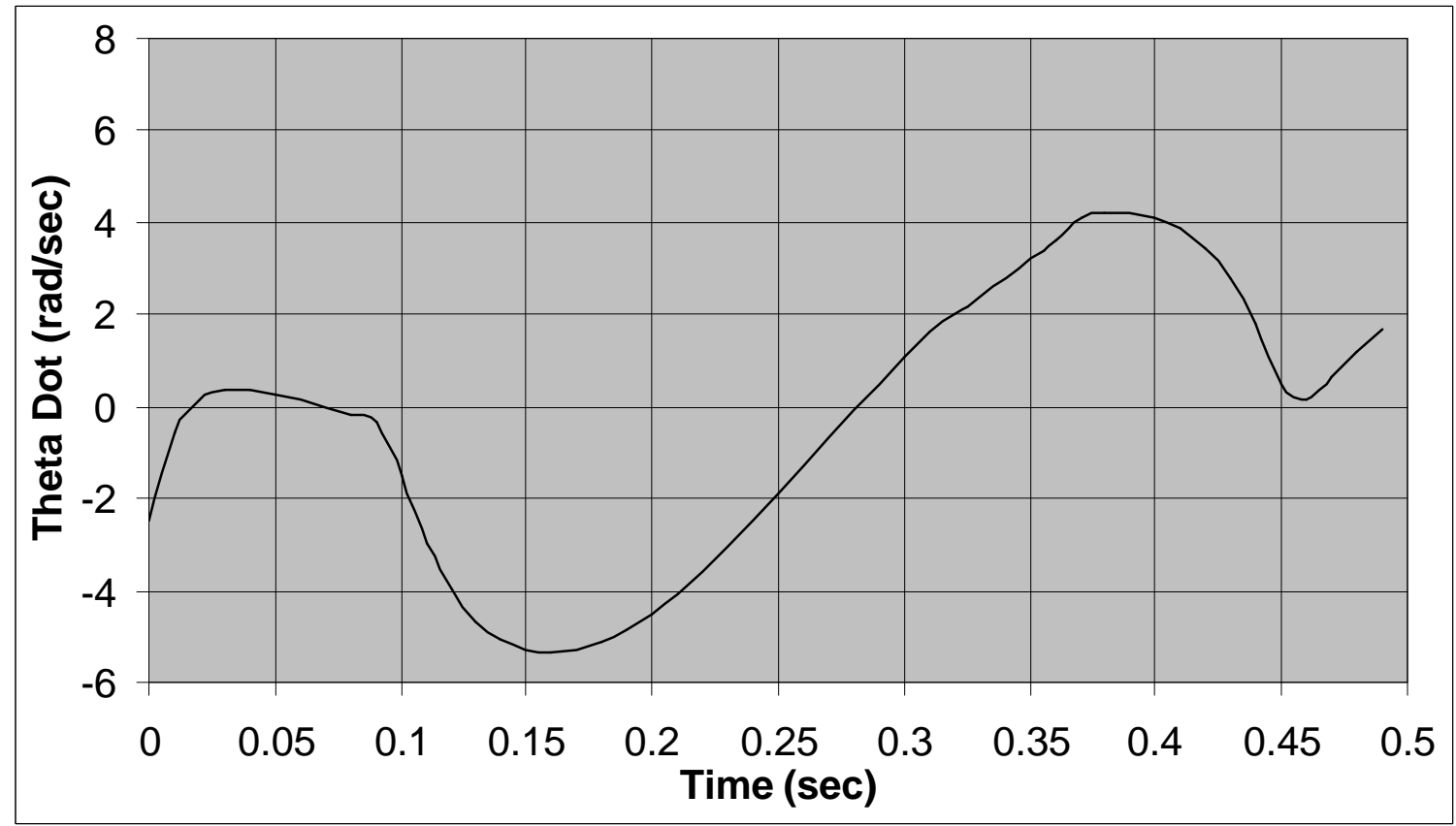

Figure 4.11: The Angular Velocity $\theta$ with respect to Time when $R_{2 y}$ is Negative.

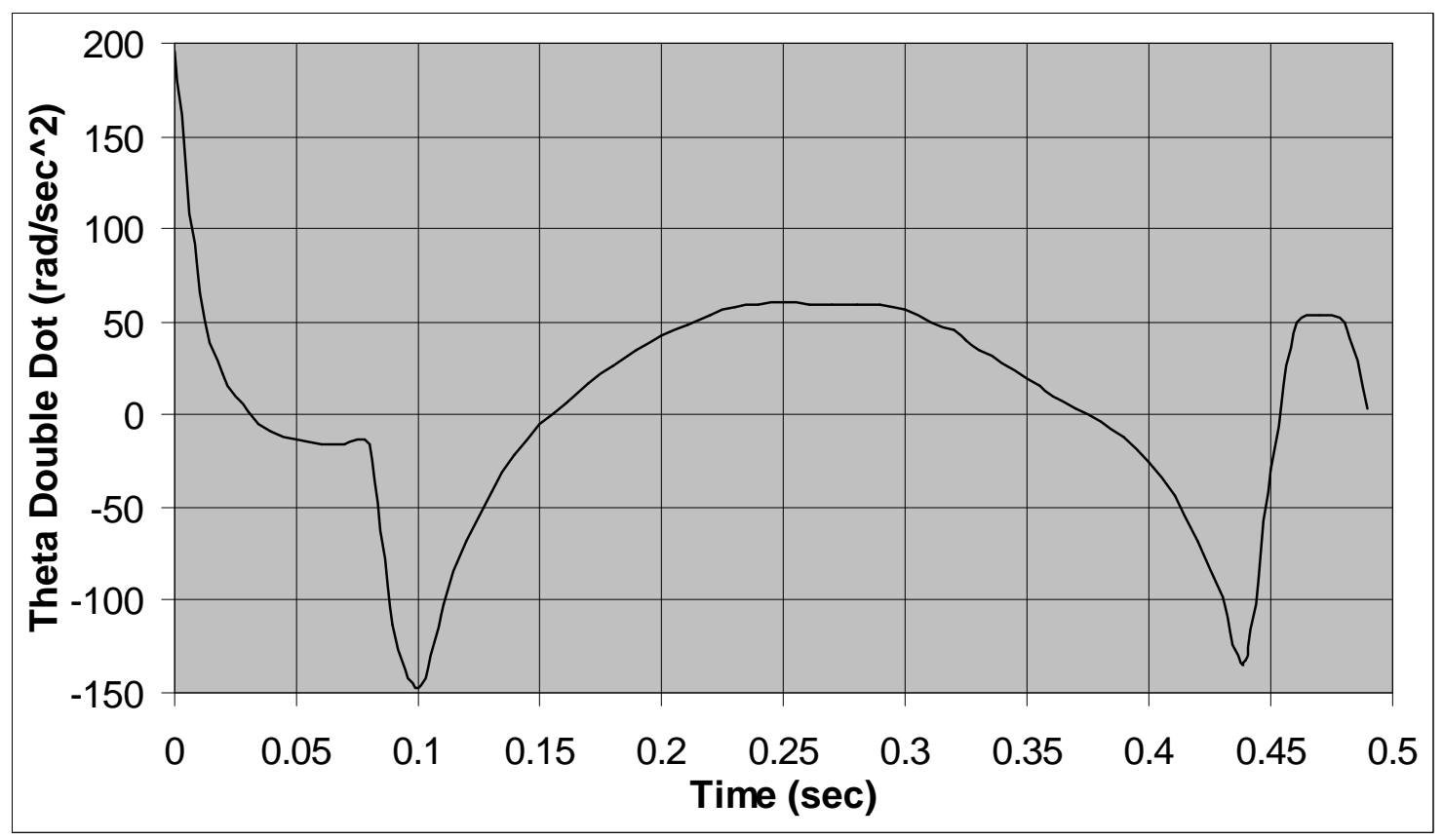

Figure 4.12: The Angular Acceleration $\ddot{\theta}$ with respect to Time when $R_{2 y}$ is Negative. 


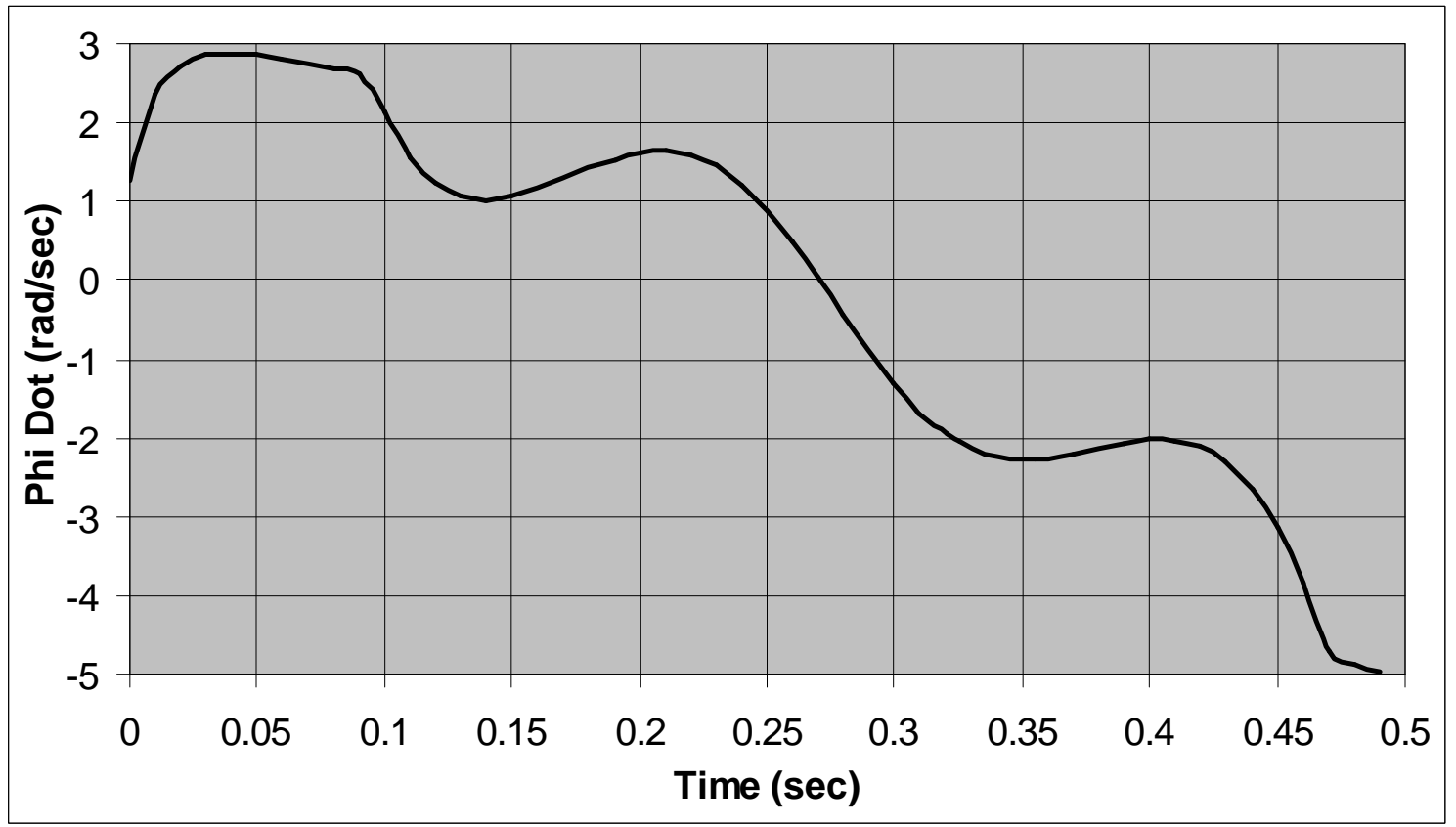

Figure 4.13: The Angular Velocity $\phi$ with respect to Time when $R_{2 y}$ is Negative.

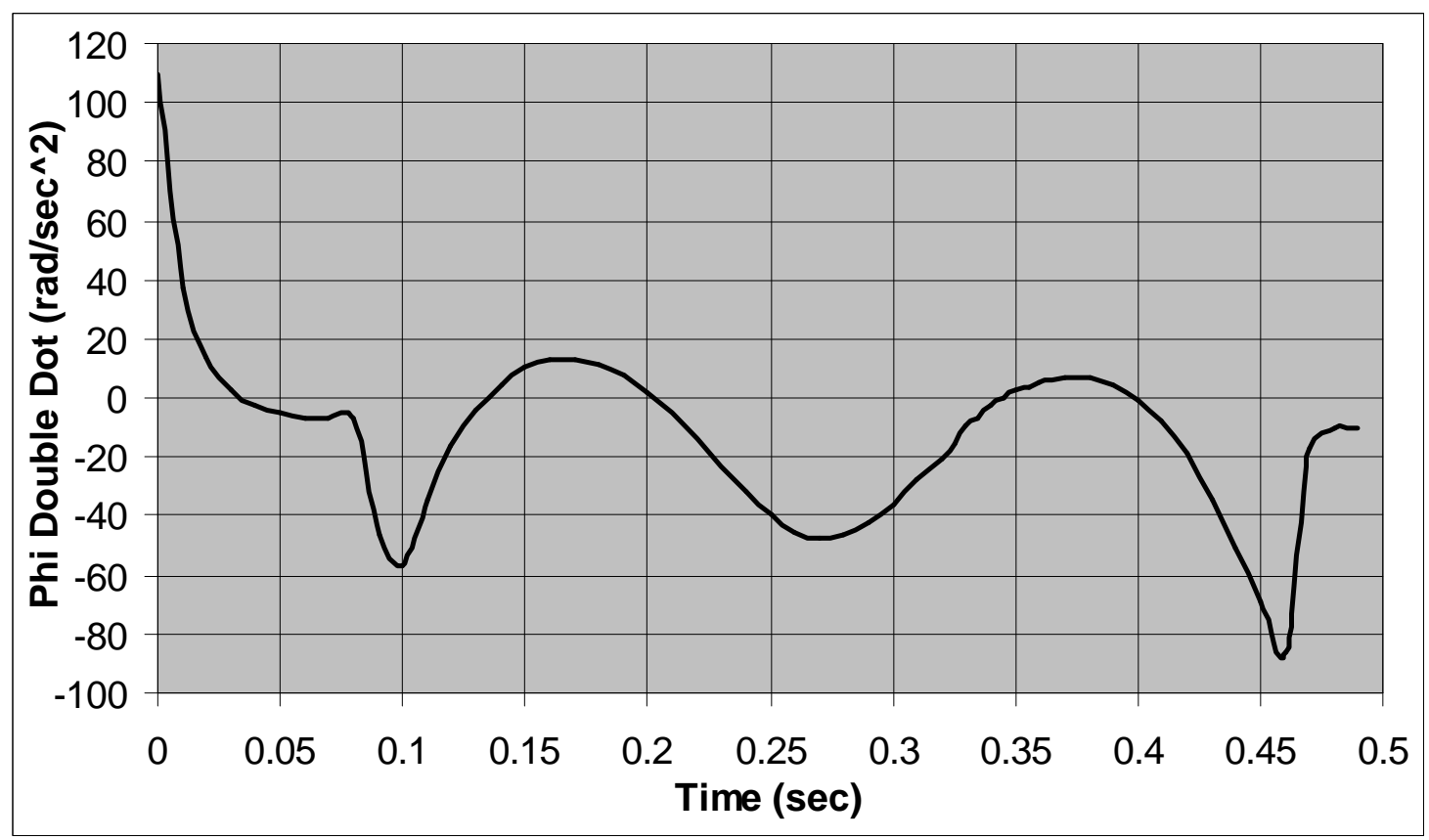

Figure 4.14: The Angular Acceleration $\ddot{\phi}$ with respect to Time when $R_{2 y}$ is Negative. 
Figures 4.11 and 4.13 show the angular velocities curves for both angles $\theta$ and $\phi$ respectively with respect to time, while Figures 4.12 and 4.14 show their angular accelerations with respect to time.

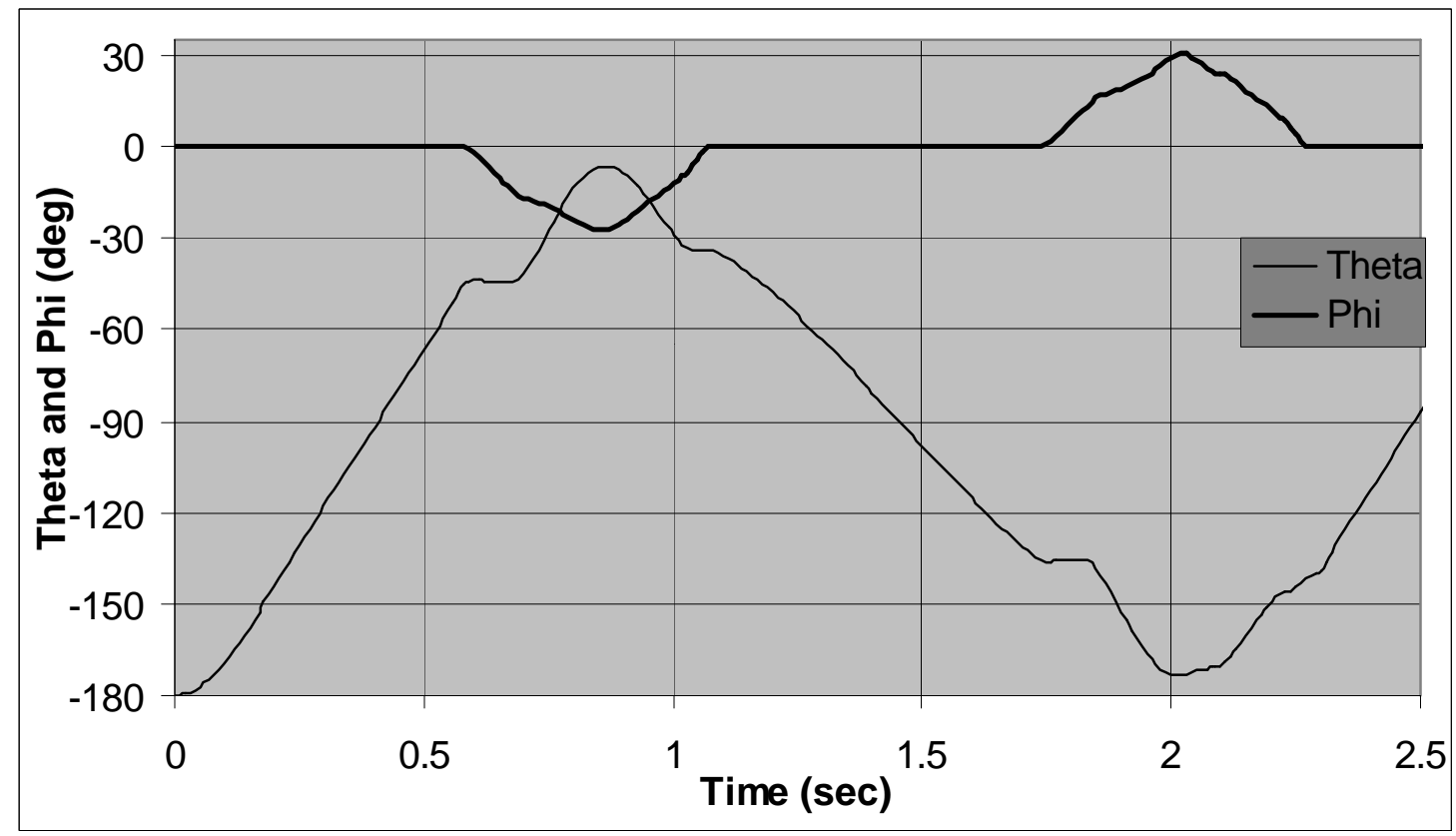

Figure 4.15: Both Angular Displacements with respect to Time during One Complete Cycle.

Figure 4.15 shows both angular displacements $\theta$ and $\phi$ during a complete cycle starting with $\theta$ equal to 180 degrees and $\phi$ equal to zero (system has both supports touching the ground). Both angles were measured from the horizontal axis (positive when measured counterclockwise and negative when measured clockwise). The system lost contact with the ground at $\mathrm{R}_{1 \mathrm{y}}$ when $\theta$ became greater than 314.5 degrees, and became unilateral ( 2 d.o.f.). The angle of tilt $\phi$ started to increase in magnitude until it reached its maximum of 27.18 degrees (cw), $\theta$ also reached its maximum of 353.5 degrees (ccw) and then it started to decrease again, decreasing $\phi$ in magnitude at the same time until it reached zero. Hence, the 
system returned to 1 d.o.f. having $\theta$ as the only unknown. It remained 1 d.o.f. system until $\theta$ reached 225.5 degrees. At 225.5 degrees, the reaction in the right side $\left(\mathrm{R}_{2 \mathrm{y}}\right)$ became negative causing it to lift off the ground, which caused $\phi$ to increase in magnitude until it reached its maximum of 30.53 degrees (ccw) before returning to zero. When the angle of tilt $\phi$ reached its maximum, the angle of swing $\theta$ reached its minimum of 184.3 degrees (ccw), then started to increase in magnitude.

\subsection{System during Unstable Condition}

If the system was subject to a lateral acceleration of negative $1 \mathrm{~g}$ while it was supported by the right side $\left(\mathrm{R}_{2 \mathrm{y}}\right.$ is the only reaction touching the ground), the system will become unstable. Hence, rollover of the tank.

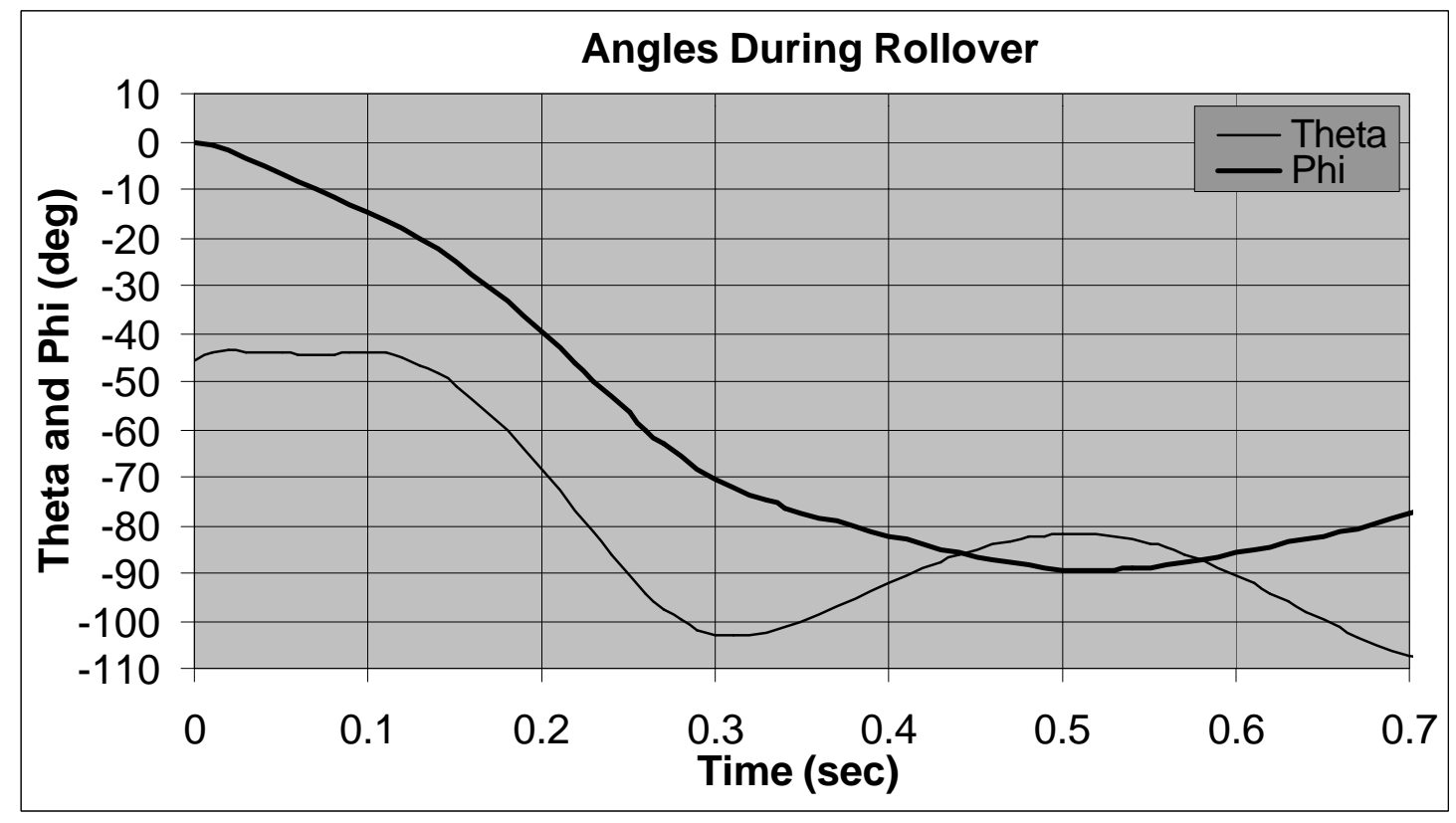

Figure 4.16: Both Angles with respect to Time during Rollover. 


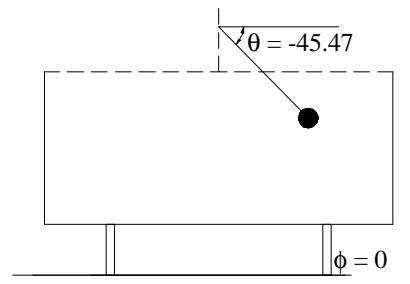

(1)
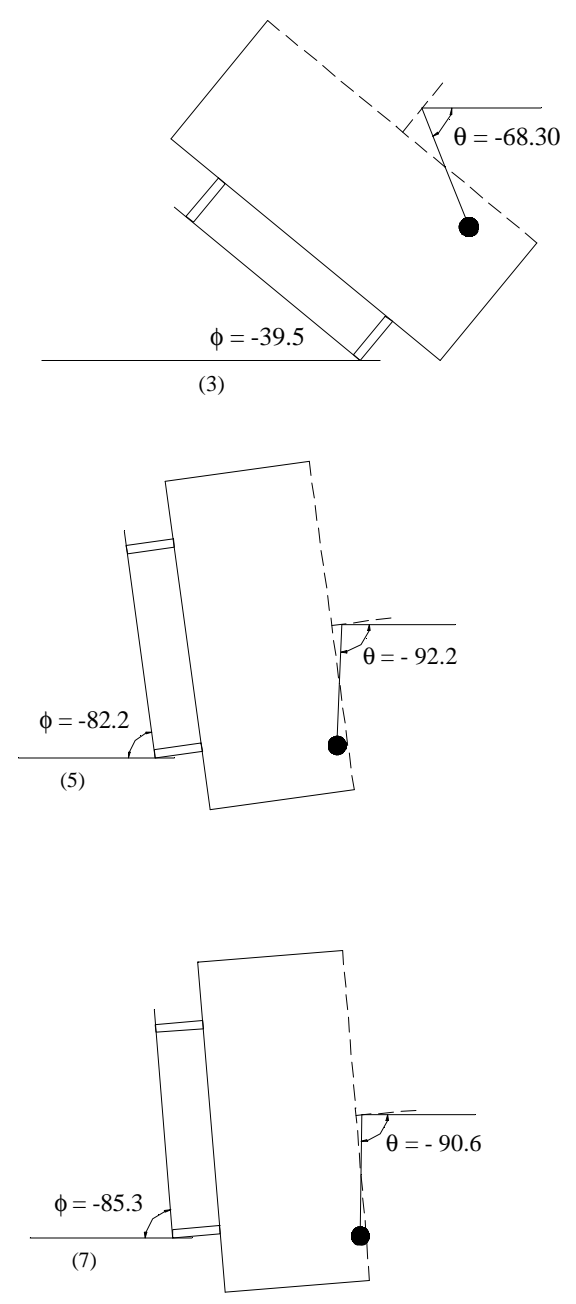
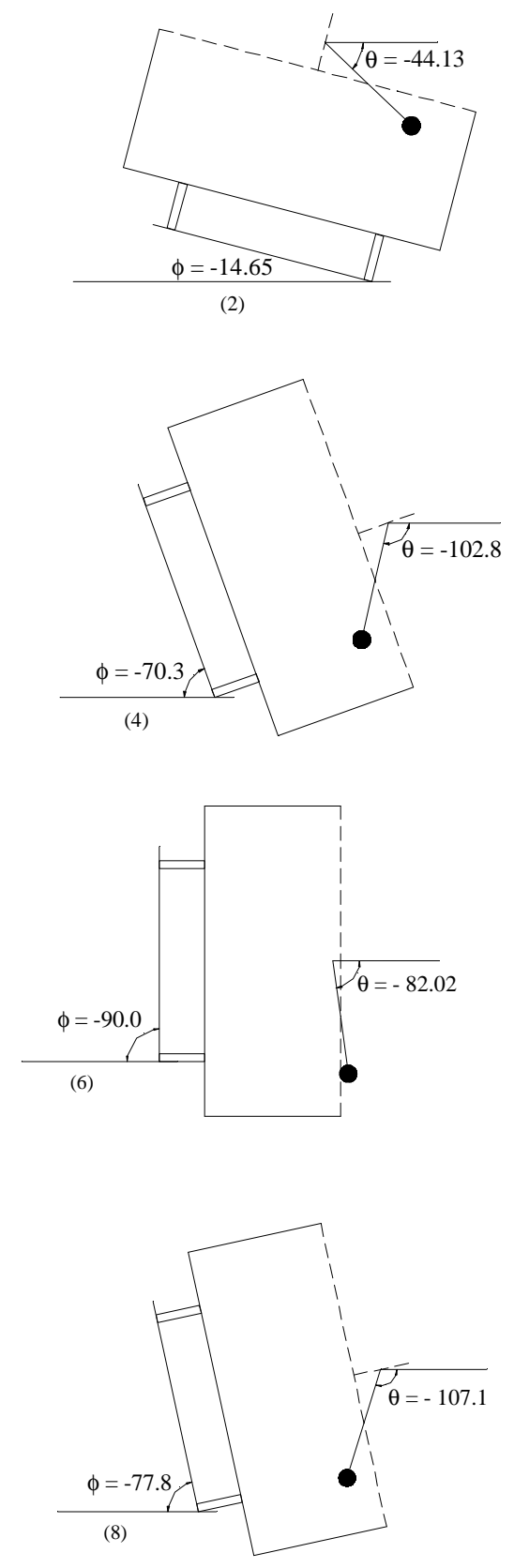

Figure 4.17: Both Angles (in deg) when Rollover Occurs (measured cw).

Figures 4.16 and 4.17 above show the values for $\theta$ and $\phi$ with respect to time during an unstable system. We noticed that the angle of swing $\theta$ decreased to its minimum of -102.8 
degrees when $\phi$ was at -70.3 degrees (case 4 in Figure 4.17), but started increasing again until $\phi$ reached its maximum of -90.0 degrees. Both angles started to decrease again, causing the tank to roll back towards its original supports. 


\section{CHAPTER V}

\section{DISCUSSION OF RESULTS AND CONCLUSIONS}

\subsection{Energy Equations}

One way of checking the solutions is by studying the total energy of the system. The system should have constant energy throughout the entire process. Equations 5-1 and 5-2 show the kinetic and potential energy of the system respectively.

$$
\begin{aligned}
& T=\frac{1}{2} m\left(a^{2} \sin ^{2} \theta+b^{2} \cos ^{2} \theta\right) \\
& V=m g(h+b \sin \theta)
\end{aligned}
$$

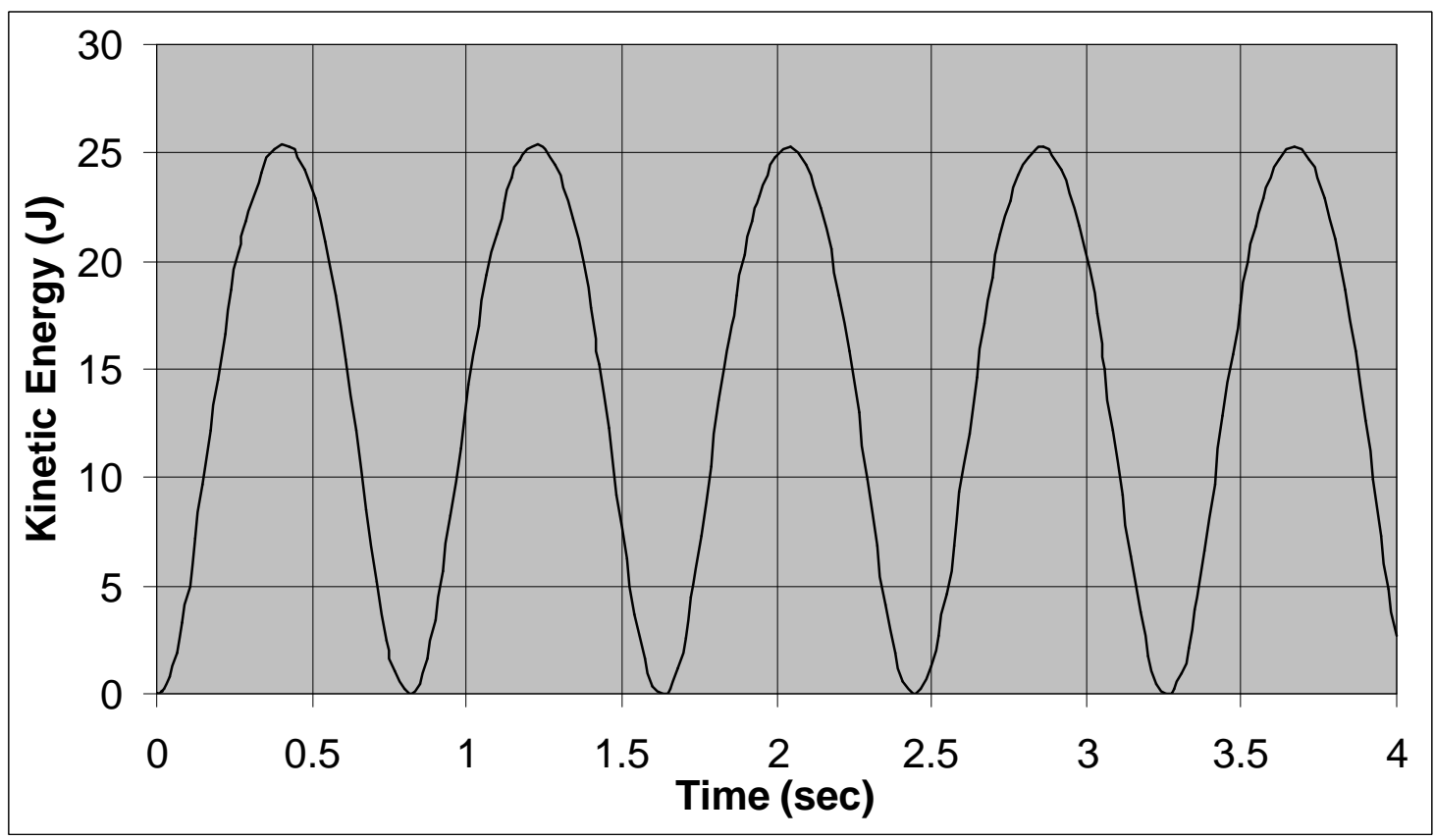

Figure 5.1: Kinetic Energy of the System with respect to Time. 


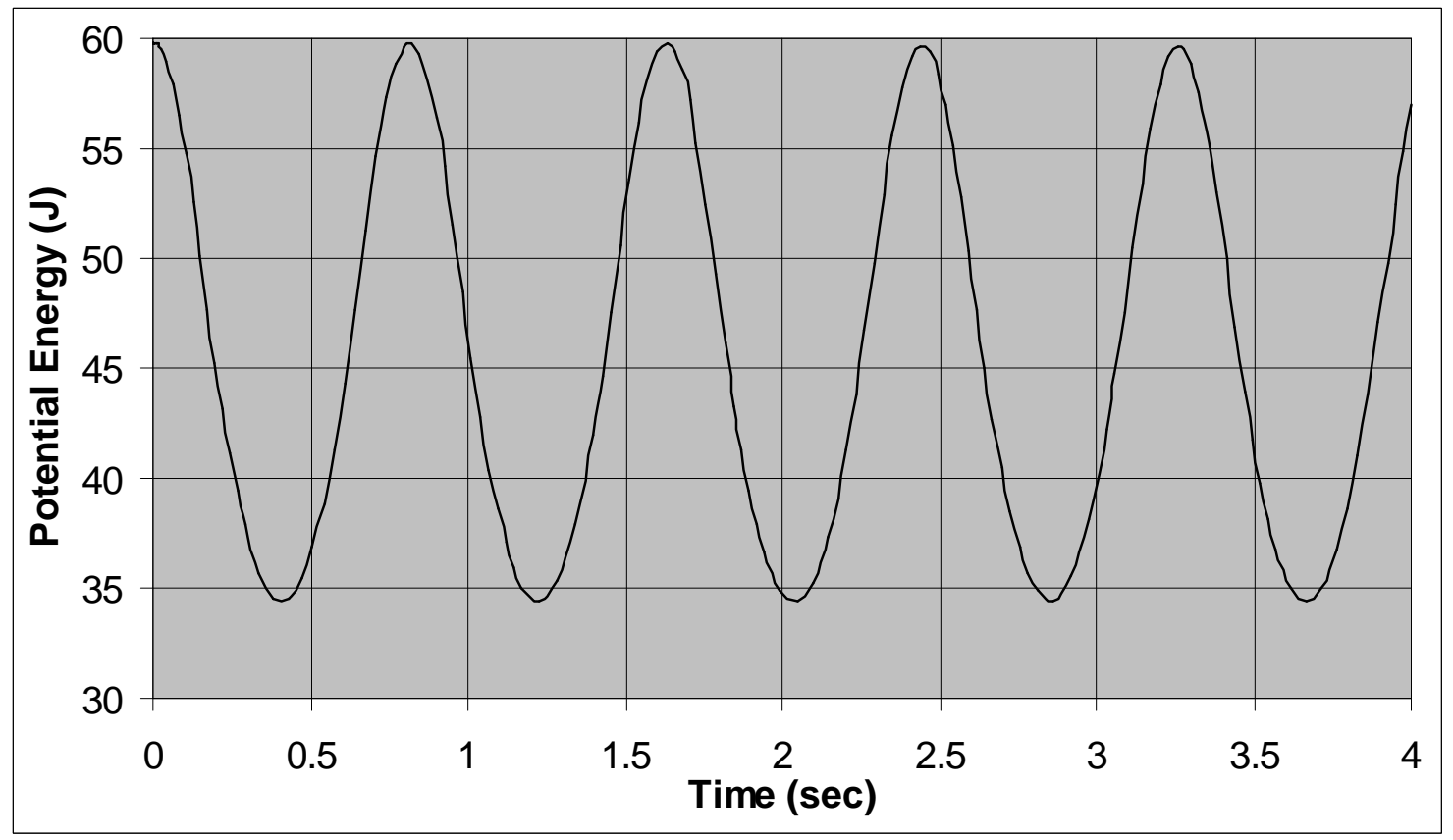

Figure 5.2: Potential Energy of the System with respect to Time.

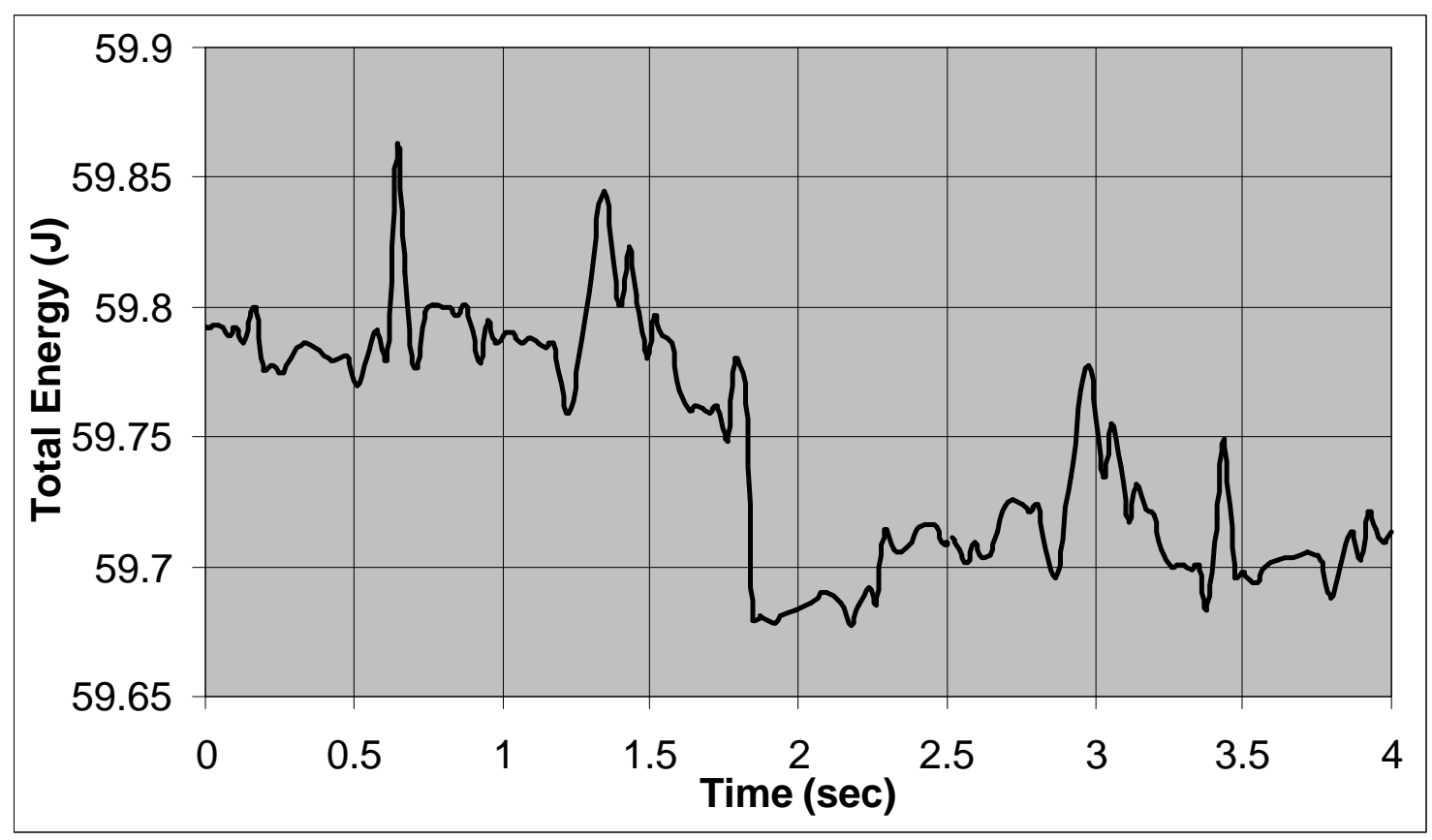

Figure 5.3: Total Energy of the System with respect to Time. 
As we can see from the figures above, the potential energy varied between a maximum of $59.8 \mathrm{~J}$ and a minimum of $34.5 \mathrm{~J}$. The kinetic energy varied between a minimum of $0.0 \mathrm{~J}$ and a maximum of $25.3 \mathrm{~J}$ (decreasing when the kinetic energy is increasing and vice versa). From Figure 5.3, the percent error of the total energy was calculated and found to be equal to $0.3 \%$ which could be attributed to the absolute and relative tolerance of the numerical method. Since the percent error was small, it was assumed to be negligible. The mean value of the total energy was also calculated and found to be equal to $59.75 \mathrm{~J}$. This indicated that the analysis carried out produced feasible results.

\subsection{Discussion of Results}

The elliptical tank dynamic simulation tests showed reasonable results according to the initial conditions applied to the elliptical pendulum. Since the equations of motion were of higher order nonlinear differential equations, the graphs for the angular displacement, velocity and acceleration with respect to time show higher order variations which are visible at the peaks and inflection points.

Table 5.1 shows the critical angle in which the pendulum swings producing liftoff but not rollover for a system with zero lateral acceleration, and the minimum horizontal lateral acceleration that produces liftoff for a system initially at rest. An example of such lateral acceleration is when the tanker vehicle is going around a curve with a small radius of curvature at high speed. The major effect on the stability of the system was the distance between the two supports, and the length of the pendulum arm. Figures 5.6 and 5.7 show the minimum lateral acceleration and angle of swing, respectively, for different values of $\mathrm{a} / \mathrm{b}$ and $\mathrm{l} / \mathrm{h}$ that will cause 
the base to liftoff (system is initially at rest). Figure 5.8 shows the critical angle of swing of the pendulum while subjected to zero lateral acceleration.

Table 5.1:Critical Angles of Swing and Lateral Acceleration that will cause the System's Base to Liftoff (Under Different Initial Geometric Parameters)

\begin{tabular}{|c|c|c|c|c|c|c|c|c|}
\hline $\mathbf{a}$ & $\mathbf{b}$ & $\mathbf{h}$ & $\mathbf{l}$ & $\mathbf{a} / \mathbf{b}$ & $\mathbf{h} / \mathbf{l}$ & $\mathbf{X 2 d o t}$ & $\mathbf{T h e t a}$ & Theta (Deg) \\
\hline $\mathbf{c m}$ & $\mathbf{c m}$ & $\mathbf{c m}$ & $\mathbf{c m}$ & & & $\mathbf{N} / \mathbf{s}^{\wedge} \mathbf{2}$ & $\mathbf{D e g}$ & (Zero Lat. Acc.) \\
\hline & & & & & & & & \\
\hline 0.5093 & 0.2587 & 0.9 & 0.6095 & 1.96869 & 1.47662 & 0.378 & 73.3 & 76.5 \\
\hline 0.5093 & 0.3 & 0.9 & 0.6095 & 1.69767 & 1.47662 & 0.372 & 72.4 & 75 \\
\hline 0.5093 & 0.35 & 0.9 & 0.6095 & 1.45514 & 1.47662 & 0.365 & 71.4 & 74 \\
\hline 0.5093 & 0.4 & 0.9 & 0.6095 & 1.27325 & 1.47662 & 0.363 & 71.1 & 72.9 \\
\hline 0.5093 & 0.45 & 0.9 & 0.6095 & 1.13178 & 1.47662 & 0.359 & 70.5 & 72.2 \\
\hline 0.5093 & 0.5093 & 0.9 & 0.6095 & 1 & 1.47662 & 0.356 & 69.7 & 71.2 \\
\hline 0.55 & 0.2587 & 0.9 & 0.6095 & 2.12601 & 1.47662 & 0.354 & 69.7 & 73.6 \\
\hline & & & & & & & & \\
\hline 0.5093 & 0.2587 & 0.9 & 0.8 & 1.96869 & 1.125 & 0.363 & 71.1 & 75 \\
\hline 0.5093 & 0.3 & 0.9 & 0.8 & 1.69767 & 1.125 & 0.359 & 70.5 & 71.9 \\
\hline 0.5093 & 0.35 & 0.9 & 0.8 & 1.45514 & 1.125 & 0.355 & 69.9 & 71.1 \\
\hline 0.5093 & 0.4 & 0.9 & 0.8 & 1.27325 & 1.125 & 0.351 & 69.8 & 71 \\
\hline 0.5093 & 0.5093 & 0.9 & 0.8 & 1 & 1.125 & 0.342 & 67.9 & 68.4 \\
\hline 0.55 & 0.2587 & 0.9 & 0.8 & 2.12601 & 1.125 & 0.342 & 67.9 & 69.8 \\
\hline & & & & & & & & \\
\hline 0.5093 & 0.2587 & 0.8 & 0.6095 & 1.96869 & 1.31255 & 0.333 & 66.5 & 70.3 \\
\hline 0.5093 & 0.3 & 0.8 & 0.6095 & 1.69767 & 1.31255 & 0.33 & 66 & 69 \\
\hline 0.5093 & 0.35 & 0.8 & 0.6095 & 1.45514 & 1.31255 & 0.325 & 65.2 & 67.3 \\
\hline 0.5093 & 0.4 & 0.8 & 0.6095 & 1.27325 & 1.31255 & 0.32 & 64.4 & 67.1 \\
\hline 0.5093 & 0.5093 & 0.8 & 0.6095 & 1 & 1.31255 & 0.31 & 62.8 & 64.2 \\
\hline 0.55 & 0.2587 & 0.8 & 0.6095 & 2.12601 & 1.31255 & 0.308 & 62.4 & 66.9 \\
\hline
\end{tabular}




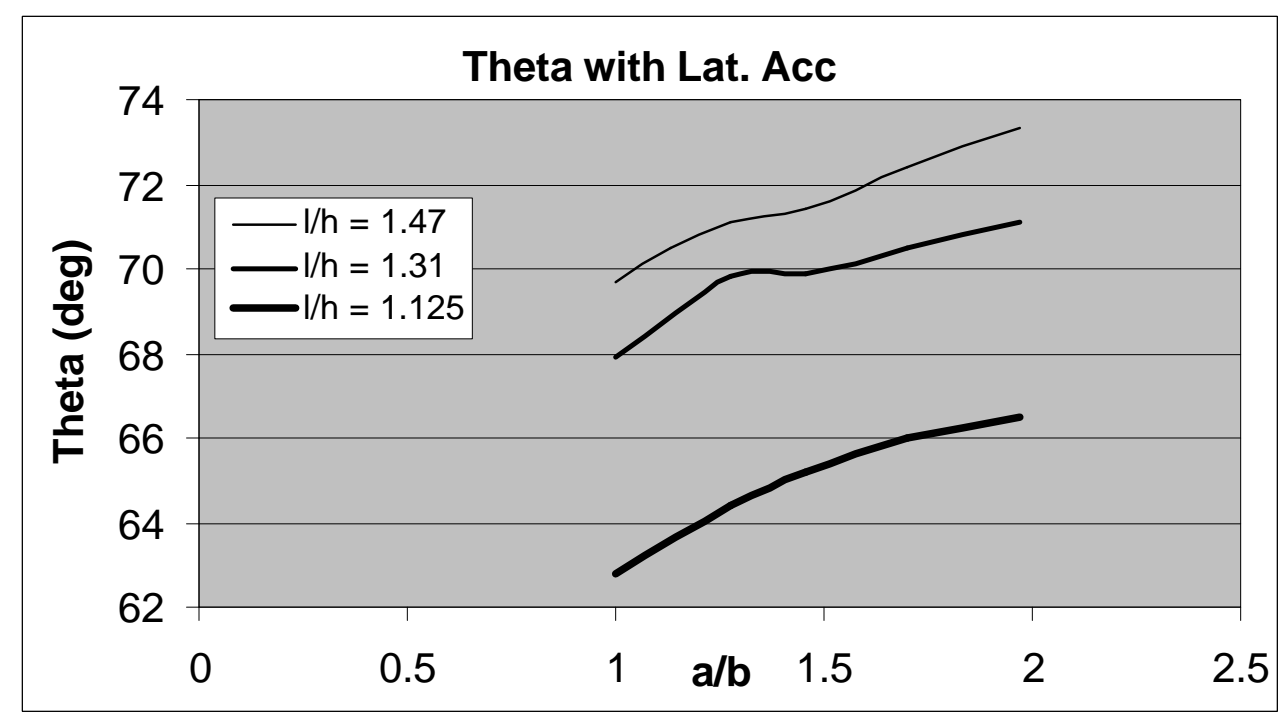

Figure 5.4: Critical Angle of Swing that will cause Liftoff but not Rollover at Different Lateral Acceleration (System is Initially at Rest)

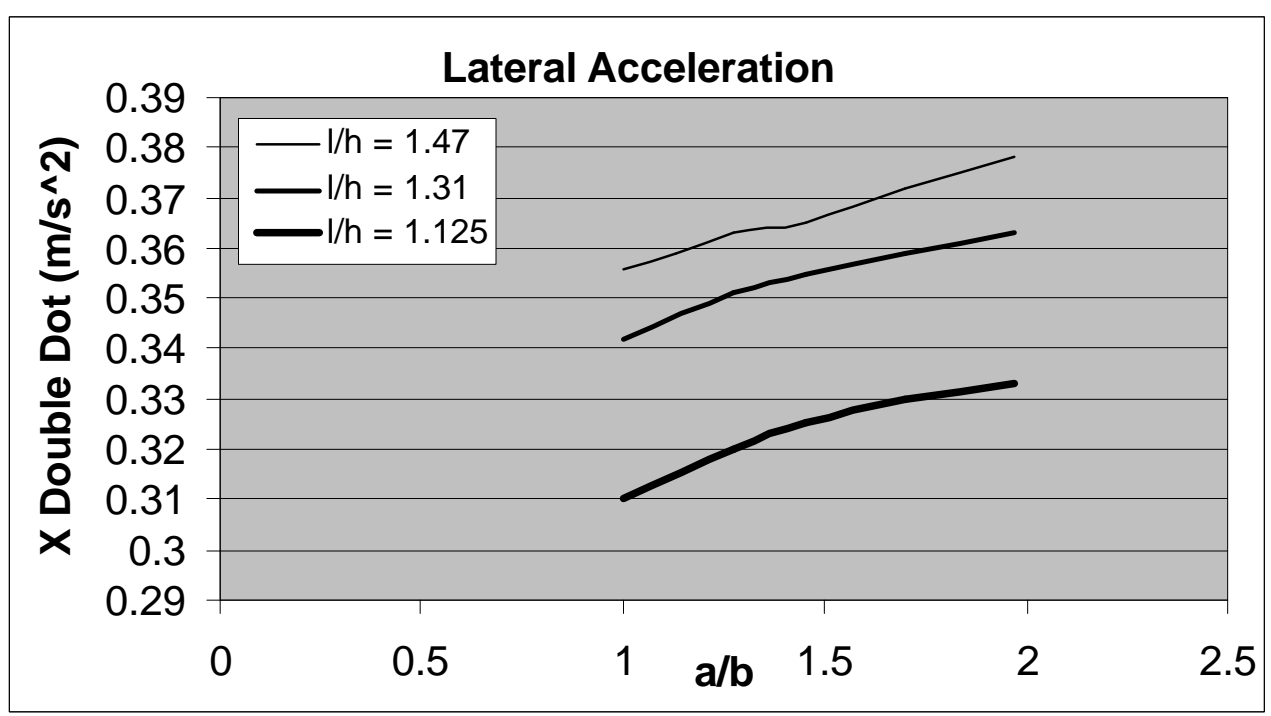

Figure 5.5: Critical Lateral Acceleration that will cause Liftoff but not Rollover (System is Initially at Rest) 


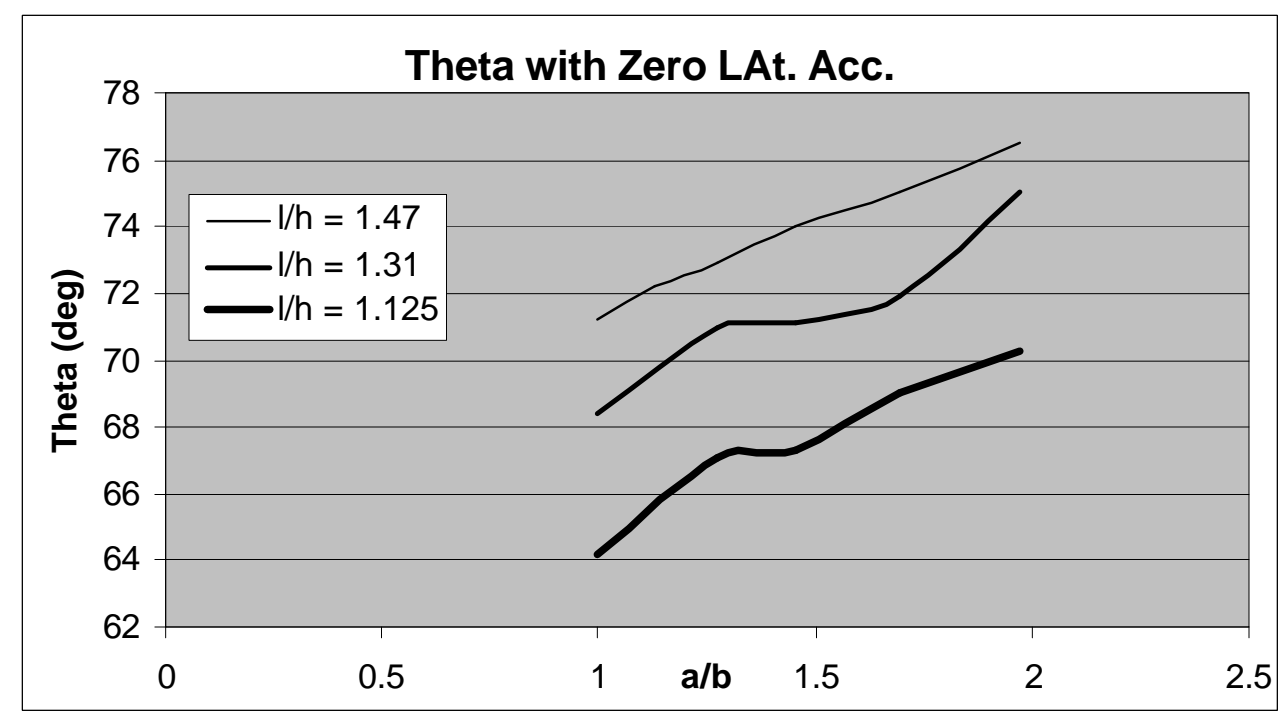

Figure 5.6: Critical Angle of Swing that will cause Liftoff but not Rollover (Zero Lateral Acceleration)

A trial and error calculation showed that a system with zero lateral acceleration starts liftoff at an angle of swing equal to 193.5 degrees $\left(\mathrm{a}=50.93_{\mathrm{cm}}, \mathrm{b}=25.87_{\mathrm{cm}}, \mathrm{h}=90_{\mathrm{cm}}\right.$, and $\mathrm{l}=$ $60.95_{\mathrm{cm}}$ ). When the pendulum is at rest (in the vertical direction), the system starts liftoff with a minimum lateral acceleration of $0.378 \mathrm{~g}$ (pendulum arm swings $\mathrm{cw} 73.3$ degrees from the vertical axis). Figures 5.7 shows the critical angle of swing for system with zero lateral acceleration. Figure 5.8 shows the angle of swing (measured $\mathrm{cw}$ form the vertical axis) changing with respect to the lateral acceleration. 


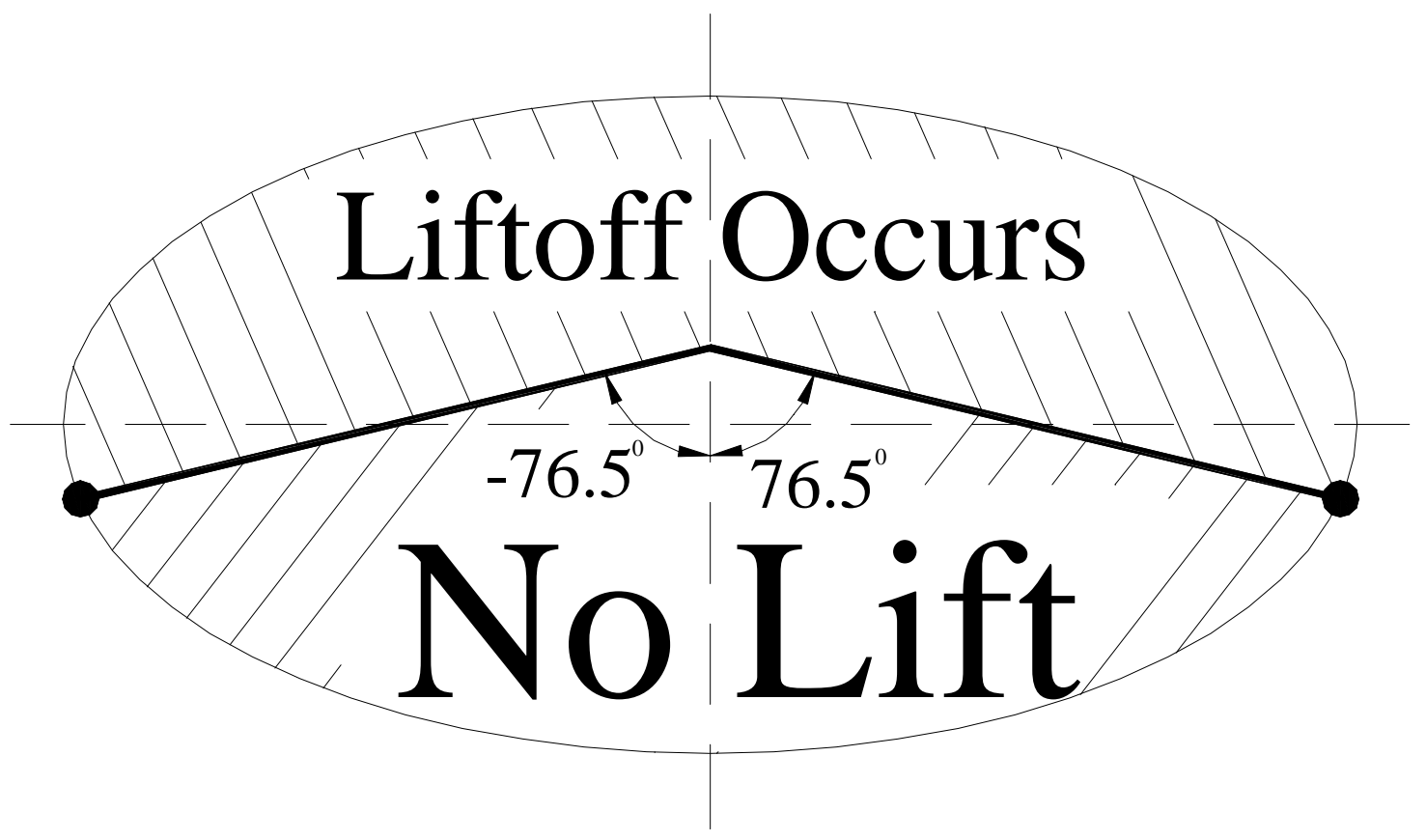

Figure 5.7: Critical Angle of Swing for Pendulum with Zero Lateral Acceleration.

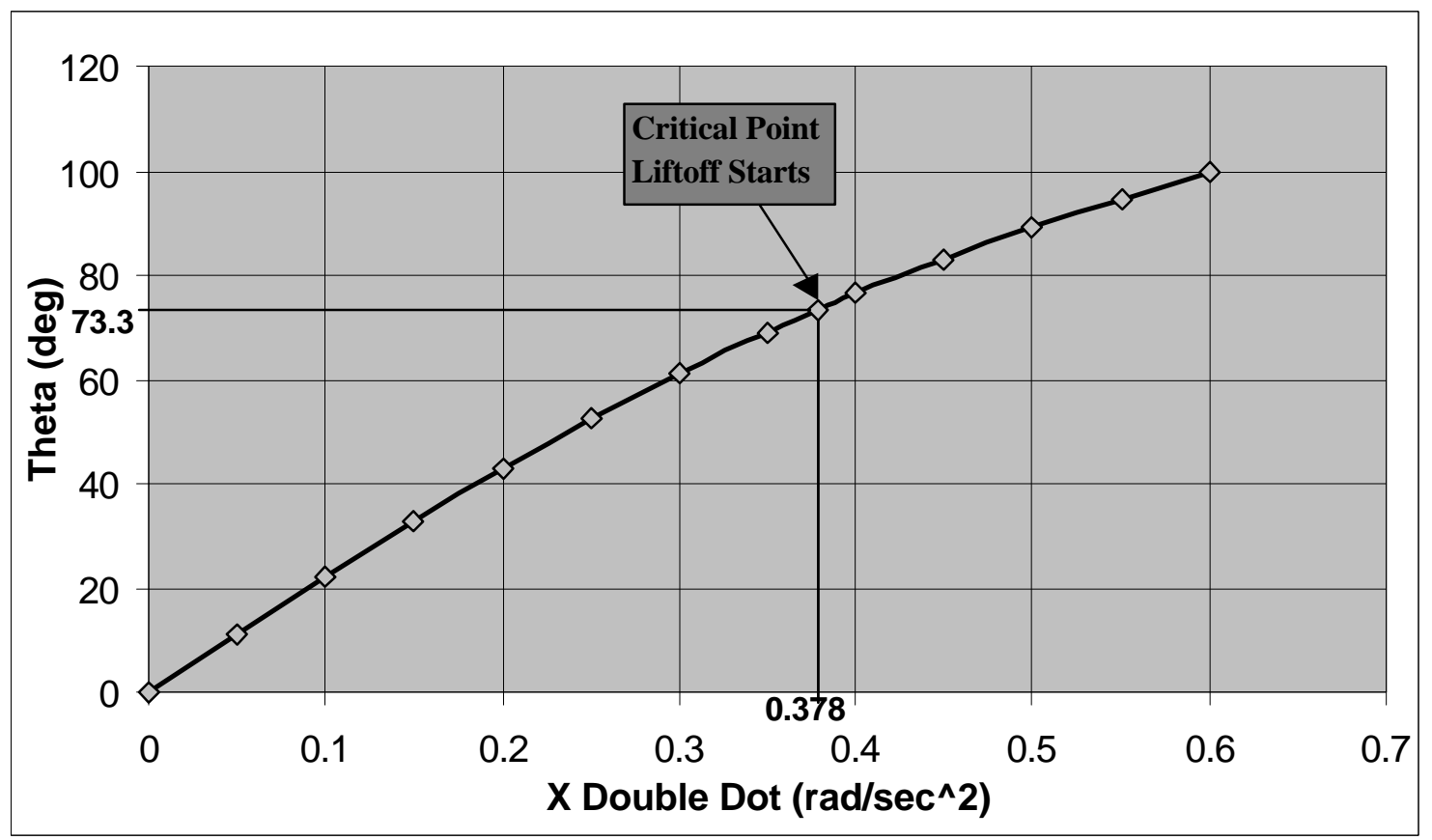

Figure 5.8: Angle of Swing vs Horizontal Lateral Acceleration for System with initial Lateral Acceleration. 


\subsection{Summary and Conclusions}

The results of this research indicate that the study of stability of the system in question requires the use of unilateral constraints to allow for support liftoff. The angular displacements, angular velocities and angular accelerations for the pendulum and support base were calculated and plotted with respect to time, for a system with fixed or lifting (unilateral) supports. The reactions at the fixed supports were also calculated and plotted with respect to time.

A mode of instability was found that allowed the base to rock back and forth from one support to two supports and then to the other support. Plots were produced to describe the critical initial conditions. One plot described the critical angle of swing under zero lateral acceleration that allows the tank to liftoff, another described the minimum lateral acceleration for tank liftoff when the pendulum is at rest.

Based on the results obtained in this research, the following conclusions can be drawn:

- Unilateral supports must be considered in order for the elliptical pendulum to be effective in simulating the sloshing dynamics of elliptical tankers.

- Different sets of equations of motion have to be considered when the system changes from one to two degrees of freedom.

- The system's stability depends on its geometric parameters, and the initial angle of the pendulum and the lateral horizontal acceleration.

- When either ratio $\mathrm{a} / \mathrm{b}$, or $1 / \mathrm{h}$ increases in magnitude, the critical lateral acceleration $\ddot{x}$ increases along with the angle of swing $\theta$. 
This research concentrated on the dynamic behavior of elliptical pendulum with unilateral supports. The simulation of the fluid mass by the elliptical trammel pendulum is an area that deserves more attention than it has received in this study. This study ignored the fact that a portion of the fluid inside the container sloshes while the rest remains fixed to the frame of the tank, and that the center of gravity at the end of the pendulum can rotate around the pendulum arm. The author hopes that this study would contribute to the field of studying stability of partially filled tank vehicles. 


\section{BIBLIOGRAPHY}

1. Ranganathan, R., Rakheja, S., and Sankar, S., "Kineto-Static Roll Plane Analysis of Articulated Tank Vehicles with Arbitrary Tank Geometry," Int. J. of Vehicle Design Vol. 10, No. 1, 1989.

2. Rakheja, S., Sankar, S., and Ranganathan, R., "Influence of Tank Design Factors on the Rollover Threshold of Partially Filled Tank Vehicles," SAE Technical Paper Series, No. 892480. Truck and Bus Meeting and Exposition, Charlotte, North Carolina, November 1989.

3. Bauer, H.F., "Dynamic Behavior of an Elastic Separating Wall in Vehicle Containers: Part I," Int. J. of Vehicle Design, Vol. 2., No. 1,1981.

4. Zhanqi, W., Rakheja, S., and Cunzhen, S., "Influence of Partition Location on the Braking Performance of a Partially-Filled Tank Truck," SAE Technical Paper Series, No. 952639, 1995.

5. Sankar, S., Sankar, T. S., and Popov, G., "Dynamics of Liquid Sloshing in Elliptical Road Containers," CSME, 1992.

6. Billing, J. R., Ranganathan, R., and Rakheja, S., "Field Testing of a Tank Truck and Study of Fluid Slosh," SAE Technical Paper Series, No. 912679. SAE International Truck and Bus Meeting, Chicago, IL, November 1991.

7. Ranganathan, R., Rakheja, S., and Sankar, S., "Influence of Liquid Load Shift on the Dynamic Response of Articulated Tank Vehicles," Vehicle System Dynamics, Vol. 19, No.4, 1990.

8. Rakheja, S., and Ranganathan, R., "Estimation of the Rollover Threshold of Heavy Vehicles Carrying Liquid Cargo: a Simplified Approach," Heavy Vehicle Systems, Int. J. of Vehicle Design, Vol. 1, No. 1, 1993.

9. Ying, Y., Miles, J.B., and Ranganathan, R., "Development of a Mechanical Analogy Model to Predict the Dynamic Behavior of Liquids in Partially Filled Tank Vehicles," SAE Technical Paper Series, No. 972307, 1997.

10. Popov, G., Sankar, S., and Vatistas, G. H., "Dynamics of Liquid Sloshing in Horizontal Cylindrical Road Containers," Proc. Inst. of Mechanical Engineers ImechE. Vol 207, Part C: Journal of Mechanical Engineering Science pp. 399-406 1993.

11. Zefran, M., and Kumar, V., "Optimal Control of Systems with Unilateral Constraints," IEEE International Conference on Robotics and Automation, May 21-27, 1998, Nagoya, Aichi, Japan. 
12. Pfeiffer, F., "Robots with Unilateral Constraints," Annual Reviews in Control Vol. 22, 1998.

13. Wosle, M., and Pfeiffer, F., "Dynamics of Multibody Systems Containing Dependent Unilateral Constraint with Friction," Journal of Vibration and Control, Vol. 2, 1996.

14. Gautam, M., Mucino, V., Salem, M., Saunders, E., and Aquaro, M., “Automotive Stability of Heavy-Duty Truck Tractor-Tanker Combinations," Final Report, Frederick Manufacturing Division of SFA, March 10, 1999.

15. Salem, M., Ph.D. Dissertation Proposal, WVU 1999.

16. Artoboleusky I., I., MECHANISMS IN MODERN ENGINEERING DESIGN, Vol. II, Mir Publishers, Moscow, 1976. 
APPENDIX 


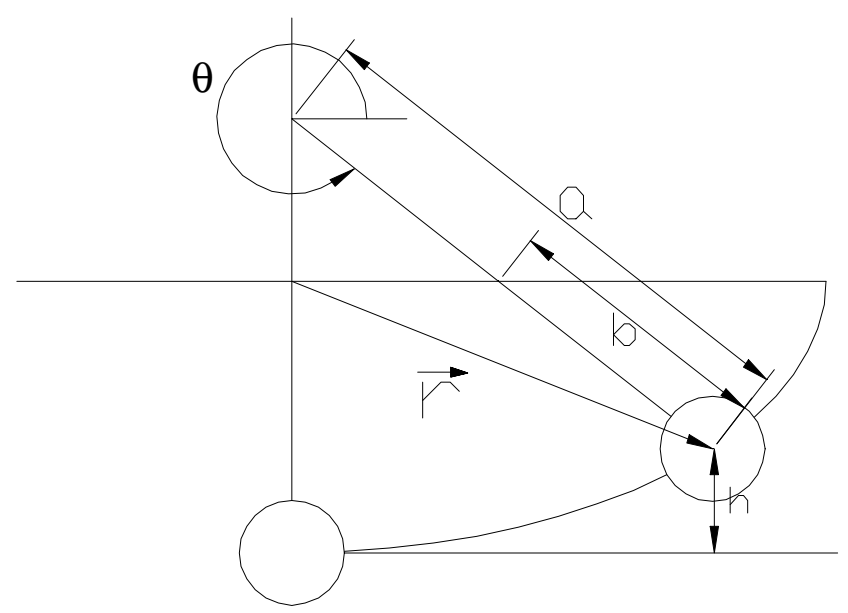

Figure A-1: Pendulum at an Arbitrary Angle Theta.

The displacement equation of the system is:

$$
\vec{r}=a \cdot \cos \theta \cdot \hat{i}+b \cdot \sin \theta \cdot \hat{j}
$$

The velocity equation is then:

$$
\vec{r}=-a \cdot \dot{\theta} \cdot \sin \theta \cdot \hat{i}+b \cdot \dot{\theta} \cdot \cos \theta \cdot \hat{j}
$$

We know that the kinetic energy for a system is:

$$
\begin{aligned}
& T=1 / 2 m|\dot{r}|^{2} \\
& |\dot{r}|^{2}=\dot{\theta}^{2}\left[a^{2} \sin ^{2} \theta+b^{2} \cos ^{2} \theta\right] \\
& \Rightarrow T=1 / 2 m \dot{\theta}^{2}\left[a^{2} \sin ^{2} \theta+b^{2} \cos ^{2} \theta\right]
\end{aligned}
$$

and that the potential energy is:

$$
\begin{aligned}
& V=m g h \\
& h=b(1+\sin \theta) \\
& \Rightarrow V=m g b(1+\sin \theta)
\end{aligned}
$$


The Lagrange equation is:

$$
\begin{aligned}
& L=T-V \\
& L=1 / 2 m \dot{\theta}^{2}\left[a^{2} \sin ^{2} \theta+b^{2} \cos ^{2} \theta\right]-m g b(1+\sin \theta)
\end{aligned}
$$

$\frac{\partial L}{\partial \dot{\theta}}=m \dot{\theta}\left[a^{2} \sin ^{2} \theta+b^{2} \cos ^{2} \theta\right]$

$\frac{d}{d t}\left(\frac{\partial L}{\partial \dot{\theta}}\right)=m \ddot{\theta}\left[a^{2} \sin ^{2} \theta+b^{2} \cos ^{2} \theta\right]+m \dot{\theta}\left[2 a^{2} \cos \theta \sin \theta \cdot \dot{\theta}-2 b^{2} \cos \theta \sin \theta \cdot \dot{\theta}\right]$

$\frac{d}{d t}\left(\frac{\partial L}{\partial \dot{\theta}}\right)=m \ddot{\theta}\left[a^{2} \sin ^{2} \theta+b^{2} \cos ^{2} \theta\right]+m \dot{\theta}^{2}\left[a^{2} \sin (2 \theta)-b^{2} \sin (2 \theta)\right]$

$\frac{d}{d t}\left(\frac{\partial L}{\partial \dot{\theta}}\right)=m \ddot{\theta}\left[a^{2} \sin ^{2} \theta+b^{2} \cos ^{2} \theta\right]+m \dot{\theta}^{2}\left(a^{2}-b^{2}\right) \sin (2 \theta)$

$\frac{\partial L}{\partial \theta}=1 / 2 m \dot{\theta}^{2}\left[2 a^{2} \cos \theta \sin \theta-2 b^{2} \cos \theta \sin \theta\right]-m g b \cos \theta$

$\frac{\partial L}{\partial \theta}=1 / 2 m \dot{\theta}^{2}\left[a^{2} \sin (2 \theta)-b^{2} \sin (2 \theta)\right]-m g b \cos \theta$

$\frac{\partial L}{\partial \theta}=1 / 2 m \dot{\theta}^{2}\left(a^{2}-b^{2}\right) \sin (2 \theta)-m g b \cos \theta$

But we also know that the Lagrange equation with no damping is:

$$
\begin{gathered}
\frac{d}{d t}\left(\frac{\partial L}{\partial \dot{\theta}}\right)-\frac{\partial L}{\partial \theta}=0 \\
\Rightarrow \ddot{\theta}\left(a^{2} \sin ^{2} \theta+b^{2} \cos ^{2} \theta\right)+\dot{\theta}^{2}\left(a^{2}-b^{2}\right) \sin (2 \theta)-\frac{1}{2} \dot{\theta}^{2}\left(a^{2}-b^{2}\right) \sin (2 \theta)+g b \cos \theta=0
\end{gathered}
$$$$
\ddot{\theta}\left(a^{2} \sin ^{2} \theta+b^{2} \cos ^{2} \theta\right)+\frac{1}{2} \ddot{\theta}^{2}\left(a^{2}-b^{2}\right) \sin 2 \theta+g b \cos \theta=0
$$ 


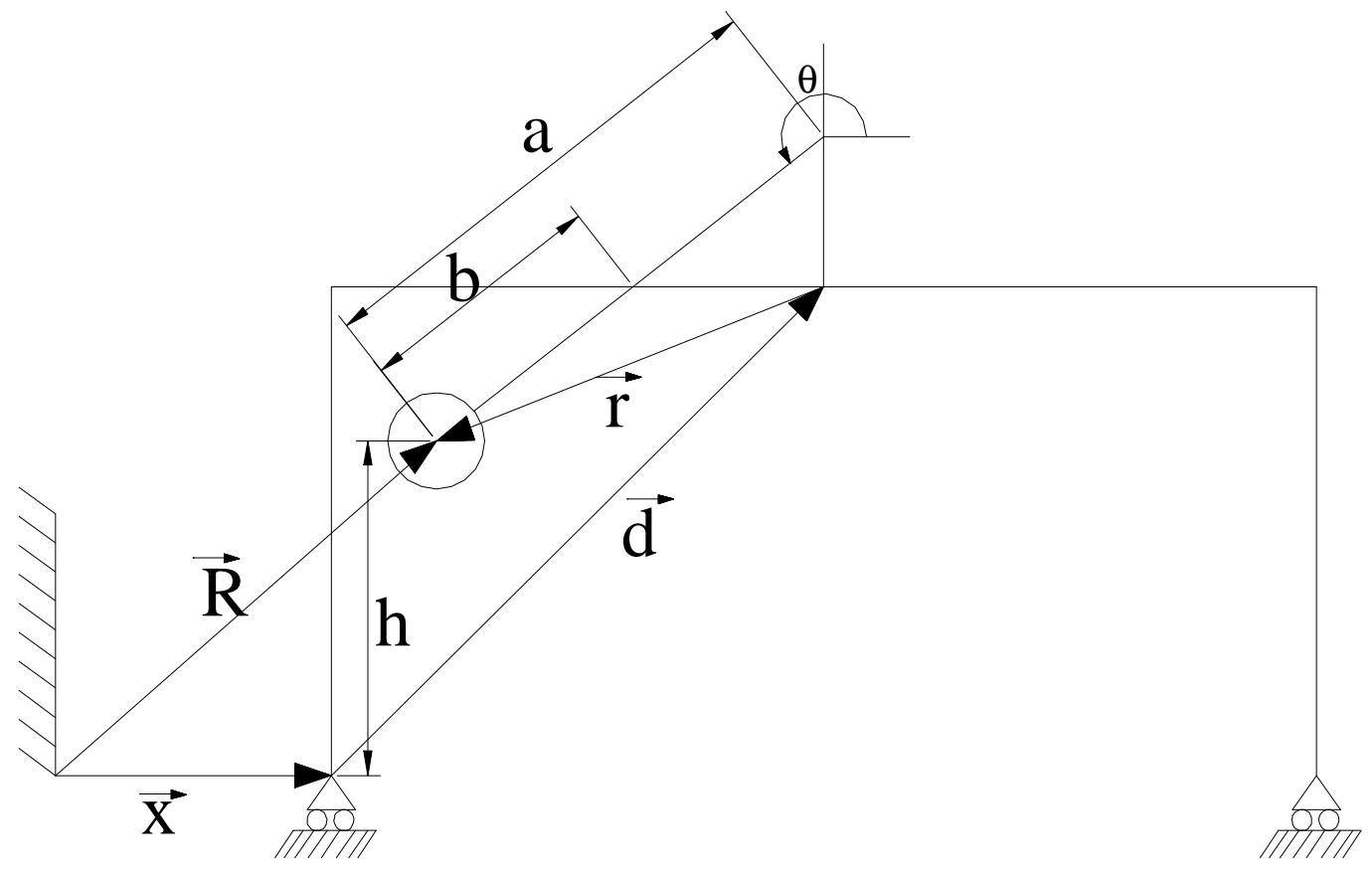

Figure A-2: System with Horizontal Lateral Acceleration.

$$
\begin{aligned}
& \vec{R}=(x+l+a \cos \theta) \hat{i}+(h+b \sin \theta) \hat{j} \\
& \dot{R}=(\dot{x}-a \dot{\theta} \sin \theta) \hat{i}+b \dot{\theta} \cos \theta \hat{j} \\
& \dot{R}^{2}=\dot{x}^{2}-2 a \dot{x} \dot{\theta} \sin \theta+\dot{\theta}^{2}\left(a^{2} \sin ^{2} \theta+b^{2} \cos ^{2} \theta\right) \\
& T=\frac{1}{2} m\left[\dot{x}^{2}-2 a \dot{x} \dot{\theta} \sin \theta+\dot{\theta}^{2}\left(a^{2} \sin ^{2} \theta+b^{2} \cos ^{2} \theta\right)\right] \\
& V=m g(h+b \sin \theta) \\
& L=\frac{1}{2} m\left[\dot{x}^{2}-2 a \dot{x} \dot{\theta} \sin \theta+\dot{\theta}^{2}\left(a^{2} \sin ^{2} \theta+b^{2} \cos ^{2} \theta\right)\right]-m g(h+b \sin \theta)
\end{aligned}
$$

We know that: $\frac{d}{d t}\left(\frac{\partial L}{\partial \dot{\theta}}\right)-\frac{\partial L}{\partial \theta}=0$ 
From the above two equations we get:

$$
\begin{aligned}
& \frac{\partial L}{\partial \dot{\theta}}=\frac{1}{2} m\left[-2 a \dot{x} \sin \theta+2 \dot{\theta}\left(a^{2} \sin ^{2} \theta+b^{2} \cos ^{2} \theta\right)\right] \\
& \frac{\partial}{\partial t}\left(\frac{\partial L}{\partial \dot{\theta}}\right)=m\left[\ddot{\theta}\left(a^{2} \sin ^{2} \theta+b^{2} \cos ^{2} \theta\right)+\dot{\theta}^{2}\left(a^{2}-b^{2}\right) \sin 2 \theta-a \ddot{x} \sin \theta-a \dot{x} \dot{\theta} \cos \theta\right] \\
& \frac{\partial L}{\partial \theta}=\frac{1}{2} m\left[\dot{\theta}^{2}\left(a^{2}-b^{2}\right) \sin 2 \theta\right]-m g b \cos \theta
\end{aligned}
$$

The equation of motion for the elliptical pendulum due to the external horizontal acceleration $\ddot{x}$ becomes:

$$
\ddot{\theta}\left(a^{2} \sin ^{2} \theta+b^{2} \cos ^{2} \theta\right)+\frac{1}{2} \dot{\theta}^{2}\left(a^{2}-b^{2}\right) \sin 2 \theta+g b \cos \theta-a \ddot{x} \sin \theta=0
$$




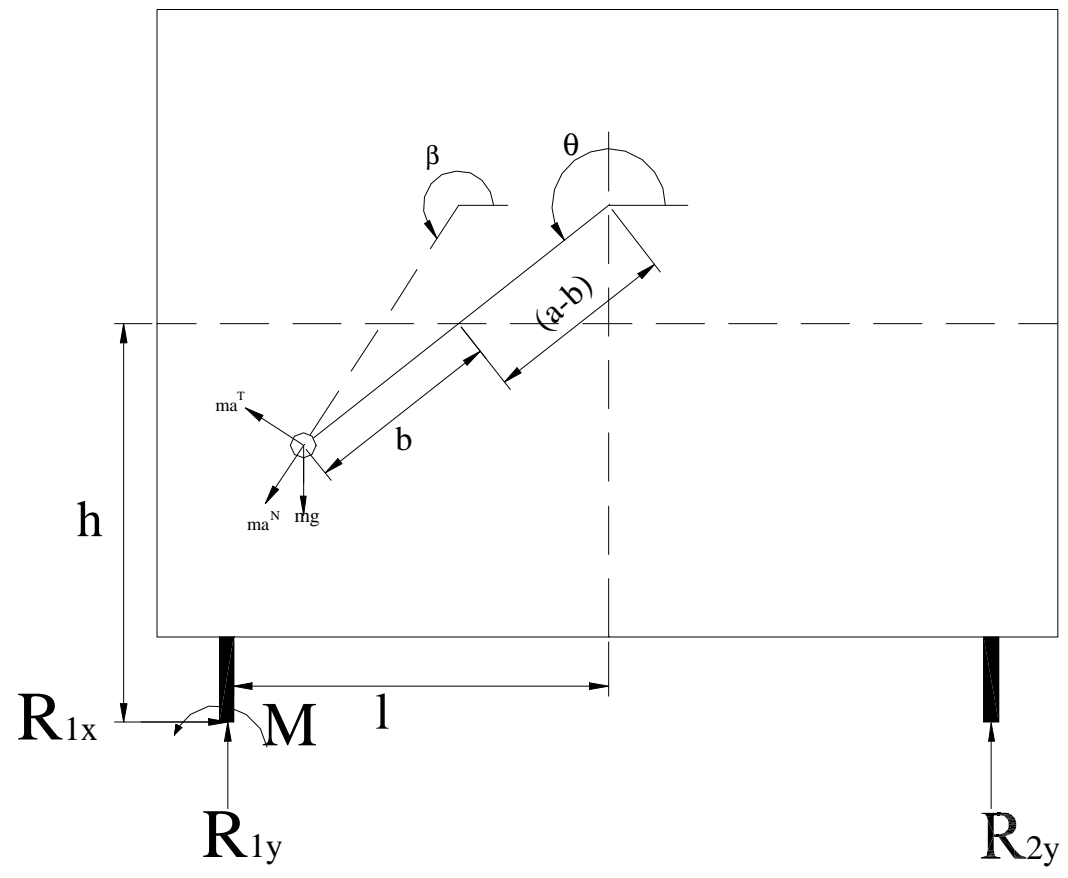

Figure A-3: Reactions at the Supports.

From the geometry of the figure we know the following:

$$
\begin{aligned}
& \sin \beta=\frac{a}{r} \sin \theta \\
& \cos \beta=\frac{b}{r} \cos \theta \\
& a^{N}=r \dot{\theta} \\
& a^{T}=r \ddot{\theta}
\end{aligned}
$$


By taking the free body diagram (FBD) we get:

$\sum F_{x}=0$

$$
\begin{aligned}
& R_{1 x}+m a^{N} \cos \beta-m a^{T} \cos (\beta-90)=0 \\
& R_{1 x}+m\left[b \dot{\theta}^{2} \cos \theta-a \ddot{\theta} \sin \theta\right]=0 \\
& \Rightarrow R_{1 x}=-m\left[b \dot{\theta}^{2} \cos \theta-a \ddot{\theta} \sin \theta\right]
\end{aligned}
$$

$\sum F_{y}=0$

$$
\begin{aligned}
& R_{1 y}+R_{2 y}-m g+m a^{N} \sin \beta+m a^{T} \sin (\beta-90)=0 \\
& R_{1 y}+R_{2 y}-m\left[g-a \dot{\theta}^{2} \sin \theta-b \ddot{\theta} \cos \theta\right]=0 \\
& \Rightarrow R_{1 y}+R_{2 y}=m\left[g-a \dot{\theta}^{2} \sin \theta-b \ddot{\theta} \cos \theta\right]
\end{aligned}
$$

$$
\sum M=0
$$

$2 l R_{2 y}-(l+a \cos \theta)\left[-m g-m a^{N} \sin \beta-m a^{T} \sin (\beta-90)\right]+$ $(h+b \sin \theta)\left[-m a^{N} \cos \beta+m a^{T} \cos (\beta-90)\right]=0$

$\Rightarrow R_{2 y}=\frac{m\left[(l+a \cos \theta)\left(g-a \dot{\theta}^{2} \sin \theta-\ddot{b} \ddot{\theta} \cos \theta\right)+(h+b \sin \theta)\left(b \dot{\theta}^{2} \cos \theta-a \ddot{\theta} \sin \theta\right)\right]}{2 l}$ 


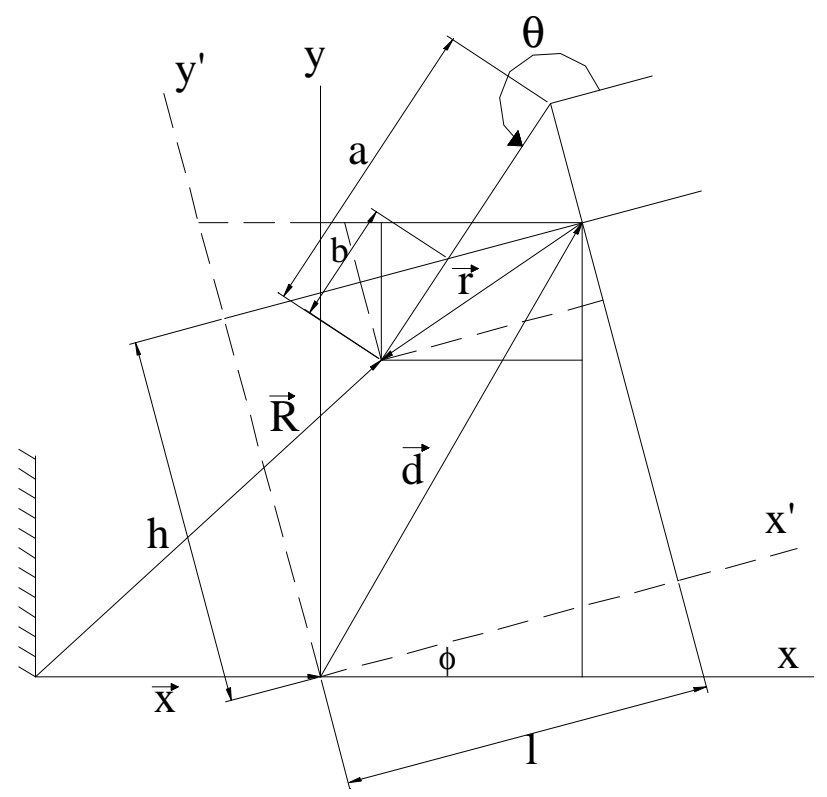

Figure A-4: Unilateral Support at $\mathrm{R}_{1 \mathrm{y}}$ only ( $\phi$ is positive $\mathrm{ccw}$ )

The displacement equation $\mathrm{R}$ for the above figure is:

$$
\begin{aligned}
& \vec{R}=\vec{x}+\vec{d}+\vec{r} \\
& \vec{x}=x \hat{i}+0 \hat{j} \\
& \vec{d}=(l-h \tan \phi) \cos \phi \hat{i}+(h+l \tan \phi) \cos \phi \vec{j} \\
& \vec{r}=(a \cos \theta-b \sin \theta \tan \phi) \cos \phi \hat{i}+(b \sin \theta+a \cos \theta \tan \phi) \cos \phi \vec{j} \\
& \Rightarrow \vec{R}=[x+(l+a \cos \theta) \cos \phi-(h+b \sin \theta) \sin \phi] \hat{i}+[(h+b \sin \theta) \cos \phi+(l+a \cos \theta) \sin \phi] \vec{j} \\
& \begin{aligned}
\frac{\partial R}{\partial t}= & \vec{R}=\quad[\dot{x}-\{(a \sin \theta \cos \phi+b \cos \theta \sin \phi) \dot{\theta}+[(l+a \cos \theta) \sin \phi+(h+b \sin \theta) \cos \phi] \dot{\phi}\}] \hat{i}
\end{aligned} \\
& \quad+[(b \cos \theta \cos \phi-a \sin \theta \sin \phi) \dot{\theta}+[(l+a \cos \theta) \cos \phi-(h+b \sin \theta) \sin \phi] \dot{\phi}] \hat{j}
\end{aligned}
$$




$$
\begin{aligned}
\dot{R}^{2}= & \dot{x}^{2}-2 \dot{x} \dot{\theta}(a \sin \theta \cos \phi+b \cos \theta \sin \phi)-2 \dot{x} \dot{\phi}[(l+a \cos \theta) \sin \phi+(h+b \sin \theta) \cos \phi] \\
& +\dot{\theta}^{2}\left(a^{2} \sin ^{2} \theta+b^{2} \cos ^{2} \theta\right)+2 \dot{\theta} \dot{\phi}(a h \sin \theta+b l \cos \theta+a b) \\
& +\dot{\phi}^{2}\left[(h+a \cos \theta)^{2}+(l+b \sin \theta)^{2}\right]
\end{aligned}
$$

The kinetic energy equation is such that:

$$
\begin{gathered}
T=1 / 2 m \dot{R}^{2} \\
\Rightarrow T=1 / 2 m\left[\begin{array}{c}
\dot{x}^{2}-2 \dot{x} \dot{\theta}(a \sin \theta \cos \phi+b \cos \theta \sin \phi)-2 \dot{x} \dot{\phi}[(l+a \cos \theta) \sin \phi+(h+b \sin \theta) \cos \phi] \\
+\dot{\theta}^{2}\left(a^{2} \sin ^{2} \theta+b^{2} \cos ^{2} \theta\right)+2 \dot{\theta} \dot{\phi}(a h \sin \theta+b l \cos \theta+a b) \\
+\dot{\phi}^{2}\left[(l+a \cos \theta)^{2}+(h+b \sin \theta)^{2}\right]
\end{array}\right]
\end{gathered}
$$

And the potential energy equation is:

$$
V=m g[(h+b \sin \theta) \cos \phi+(l+a \cos \theta) \sin \phi]
$$

The Lagrange equation is:

$$
\mathrm{L}=\mathrm{T}-\mathrm{V}
$$

Differentiating the Lagrange equation with respect to $\dot{\theta}$ and then differentiating this last equation with respect to time we will get the following:

$$
\begin{gathered}
\frac{\partial L}{\partial \dot{\theta}}=m\left[\dot{\theta}\left(a^{2} \sin ^{2} \theta+b^{2} \cos ^{2} \theta\right)+\dot{\phi}(a h \sin \theta+b l \cos \theta+a b)-\dot{x}(a \sin \theta \cos \phi+b \cos \theta \sin \phi)\right] \\
\frac{\partial}{\partial t}\left(\frac{\partial L}{\partial \dot{\theta}}\right)=m\left[\begin{array}{l}
\ddot{\theta}\left(a^{2} \sin ^{2} \theta+b^{2} \cos ^{2} \theta\right)+\dot{\theta}^{2}\left(a^{2}-b^{2}\right) \sin 2 \theta+\ddot{\phi}(a h \sin \theta+b l \cos \theta+a b) \\
+\dot{\theta} \dot{\phi}(a h \cos \theta-b l \sin \theta)-\ddot{x}(a \sin \theta \cos \phi+b \cos \theta \sin \phi) \\
\dot{x} \dot{\theta}(a \cos \theta \cos \phi-b \sin \theta \sin \phi)-\dot{x} \dot{\phi}(b \cos \theta \cos \phi-a \sin \theta \sin \phi)
\end{array}\right]
\end{gathered}
$$

Now differentiating the Lagrange equation with respect to $\theta$ will give us: 


$$
\begin{aligned}
\frac{\partial L}{\partial \theta}=1 / 2 m\left[\begin{array}{l}
-2 \dot{x} \dot{\theta}(a \cos \theta \cos \phi-b \sin \theta \sin \phi)-2 \dot{x} \dot{\phi}(-a \sin \theta \sin \phi+b \cos \theta \cos \phi)+\dot{\theta}^{2}\left(a^{2}-b^{2}\right) \sin 2 \theta \\
+2 \dot{\theta} \dot{\phi}(a h \cos \theta-b l \sin \theta)+2 \dot{\phi}^{2}\left[-a l \sin \theta+b h \cos \theta-\frac{1}{2}\left(a^{2}-b^{2}\right) \sin 2 \theta\right]
\end{array}\right] \\
\quad-m g[b \cos \theta \cos \phi-a \sin \theta \sin \phi]
\end{aligned}
$$

We know that:

$$
\frac{\partial}{\partial t}\left(\frac{\partial L}{\partial \dot{\theta}}\right)-\frac{\partial L}{\partial \theta}=0
$$

$$
\begin{aligned}
\Rightarrow & \ddot{\theta}\left(a^{2} \sin ^{2} \theta+b^{2} \cos ^{2} \theta\right)+1 / 2 \dot{\theta}^{2}\left(a^{2}-b^{2}\right) \sin 2 \theta+\ddot{\phi}(a h \sin \theta+b l \cos \theta+a b) \\
& +\dot{\phi}^{2}\left[a l \sin \theta-b h \cos \theta+\frac{1}{2}\left(a^{2}-b^{2}\right) \sin 2 \theta\right]+g(b \cos \theta \cos \phi-a \sin \theta \sin \phi) \\
& \quad \ddot{x}(a \sin \theta \cos \phi+b \cos \theta \sin \phi)=0
\end{aligned}
$$

By differentiating the Lagrange equation with respect to $\dot{\phi}$ and then with respect to time we get the following:

$$
\frac{\partial L}{\partial \dot{\phi}}=1 / 2 m\left[\begin{array}{l}
-2 \dot{x}[(l+a \cos \theta) \sin \phi+(h+b \sin \theta) \cos \phi]+2 \dot{\theta}(a h \sin \theta+b l \cos \theta+a b) \\
+2 \dot{\phi}\left[(l+a \cos \theta)^{2}+(h+b \sin \theta)^{2}\right]
\end{array}\right]
$$

$$
\frac{\partial}{\partial t}\left(\frac{\partial L}{\partial \dot{\phi}}\right)=m\left[\begin{array}{l}
-\ddot{x}[(l+a \cos \theta) \sin \phi+(h+b \sin \theta) \cos \phi]+\dot{x} \dot{\theta}[a \sin \theta \sin \phi-b \cos \theta \cos \phi] \\
-\dot{x} \dot{\phi}[(l+a \cos \theta) \cos \phi-(h+b \sin \theta) \sin \phi]+\ddot{\theta}(a h \sin \theta+b l \cos \theta+a b) \\
\dot{\ddot{\theta}}^{2}(a h \cos \theta-b l \sin \theta)+\ddot{\phi}\left[(l+a \cos \theta)^{2}+(h+b \sin \theta)^{2}\right] \\
-2 \dot{\theta} \dot{\phi}\left[a l \sin \theta-b h \cos \theta+\frac{1}{2}\left(a^{2}-b^{2}\right) \sin 2 \theta\right]
\end{array}\right]
$$


Now differentiating the Lagrange equation with respect to $\phi$ gives us the following:

$$
\begin{aligned}
\frac{\partial L}{\partial \phi}= & \frac{1}{2} m[2 \dot{x} \dot{\theta}[a \sin \theta \sin \phi-b \cos \theta \cos \phi]-2 \dot{x} \dot{\phi}[(l+a \cos \theta) \cos \phi-(h+b \sin \theta) \sin \phi]] \\
& -m g[-(h+b \sin \theta) \sin \phi+(l+a \cos \theta) \cos \phi]
\end{aligned}
$$

But we know that:

$$
\frac{\partial}{\partial t}\left(\frac{\partial L}{\partial \dot{\phi}}\right)-\frac{\partial L}{\partial \phi}=0
$$

\footnotetext{
$\Rightarrow \ddot{\theta}(a h \sin \theta+b l \cos \theta+a b)+\dot{\theta}^{2}(a h \cos \theta-b l \sin \theta)+\ddot{\phi}\left[(l+a \cos \theta)^{2}+(h+b \sin \theta)^{2}\right]$

$-2 \dot{\theta} \dot{\phi}\left[a l \sin \theta-b h \cos \theta+\frac{1}{2}\left(a^{2}-b^{2}\right) \sin 2 \theta\right]+g[(l+a \cos \theta) \cos \phi-(h+b \sin \theta) \sin \phi]$

$-\ddot{x}[(l+a \cos \theta) \sin \phi+(h+b \sin \theta) \cos \phi]=0$
} 


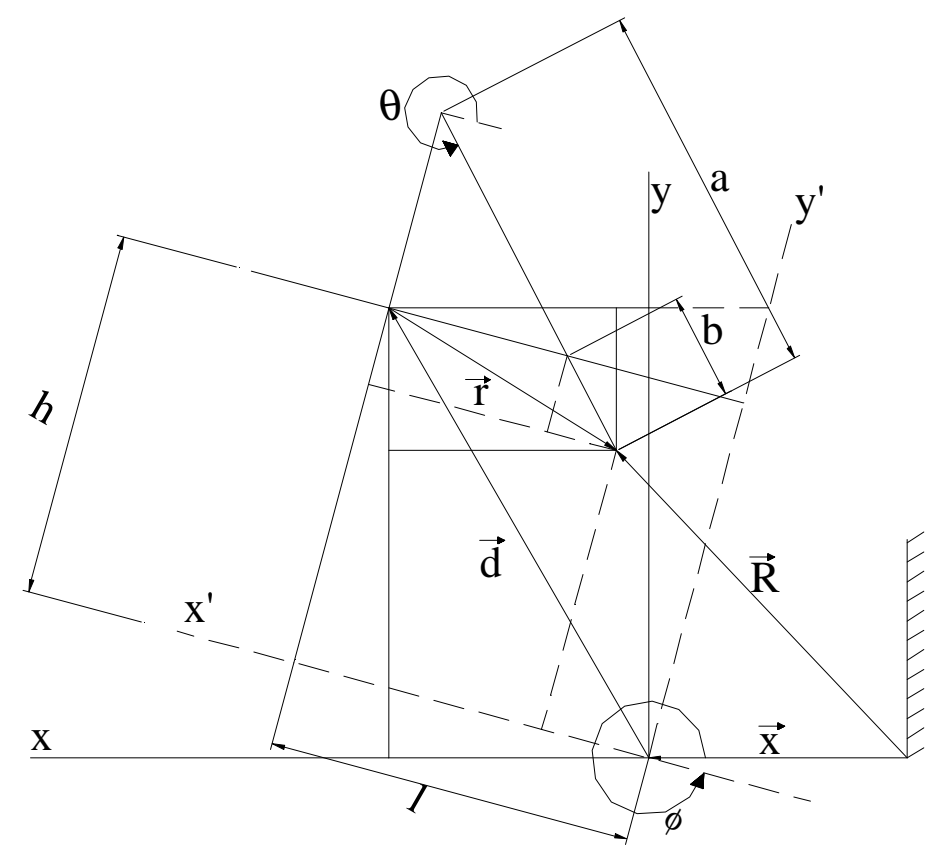

Figure A-5: Unilateral Support at $\mathrm{R}_{2 \mathrm{y}}$ only ( $\phi$ is negative $\mathrm{cw}$ )

The displacement equation $\mathrm{R}$ for the above figure is:

$$
\begin{aligned}
& \vec{R}=\vec{x}+\vec{d}+\vec{r} \\
& \vec{x}=-x \hat{i} \\
& \vec{d}=(l \cos 180-h \tan \phi) \cos \phi \hat{i}+(h-l \tan \phi) \cos \phi \vec{j} \\
& \vec{r}=(a \cos \theta-b \sin \theta \tan \phi) \cos \phi \hat{i}+(b \sin \theta+a \cos \theta \tan \phi) \cos \phi \vec{j} \\
& \Rightarrow \vec{R}=[-x-(l-a \cos \theta) \cos \phi-(h+b \sin \theta) \sin \phi] \hat{i}+[(h+b \sin \theta) \cos \phi-(l-a \cos \theta) \sin \phi] \vec{j} \\
& \begin{aligned}
\frac{\partial R}{\partial t}= & \vec{R}=\quad[-\dot{x}-\{(a \sin \theta \cos \phi+b \cos \theta \sin \phi) \dot{\theta}-[(l-a \cos \theta) \sin \phi-(h+b \sin \theta) \cos \phi] \dot{\phi}\}] \hat{i}
\end{aligned} \\
& \quad+[(b \cos \theta \cos \phi-a \sin \theta \sin \phi) \dot{\theta}-[(l-a \cos \theta) \cos \phi+(h+b \sin \theta) \sin \phi] \dot{\phi}] \hat{j}
\end{aligned}
$$




$$
\begin{aligned}
\dot{R}^{2}= & \dot{x}^{2}+2 \dot{x} \dot{\theta}(a \sin \theta \cos \phi+b \cos \theta \sin \phi)-2 \dot{x} \dot{\phi}[(l-a \cos \theta) \sin \phi+(h+b \sin \theta) \cos \phi] \\
& +\dot{\theta}^{2}\left(a^{2} \sin ^{2} \theta+b^{2} \cos ^{2} \theta\right)+2 \dot{\theta} \dot{\phi}(a h \sin \theta-b l \cos \theta+a b) \\
& +\dot{\phi}^{2}\left[(l-a \cos \theta)^{2}+(h+b \sin \theta)^{2}\right]
\end{aligned}
$$

The kinetic energy equation is such that:

$$
\begin{gathered}
T=1 / 2 m \dot{R}^{2} \\
\Rightarrow T=1 / 2 m\left[\begin{array}{l}
\left.\dot{x}^{2}+2 \dot{x} \dot{\theta}(a \sin \theta \cos \phi+b \cos \theta \sin \phi)-2 \dot{x} \dot{\phi}[(l-a \cos \theta) \sin \phi+(h+b \sin \theta) \cos \phi]\right] \\
+\dot{\theta}^{2}\left(a^{2} \sin ^{2} \theta+b^{2} \cos ^{2} \theta\right)+2 \dot{\theta} \dot{\phi}(a h \sin \theta-b l \cos \theta+a b) \\
\dot{\phi}^{2}\left[(l-a \cos \theta)^{2}+(h+b \sin \theta)^{2}\right]
\end{array}\right]
\end{gathered}
$$

And the potential energy equation is:

$$
V=m g[(h+b \sin \theta) \cos \phi-(l-a \cos \theta) \sin \phi]
$$

The Lagrange equation is:

$$
\mathrm{L}=\mathrm{T}-\mathrm{V}
$$

Differentiating the Lagrange equation with respect to $\dot{\theta}$ and then differentiating this last equation with respect to time we will get the following:

$$
\begin{gathered}
\frac{\partial L}{\partial \dot{\theta}}=m\left[\dot{\theta}\left(a^{2} \sin ^{2} \theta+b^{2} \cos ^{2} \theta\right)+\dot{\phi}(a h \sin \theta-b l \cos \theta+a b)+\dot{x}(a \sin \theta \cos \phi+b \cos \theta \sin \phi)\right] \\
\frac{\partial}{\partial t}\left(\frac{\partial L}{\partial \dot{\theta}}\right)=m\left[\begin{array}{l}
\left.\ddot{\theta}\left(a^{2} \sin ^{2} \theta+b^{2} \cos ^{2} \theta\right)+\dot{\theta}^{2}\left(a^{2}-b^{2}\right) \sin 2 \theta+\ddot{\phi}(a h \sin \theta-b l \cos \theta+a b)\right] \\
+\dot{\theta} \dot{\phi}(a h \cos \theta+b l \sin \theta)+\ddot{x}(a \sin \theta \cos \phi+b \cos \theta \sin \phi) \\
\dot{x} \dot{\theta}(a \cos \theta \cos \phi-b \sin \theta \sin \phi)+\dot{x} \dot{\phi}(b \cos \theta \cos \phi-a \sin \theta \sin \phi)
\end{array}\right]
\end{gathered}
$$

Now differentiating the Lagrange equation with respect to $\theta$ will give us: 


$$
\begin{aligned}
\frac{\partial L}{\partial \theta}=1 / 2 m\left[\begin{array}{l}
2 \dot{x} \dot{\theta}(a \cos \theta \cos \phi-b \sin \theta \sin \phi)-2 \dot{x} \dot{\phi}(a \sin \theta \sin \phi-b \cos \theta \cos \phi)+\dot{\theta}^{2}\left(a^{2}-b^{2}\right) \sin 2 \theta \\
+2 \dot{\theta} \dot{\phi}(a h \cos \theta+b l \sin \theta)-2 \dot{\phi}^{2}\left[a l \sin \theta-b h \cos \theta-\frac{1}{2}\left(a^{2}+b^{2}\right) \sin 2 \theta\right]
\end{array}\right] \\
-m g[b \cos \theta \cos \phi-a \sin \theta \sin \phi]
\end{aligned}
$$

We know that:

$$
\frac{\partial}{\partial t}\left(\frac{\partial L}{\partial \dot{\theta}}\right)-\frac{\partial L}{\partial \theta}=0
$$

$$
\begin{aligned}
\Rightarrow & \ddot{\theta}\left(a^{2} \sin ^{2} \theta+b^{2} \cos ^{2} \theta\right)+1 / 2 \dot{\theta}^{2}\left(a^{2}-b^{2}\right) \sin 2 \theta+\ddot{\phi}(a h \sin \theta-b l \cos \theta+a b) \\
& +\dot{\phi}^{2}\left[a l \sin \theta-b h \cos \theta-\frac{1}{2}\left(a^{2}+b^{2}\right) \sin 2 \theta\right]+g(b \cos \theta \cos \phi-a \sin \theta \sin \phi) \\
& +\ddot{x}(a \sin \theta \cos \phi+b \cos \theta \sin \phi)=0
\end{aligned}
$$

By differentiating the Lagrange equation with respect to $\dot{\phi}$ and then with respect to time we get the following:

$$
\frac{\partial L}{\partial \dot{\phi}}=1 / 2 m\left[\begin{array}{l}
-2 \dot{x}[(l-a \cos \theta) \sin \phi-(h+b \sin \theta) \cos \phi]+2 \dot{\theta}(a h \sin \theta-b l \cos \theta+a b) \\
+2 \dot{\phi}\left[(l-a \cos \theta)^{2}+(h+b \sin \theta)^{2}\right]
\end{array}\right]
$$

$$
\frac{\partial}{\partial t}\left(\frac{\partial L}{\partial \dot{\phi}}\right)=m\left[\begin{array}{l}
\ddot{-x}[(l-a \cos \theta) \sin \phi-(h+b \sin \theta) \cos \phi]-\dot{x} \dot{\theta}[a \sin \theta \sin \phi-b \cos \theta \cos \phi] \\
-\dot{x} \dot{\phi}[(l-a \cos \theta) \cos \phi+(h+b \sin \theta) \sin \phi]+\ddot{\theta}(a h \sin \theta-b l \cos \theta+a b) \\
\dot{\theta}^{2}(a h \cos \theta+b l \sin \theta)+\ddot{\phi}\left[(l-a \cos \theta)^{2}+(h+b \sin \theta)^{2}\right] \\
+\dot{\theta} \dot{\phi}\left[-2 a l \sin \theta+2 b h \cos \theta+\left(a^{2}+b^{2}\right) \sin 2 \theta\right]
\end{array}\right]
$$


Now differentiating the Lagrange equation with respect to $\phi$ gives us the following:

$$
\begin{aligned}
\frac{\partial L}{\partial \phi}= & \frac{1}{2} m[-\dot{2} \dot{x} \dot{\theta}[a \sin \theta \sin \phi-b \cos \theta \cos \phi]-2 \dot{x} \dot{\phi}[(l-a \cos \theta) \cos \phi+(h+b \sin \theta) \sin \phi]] \\
& -m g[-(h+b \sin \theta) \sin \phi-(l-a \cos \theta) \cos \phi]
\end{aligned}
$$

But we know that:

$$
\frac{\partial}{\partial t}\left(\frac{\partial L}{\partial \dot{\phi}}\right)-\frac{\partial L}{\partial \phi}=0
$$

$\Rightarrow \ddot{\theta}(a h \sin \theta-b l \cos \theta+a b)+\dot{\theta}^{2}(a h \cos \theta+b l \sin \theta)+\ddot{\phi}\left[(l-a \cos \theta)^{2}+(h+b \sin \theta)^{2}\right]$
$+\ddot{\theta} \dot{\phi}\left[-2 a l \sin \theta+2 b h \cos \theta+\left(a^{2}+b^{2}\right) \sin 2 \theta\right]-g[(l-a \cos \theta) \cos \phi+(h+b \sin \theta) \sin \phi]$
$-\ddot{x}[(l-a \cos \theta) \sin \phi-(h+b \sin \theta) \cos \phi]=0$


$\%$ This subprogram will find the angular displacement, velocity, and acceleration of $\%$ the Elliptical Tank with fixed support.

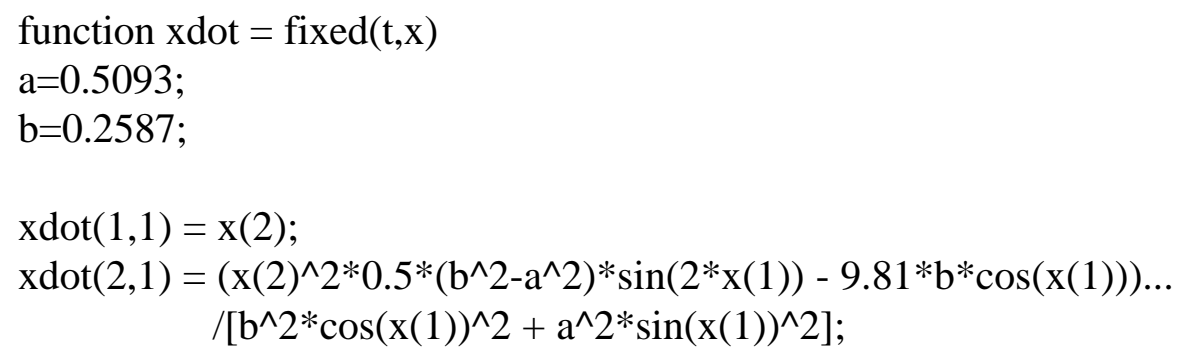

$\%$ Main Program uses the subprogram above to print the results in form of figures with $\%$ respect to time.

close all

clear

$\mathrm{tt}=\left[\begin{array}{ll}0 & 10\end{array}\right]$

\%initial value of $\mathrm{t}$

$\mathrm{x} 0=[$ pi 0$]$;

$\%$ column vector of initial condition.

$[\mathrm{t}, \mathrm{x}]=$ ode $45($ 'fixed',tt, $\mathrm{x} 0)$;

figure; $\operatorname{plot}(\mathrm{t}, \mathrm{x}(:, 1)$, 'black');

title('Angular Displacement');

ylabel('Theta (rad)'); xlabel('time (sec)'); grid;

figure; $\operatorname{plot}(\mathrm{t}, \mathrm{x}(:, 2)$, 'black');

title('Angular Velocity');

ylabel('Theta dot'); xlabel('time (sec)'); grid;

\%*******************Calculations for Theta double dot $* * * * * * * * * * * * * * * * * * * * * * *$

for $\mathrm{j}=1$ :length $(\mathrm{x}(:, 2))-1$

$\mathrm{dt}=\mathrm{t}(\mathrm{j}+1)-\mathrm{t}(\mathrm{j})$;

theta_double_dot $(\mathrm{j})=(\mathrm{x}(\mathrm{j}+1,2)-\mathrm{x}(\mathrm{j}, 2)) / \mathrm{dt}$;

end

theta_double_dot $($ length $(\mathrm{x}(:, 2)))=0$;

figure; plot(t,theta_double_dot, 'black');

title('Angular Acceleration');

ylabel('Theta double dot'); xlabel('time (sec)'); grid; 
$\%$ This subprogram will find the angular displacement, velocity, and acceleration of $\%$ the Elliptical Tank due to a known external horizontal acceleration $\ddot{x}$.

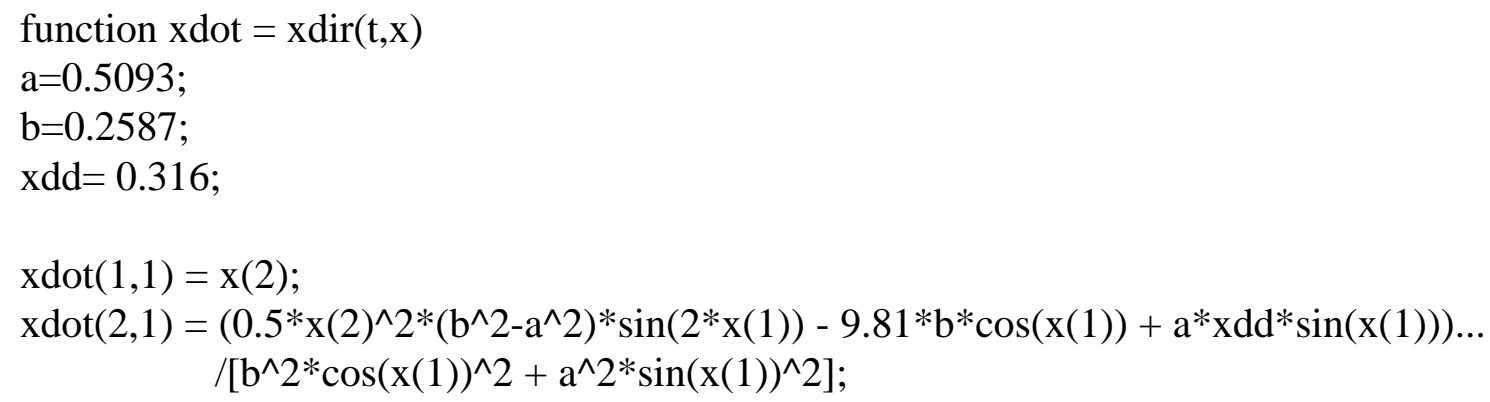

$\%$ Main Program uses the subprogram above to print the results in form of figures with $\%$ respect to time

close all

clear

$\mathrm{tt}=\left[\begin{array}{ll}0 & 10\end{array}\right]$

\%initial value of time

$\mathrm{x} 0=[\mathrm{pi} 0]$;

\%column vector of initial condition

$[\mathrm{t}, \mathrm{x}]=\operatorname{ode} 45\left({ }^{\mathrm{x} d i r}, \mathrm{tt}, \mathrm{x} 0\right)$;

figure; $\operatorname{subplot}(2,2,1), \operatorname{plot}(\mathrm{t}, \mathrm{x}(:, 1)$, 'black');

title('Angular Displacement');

ylabel('Theta $(\mathrm{rad})$ '); xlabel('time $(\mathrm{sec})$ '); grid;

$\operatorname{subplot}(2,2,2), \operatorname{plot}(\mathrm{t}, \mathrm{x}(:, 2)$, 'black');

title('Angular Velocity');

ylabel('Theta dot'); xlabel('time (sec)'); grid;

$\% * * * * * * * * * * * * * * * * * * *$ Calculations for Theta double dot $* * * * * * * * * * * * * * * * * * * * * *$

for $\mathrm{j}=1$ :length $(\mathrm{x}(:, 2))-1$

$\mathrm{dt}=\mathrm{t}(\mathrm{j}+1)-\mathrm{t}(\mathrm{j})$;

theta_double_dot $(\mathrm{j})=(\mathrm{x}(\mathrm{j}+1,2)-\mathrm{x}(\mathrm{j}, 2)) / \mathrm{dt}$;

end

theta_double_dot $($ length $(\mathrm{x}(:, 2)))=0$;

subplot(2,2,3.5),plot(t,theta_double_dot, 'black');

title('Angular Acceleration');

ylabel('Theta double dot'); xlabel('time (sec)'); grid; 
$\%$ Main Program uses the subprogram xdir to calculate the reactions at the supports $\%$ in the $\mathrm{x}$ and $\mathrm{y}$-direction, the results are printed in the form of figure respect to time

close all

clear

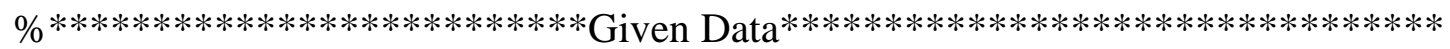

$\mathrm{a}=0.5093$

$\mathrm{b}=0.2587$;

$\mathrm{m}=11349$;

$\mathrm{l}=1.2 ; \mathrm{h}=0.6095$;

$\%$ Masses are in Kilograms

$\%$ Distance are in Meter

\%********************Calculations for Theta, and Theta $\operatorname{dot}^{*} * * * * * * * * * * * * * * * * * * * *$

$\mathrm{tt}=\left[\begin{array}{ll}0 & 10\end{array}\right]$

$\%$ initial value of time

$\mathrm{x} 0=[$ pi 0$]$;

$\%$ column vector of initial condition

$[\mathrm{t}, \mathrm{x}]=\operatorname{ode} 45\left({ }^{\mathrm{x} d i r}, \mathrm{tt}, \mathrm{x} 0\right)$;

$\% * * * * * * * * * * * * * * * * * * *$ Calculations for Theta double dot $* * * * * * * * * * * * * * * * * * * * * * *$

for $\mathrm{j}=1$ :length $(\mathrm{x}(:, 2))-1$

$\mathrm{dt}=\mathrm{t}(\mathrm{j}+1)-\mathrm{t}(\mathrm{j})$;

thetadd $(\mathrm{j})=(\mathrm{x}(\mathrm{j}+1,2)-\mathrm{x}(\mathrm{j}, 2)) / \mathrm{dt}$;

end

thetadd $($ length $(\mathrm{x}(:, 2)))=0$;

\%**************Main Calculations for the Stability of the Pendulum**************

for $\mathrm{i}=1$ :length(t)

$\mathrm{A}=\left[\begin{array}{lll}1 & 0 & 0 ; \ldots\end{array}\right.$

$011 ; \ldots$

$00(2 * 1)]$;

$\mathrm{B}=\left[-\mathrm{m}^{*}\left(\mathrm{~b}^{*} \mathrm{x}(\mathrm{i}, 2)^{\wedge} 2^{*} \cos (\mathrm{x}(\mathrm{i}, 1))-\mathrm{a}^{*} \operatorname{thetadd}(\mathrm{i}) * \sin (\mathrm{x}(\mathrm{i}, 1))\right)\right.$

$\mathrm{m}^{*}\left(9.81-\mathrm{a}^{*} \mathrm{x}(\mathrm{i}, 2)^{\wedge} 2 * \sin (\mathrm{x}(\mathrm{i}, 1))-\mathrm{b}^{*}\right.$ thetadd(i)* $\left.\cos (\mathrm{x}(\mathrm{i}, 1))\right)$;

$\mathrm{m}^{*}\left(1+\mathrm{a}^{*} \cos (\mathrm{x}(\mathrm{i}, 1))\right)^{*}\left(9.81-\mathrm{a}^{*} \mathrm{x}(\mathrm{i}, 2)^{\wedge} 2^{*} \sin (\mathrm{x}(\mathrm{i}, 1)) \ldots\right.$

- $b^{*}$ thetadd $\left.(\mathrm{i}) * \cos (x(\mathrm{i}, 1))\right)+\mathrm{m}^{*}(\mathrm{~h}+\mathrm{b} * \sin (\mathrm{x}(\mathrm{i}, 1))) *\left(\mathrm{~b} * \mathrm{x}(\mathrm{i}, 2)^{\wedge} 2 * \cos (\mathrm{x}(\mathrm{i}, 1)) \ldots\right.$

- a*thetadd(i)*sin(x(i,1)))];

$X=A \backslash B$ 


$$
\begin{aligned}
& \mathrm{D}(\mathrm{i})=\mathrm{X}(2)+\mathrm{X}(3) \\
& \text { output }(\mathrm{i}, 1)=\mathrm{X}(1) \\
& \text { output }(\mathrm{i}, 2)=\mathrm{X}(2) \\
& \text { output }(\mathrm{i}, 3)=\mathrm{X}(3) \\
& \text { output }(\mathrm{i}, 4)=\mathrm{D}(\mathrm{i}) \\
& \text { end }
\end{aligned}
$$

\section{figure;}

hold on; plot(t,output(:,2),'r');

plot(t,output(:,3),'b');

ylabel('Reaction [N] in the y-direction');

xlabel('Time');

grid;

figure;

plot(t,output(:,1), 'black'); ylabel('Reaction [N] at R1x');

xlabel('Time');

grid;
\%Reaction at R1x

$\%$ Reaction at R1y

\%Reaction at R2y 
$\% * * * * * * * * * * * * * * * * * * * * * * * * * * * * * * * * * * * * * * * * * * * * * * * * * * * * * * * * * * * * * * * * * * * *$

$\%$ This Subprogram will find the Angular Displacement, Angular Velocity, and Angular $\%$ Acceleration of both Theta and Fi

$\%$ This is used when we have lift from the left side, when $\mathrm{R}_{1 \mathrm{y}}$ is negative

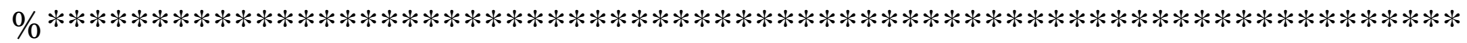

function ydot $=$ pend $(\mathrm{t}, \mathrm{Y})$

global a b acc_ratio t_start

$1=0.45 ; \quad \mathrm{h}=0.6095 ; \quad \mathrm{g}=9.81$

if $\mathrm{t}>=\mathrm{t} \_$start

$\mathrm{xdd}=$ acc_ratio $* 9.81$;

else

xdd $=0$;

end

$\mathrm{c}=\left(\mathrm{a}^{*} \sin (\mathrm{Y}(1))\right)^{\wedge} 2+\left(\mathrm{b}^{*} \cos (\mathrm{Y}(1))\right)^{\wedge} 2$;

$\%$ Theta Double Dot

$\mathrm{d}=0.5^{*}\left(\mathrm{a}^{\wedge} 2-\mathrm{b}^{\wedge} 2\right)^{*} \sin \left(2^{*} \mathrm{Y}(1)\right)$

$\%$ Theta Dot Square

$\mathrm{e}=\mathrm{a}^{*} \mathrm{~h}^{*} \sin (\mathrm{Y}(1))-\mathrm{b}^{*} \mathrm{l}^{*} \cos (\mathrm{Y}(1))+\mathrm{a}^{*} \mathrm{~b}$

$\mathrm{f}=\mathrm{a}^{*} \mathrm{l}^{*} \sin (\mathrm{Y}(1))-\mathrm{b} * \mathrm{~h} * \cos (\mathrm{Y}(1))-0.5^{*}\left(\mathrm{a}^{\wedge} 2+\mathrm{b}^{\wedge} 2\right) * \sin (2 * \mathrm{Y}(1))$;

$\%$ Phi Double Dot

\& Theta Double dot

$\mathrm{n}=\mathrm{g} *\left(\mathrm{~b} * \cos (\mathrm{Y}(1)) * \cos (\mathrm{Y}(3))-\mathrm{a}^{*} \sin (\mathrm{Y}(1)) * \sin (\mathrm{Y}(3))\right)$;

$\%$ Phi Dot Square

$\mathrm{o}=\mathrm{xdd} *\left(\mathrm{a} * \sin (\mathrm{Y}(1)) * \cos (\mathrm{Y}(3))+\mathrm{b}^{*} \cos (\mathrm{Y}(1)) * \sin (\mathrm{Y}(3))\right)$;

$\mathrm{p}=\mathrm{a} * \mathrm{~h} * \cos (\mathrm{Y}(1))+\mathrm{b}^{*} \mathrm{l}^{*} \sin (\mathrm{Y}(1))$

$\%$ Theta Dot Square

$\mathrm{q}=\left(1-\mathrm{a}^{*} \cos (\mathrm{Y}(1))\right)^{\wedge} 2+\left(\mathrm{h}+\mathrm{b}^{*} \sin (\mathrm{Y}(1))\right)^{\wedge} 2$;

$\%$ Phi Double Dot

$\mathrm{v}=-2 * \mathrm{a}^{*} \mathrm{l}^{*} \sin (\mathrm{Y}(1))+2 * \mathrm{~b}^{*} \mathrm{~h} * \cos (\mathrm{Y}(1))+\left(\mathrm{a}^{\wedge} 2+\mathrm{b}^{\wedge} 2\right)^{*} \sin \left(2^{*} \mathrm{Y}(1)\right) ; \%$ Theta Dot $*$ Phi Dot

$\mathrm{r}=-\mathrm{g} *\left(\left(1-\mathrm{a}^{*} \cos (\mathrm{Y}(1))\right) * \cos (\mathrm{Y}(3))+\left(\mathrm{h}+\mathrm{b}^{*} \sin (\mathrm{Y}(1))\right)^{*} \sin (\mathrm{Y}(3))\right)$;

$\mathrm{s}=-\mathrm{xdd} *\left((1-\mathrm{a} * \cos (\mathrm{Y}(1))) * \sin (\mathrm{Y}(3))-\left(\mathrm{h}+\mathrm{b}^{*} \sin (\mathrm{Y}(1))\right) * \cos (\mathrm{Y}(3))\right)$;

$\operatorname{ydot}(1,1)=\mathrm{Y}(2)$

$\operatorname{ydot}(2,1)=\left(\left(d^{*} \mathrm{q}-\mathrm{e}^{*} \mathrm{p}\right)^{*} \mathrm{Y}(2)^{\wedge} 2+\mathrm{f}^{*} \mathrm{q}^{*} \mathrm{Y}(4)^{\wedge} 2+\mathrm{q}^{*}(\mathrm{o}+\mathrm{n})-\mathrm{e}^{*} \mathrm{v}^{*} \mathrm{Y}(2)^{*} \mathrm{Y}(4)+\ldots\right.$ $\left.\mathrm{e}^{*}(\mathrm{r}+\mathrm{s})\right) /\left(\mathrm{e}^{\wedge} 2-\mathrm{c}^{*} \mathrm{q}\right)$;

$\mathrm{ydot}(3,1)=\mathrm{Y}(4)$

$\operatorname{ydot}(4,1)=\left(\left(d^{*} \mathrm{e}-\mathrm{c}^{*} \mathrm{q}\right) * \mathrm{Y}(2)^{\wedge} 2+\mathrm{e}^{*} \mathrm{f}^{*} \mathrm{Y}(4)^{\wedge} 2+\mathrm{e}^{*}(\mathrm{o}+\mathrm{n})-\mathrm{c}^{*} \mathrm{v}^{*} \mathrm{Y}(2)^{*} \mathrm{Y}(4)+\ldots\right.$ $\left.\mathrm{c}^{*}(\mathrm{r}+\mathrm{s})\right) /\left(\mathrm{c}^{*} \mathrm{q}-\mathrm{e}^{\wedge} 2\right)$; 
$\%$ This Program uses the Subprogram above to print the results for Theta and Phi, $\%$ It is only good when $\mathrm{Fi}$ is in the fourth quadrant $(-90<\phi<0)$.

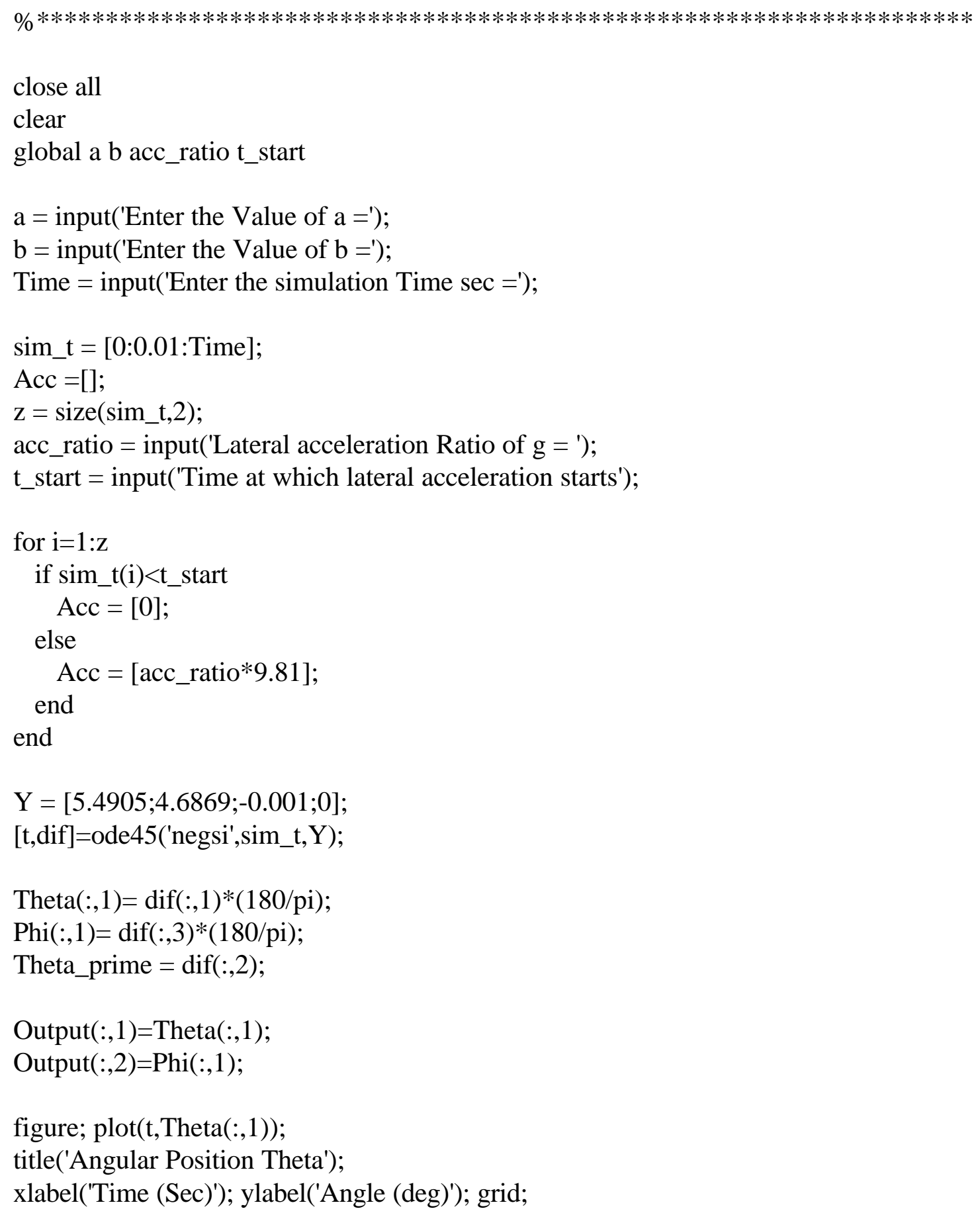


figure; $\operatorname{plot}(\mathrm{t}, \operatorname{dif}(:, 2))$;

title('Angular Velocity Theta-prime');

xlabel('Time (Sec)'); ylabel('Angle Velocity (rad/sec)'); grid;

figure; $\operatorname{plot}(\mathrm{t}, \mathrm{Phi}(:, 1))$;

title('Angular Position Fi');

xlabel('Time (Sec)'); ylabel('Angle (deg)'); grid;

figure; $\operatorname{plot}(t, \operatorname{dif}(:, 4))$;

title('Angular Velocity Fi-prime');

xlabel('Time (Sec)'); ylabel('Angle Velocity (rad/sec)'); grid;

for $\mathrm{i}=1: \mathrm{z}$

fprintf('InTheta $=\% \mathrm{f} \quad$ Phi $=\% \mathrm{f} \quad \mathrm{i}=\% \mathrm{~d}$ ',Output(i,1),Output(i,2),i);

end

for $\mathrm{i}=2: \mathrm{z}-1$

$\mathrm{dt}=\mathrm{t}(2)-\mathrm{t}(1)$;

Theta_2prime(i) $=(\operatorname{dif}(\mathrm{i}+1,2)-\operatorname{dif}(\mathrm{i}-1,2)) /(2 * \mathrm{dt})$;

Phi_2prime $(\mathrm{i})=(\operatorname{dif}(\mathrm{i}+1,4)-\operatorname{dif}(\mathrm{i}-1,4)) /(2 * \mathrm{dt})$;

end

Theta_2prime $(\mathrm{z})=0$;

Phi_2prime $(\mathrm{z})=0$;

figure; plot(t,Theta_2prime);

title('Angular Acceleration Theta-2prime');

xlabel('Time (sec)'); ylabel('Angular Acceleration ( $\left(\mathrm{rad} / \mathrm{sec}^{\wedge} 2\right)$ '); grid;

figure; plot(t,Phi_2prime);

title('Angular Acceleration Phi-2prime');

xlabel('Time (sec)'); ylabel('Angular Acceleration ( $\left.\mathrm{rad} / \mathrm{sec}^{\wedge} 2\right)$ '); grid; 
$\%$ This Subprogram will find the Angular Displacement, Angular Velocity, and Angular $\%$ Acceleration of both Theta and Fi

$\%$ This is used when we have lift from the right side, when $\mathrm{R}_{2 \mathrm{y}}$ is negative

$\% * * * * * * * * * * * * * * * * * * * * * * * * * * * * * * * * * * * * * * * * * * * * * * * * * * * * * * * * * * * * * * * * * * * *$

function $y d o t=$ pend $(t, Y)$

global a b acc_ratio t_start

$1=0.45 ; \quad \mathrm{h}=0.6095 ; \quad \mathrm{g}=9.81$

if $\mathrm{t}>=\mathrm{t} \_$start

xdd=acc_ratio*9.81;

else

$\mathrm{xdd}=0$;

end

$\mathrm{c}=\left(\mathrm{a}^{*} \sin (\mathrm{Y}(1))\right)^{\wedge} 2+\left(\mathrm{b}^{*} \cos (\mathrm{Y}(1))\right)^{\wedge} 2 ; \quad$ \% Theta Double Dot

$\mathrm{d}=0.5^{*}\left(\mathrm{a}^{\wedge} 2-\mathrm{b}^{\wedge} 2\right)^{*} \sin (2 * \mathrm{Y}(1)) ; \quad \%$ Theta Dot Square

$\mathrm{e}=\mathrm{a}^{*} \mathrm{~h}^{*} \sin (\mathrm{Y}(1))+\mathrm{b}^{*} \mathrm{l}^{*} \cos (\mathrm{Y}(1))+\mathrm{a} * \mathrm{~b} ; \quad \%$ Phi Double Dot \& Theta Dot $*$ Phi Dot

$\mathrm{f}=-\mathrm{a}^{*} \mathrm{l}^{*} \sin (\mathrm{Y}(1))+\mathrm{b}^{*} \mathrm{~h}^{*} \cos (\mathrm{Y}(1))+0.5^{*}\left(\mathrm{~b}^{\wedge} 2-\mathrm{a}^{\wedge} 2\right)^{*} \sin \left(2^{*} \mathrm{Y}(1)\right) ; \quad \%$ Phi Dot Square

$\mathrm{n}=\mathrm{g} *\left(\mathrm{~b} * \cos (\mathrm{Y}(1)) * \cos (\mathrm{Y}(3))-\mathrm{a}^{*} \sin (\mathrm{Y}(1))^{*} \sin (\mathrm{Y}(3))\right)$

$\mathrm{o}=\mathrm{xdd} *\left(\mathrm{a} * \sin (\mathrm{Y}(1)) * \cos (\mathrm{Y}(3))+\mathrm{b}^{*} \cos (\mathrm{Y}(1)) * \sin (\mathrm{Y}(3))\right)$;

$\mathrm{p}=\mathrm{a} * \mathrm{~h} * \cos (\mathrm{Y}(1))-\mathrm{b} * \mathrm{l}^{*} \sin (\mathrm{Y}(1))$

$\mathrm{q}=\left(1+\mathrm{a}^{*} \cos (\mathrm{Y}(1))\right)^{\wedge} 2+\left(\mathrm{h}+\mathrm{b}^{*} \sin (\mathrm{Y}(1))\right)^{\wedge} 2$

\% Theta Double Dot

$\mathrm{r}=\mathrm{g} *\left((1+\mathrm{a} * \cos (\mathrm{Y}(1))) * \cos (\mathrm{Y}(3))-\left(\mathrm{h}+\mathrm{b}^{*} \sin (\mathrm{Y}(1))\right) * \sin (\mathrm{Y}(3))\right)$;

$\%$ Phi Double Dot

$\mathrm{s}=\mathrm{xdd} *\left(\left(1+\mathrm{a}^{*} \cos (\mathrm{Y}(1))\right) * \sin (\mathrm{Y}(3))+\left(\mathrm{h}+\mathrm{b}^{*} \sin (\mathrm{Y}(1))\right) * \cos (\mathrm{Y}(3))\right)$;

$\operatorname{ydot}(1,1)=\mathrm{Y}(2)$;

$\operatorname{ydot}(2,1)=\left(\left(d^{*} \mathrm{q}-\mathrm{e}^{*} \mathrm{p}\right)^{*} \mathrm{Y}(2)^{\wedge} 2-\mathrm{f}^{*} \mathrm{q}^{*} \mathrm{Y}(4)^{\wedge} 2+\mathrm{q}^{*}(\mathrm{n}-\mathrm{o})-2^{*} \mathrm{e}^{*} \mathrm{f}^{*} \mathrm{Y}(2)^{*} \mathrm{Y}(4)+\ldots\right.$ $\left.\mathrm{e}^{*}(\mathrm{~s}-\mathrm{r})\right) /\left(\mathrm{e}^{\wedge} 2-\mathrm{c}^{*} \mathrm{q}\right)$

$\operatorname{ydot}(3,1)=\mathrm{Y}(4)$;

$\operatorname{ydot}(4,1)=\left(\left(c^{*} p-d^{*} e\right) Y(2)^{\wedge} 2-e^{*} f^{*} Y(4)^{\wedge} 2+e^{*}(n-o)-2 * c^{*} f^{*} Y(2)^{*} \mathrm{Y}(4)+\ldots\right.$ $\left.\mathrm{c}^{*}(\mathrm{~s}-\mathrm{r})\right) /\left(\mathrm{c}^{*} \mathrm{q}-\mathrm{e}^{\wedge} 2\right)$ 


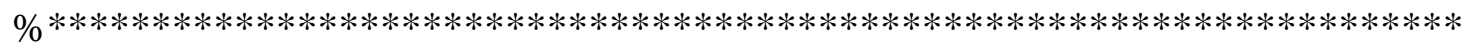

$\%$ This Program uses the Subprogram above to print the results for Theta and Phi, $\%$ It is only good when Phi is in the first quadrant $(0<\phi<90)$.

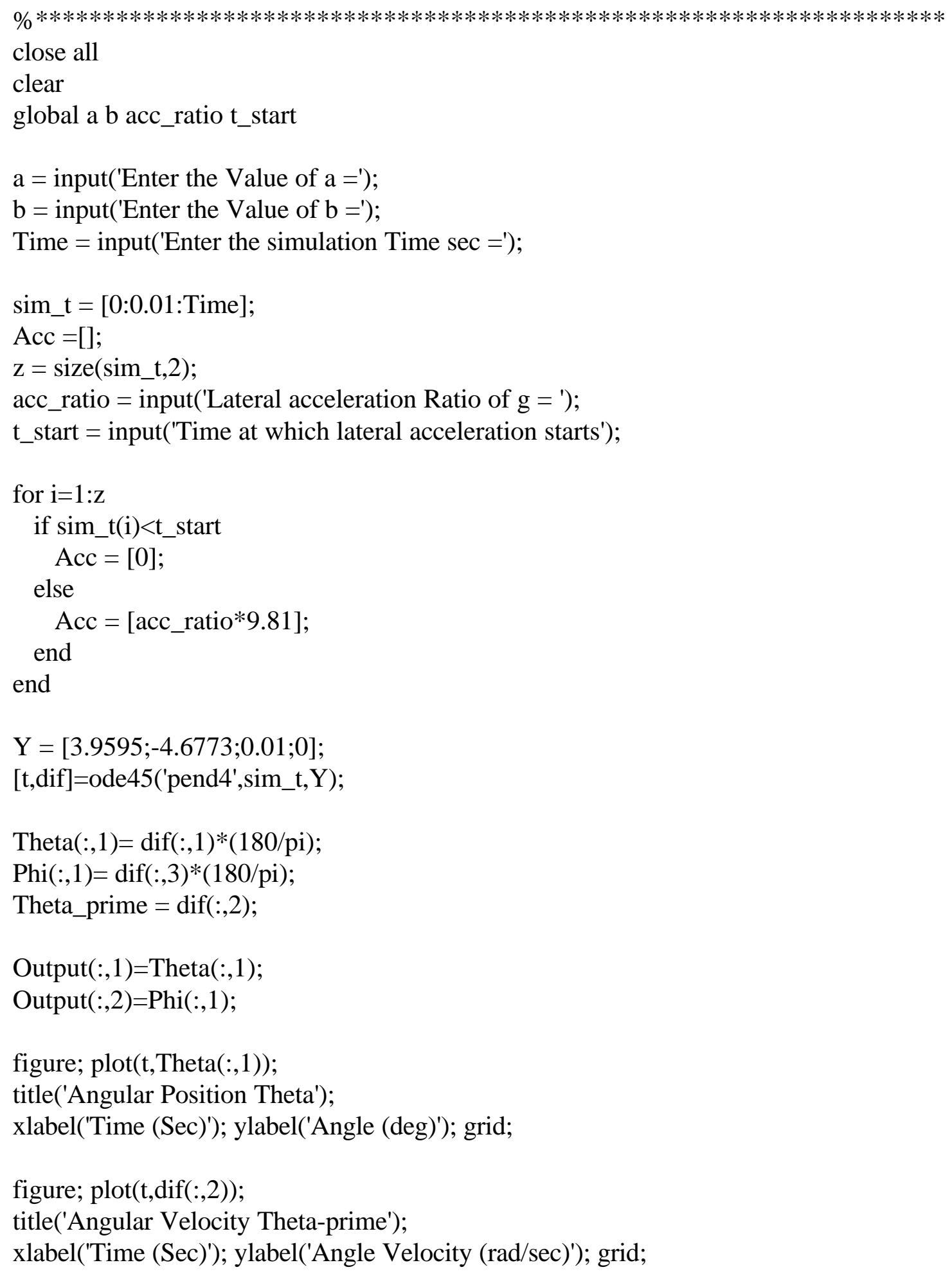


figure; $\operatorname{plot}(\mathrm{t}, \operatorname{Phi}(:, 1))$;

title('Angular Position Fi');

xlabel('Time (Sec)'); ylabel('Angle (deg)'); grid;

figure; $\operatorname{plot}(\mathrm{t}, \operatorname{dif}(:, 4))$;

title('Angular Velocity Fi-prime');

xlabel('Time (Sec)'); ylabel('Angle Velocity (rad/sec)'); grid;

for $\mathrm{i}=1: \mathrm{z}$

fprintf('InTheta $=\% \mathrm{f} \quad$ Phi $=\% \mathrm{f} \quad \mathrm{i}=\% \mathrm{~d}$ ',Output(i,1),Output(i,2),i);

end

for $\mathrm{i}=2: \mathrm{z}-1$

$\mathrm{dt}=\mathrm{t}(2)-\mathrm{t}(1)$;

Theta_2prime $(\mathrm{i})=(\operatorname{dif}(\mathrm{i}+1,2)-\operatorname{dif}(\mathrm{i}-1,2)) /(2 * \mathrm{dt})$;

Phi_2prime $(\mathrm{i})=(\operatorname{dif}(\mathrm{i}+1,4)-\operatorname{dif}(\mathrm{i}-1,4)) /(2 * \mathrm{dt})$;

end

Theta_2prime $(\mathrm{z})=0$;

Phi_2prime $(\mathrm{z})=0$;

figure; plot(t,Theta_2prime);

title('Angular Acceleration Theta-2prime');

xlabel('Time (sec)'); ylabel('Angular Acceleration ( $\left.\mathrm{rad} / \mathrm{sec}^{\wedge} 2\right)$ '); grid;

figure; plot(t,Phi_2prime);

title('Angular Acceleration Phi-2prime');

xlabel('Time (sec)'); ylabel('Angular Acceleration ( $\left.\mathrm{rad} / \mathrm{sec}^{\wedge} 2\right)$ '); grid; 